\title{
DYNAMIC FINITE ELEMENT MODELLING AND FREE VIBRATION ANALYSIS OF TWO DIMENSIONAL ELEMENTS
}

\author{
By: \\ Mohammad Moeid Elahikahooker \\ Bachelor of Engineering, Institute of Space Technology, Islamabad, Pakistan (2014)
}

\author{
A thesis \\ presented to Ryerson University \\ in partial fulfillment of the \\ requirements for the degree of \\ Master of Applied Science \\ in the program of \\ Aerospace Engineering
}

Toronto, Ontario, Canada, 2017

CMohammad Moeid Elahikahooker 


\section{AUTHOR'S DECLARATION FOR ELECTRONIC SUBMISSION OF A THESIS}

I hereby declare that I am the sole author of this thesis. This is a true copy of the thesis, including any required final revisions, as accepted by my examiners.

I authorize Ryerson University to lend this thesis to other institutions or individuals for the purpose of scholarly research.

I further authorize Ryerson University to reproduce this thesis by photocopying or by other means, in total or in part, at the request of other institutions or individuals for the purpose of scholarly research.

I understand that my thesis may be made electronically available to the public. 


\section{ABSTRACT}

Dynamic Finite Element Modelling and Free Vibration Analysis of Two Dimensional Elements

Mohammad Moeid Elahikahooker

Master of Applied Science, Aerospace Engineering, Ryerson University, Toronto (2017)

DFE is proven to be a powerful tool for analysis of structural problems involving vibration of beams, by introducing frequency dependence in element matrices. This method is extended here to thin plate vibration cases. Lack of previous systematic approach and great performance of beam DFEs were particular drives of the present work. Kirchhoff's plate bending theory is derived and used as the reference equation describing element behaviour. DFE is implemented in MATLAB ${ }^{\circledR}$ software and evaluated for different conditions. FEM plates are developed for similar geometry using MATLAB ${ }^{\circledR}$ as well and their performance is compared against DFE formulation. Convergence studies are performed and comparisons between FEM and DFE elements are shown. Moreover, critical review of previous works on plate vibrational modelling is made which highlighted the difficulty involved in solving the partial differential equation of plates. Finally comments about the current element and the potential direction of future research are given. 


\section{ACKNOWLEDGEMENTS}

I would like to thank my family especially my parents for supporting me through different stages of life and motivating me in hard circumstances.

I would also like to thank Prof. Seyed M. Hashemi for his unending patience, wisdom, and guidance throughout my graduate studies at Ryerson, different projects and finally my research, as well as his encouragement and understanding when I needed them. 


\section{TABLE OF CONTENTS}

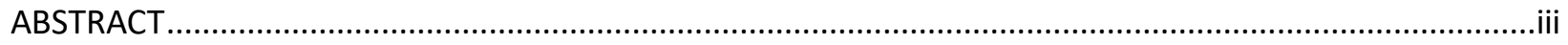

LIST OF TABLES

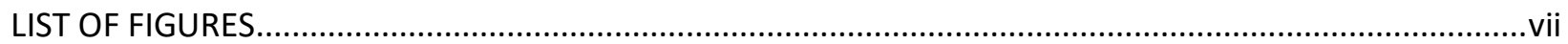

NOMENCLATURE

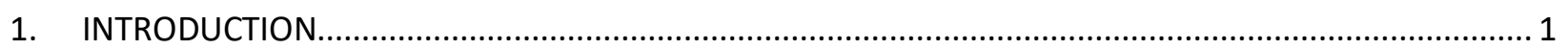

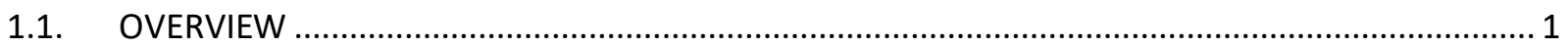

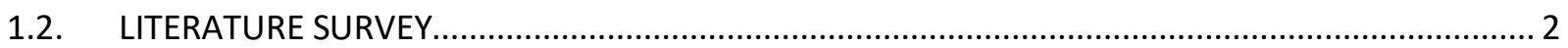

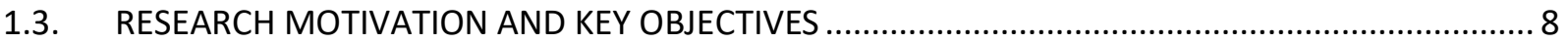

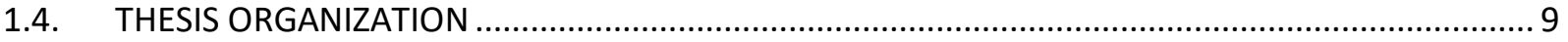

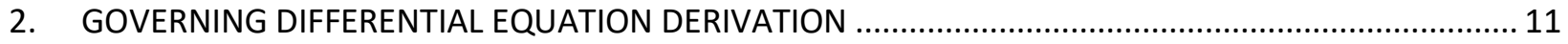

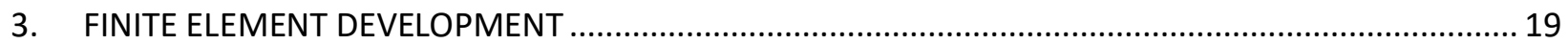

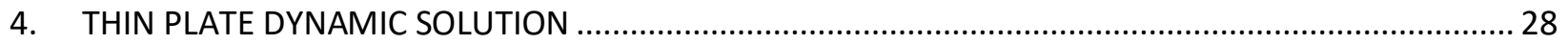

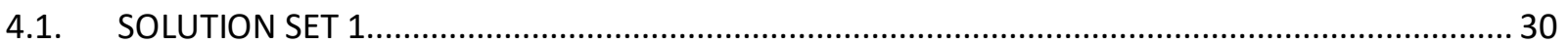

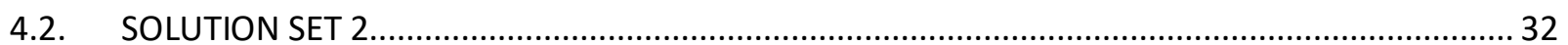

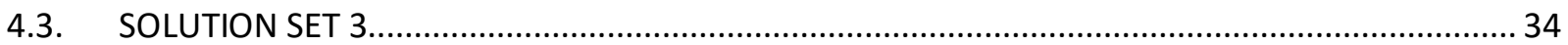

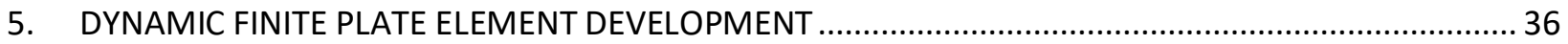

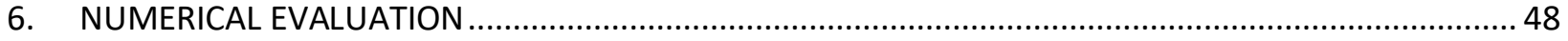

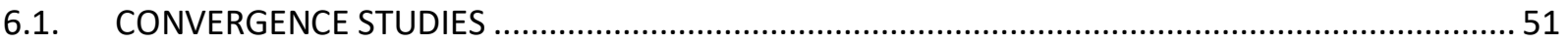

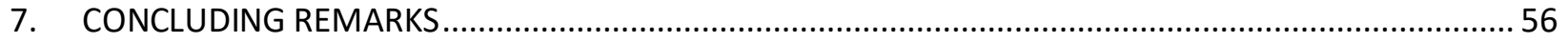

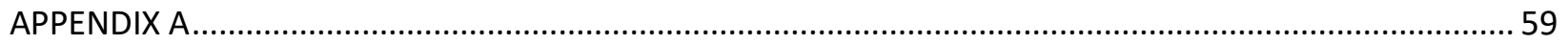

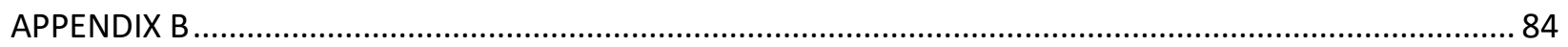

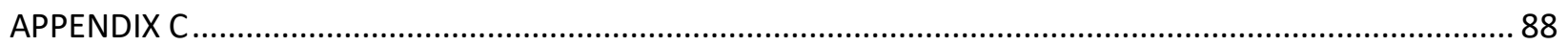

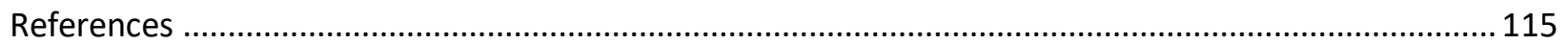




\section{LIST OF TABLES}

Table 1 Comparison of DFE formulation and analytical solution for Values of $\omega \rho h / D$......................... 50

Table 2 Comparison of fundamental natural frequency obtained from DFE and FEM element for Values

of $\omega \rho h / D$

Table 3 Second mode convergence analysis of DFE and FEM elements for $\omega \rho h / D$ on a SS-SS-SS-F

plate.... 53

Table 4 Third mode convergence analysis of DFE and FEM elements for $\omega \rho h / D$ on a SS-SS-SS-F plate

Table 5 Fourth mode convergence analysis of DFE and FEM elements for $\omega \rho h / D$ on a SS-SS-SS-F plate

Table 6 Fifth mode convergence analysis of DFE and FEM elements for $\omega \rho h / D$ on a SS-SS-SS-F plate 


\section{LIST OF FIGURES}

Figure 1 General plate element (a) and differential segment for analysis (b) .......................................... 11

Figure 2 Distribution of external loads (Left) and corresponding internal stress (right) over a infinitesimal

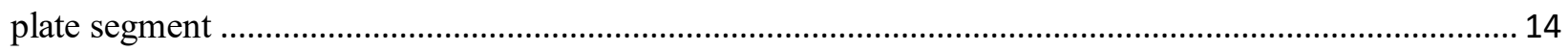

Figure 3 Plate element used for development of finite element ............................................................ 21

Figure 4 Plate element used for development of DFE............................................................................ 36

Figure 5 Graph of DFE shape function $N 1, \omega$ for value of $\gamma x y=10 e-6$ (a) and for value of $\gamma x y=2$

(b) and first Hermite shape function (c) over the plate element of Figure 4........................................... 45

Figure 6 Square plate with two unit side length under SS-SS-SS-F boundary condition ......................... 49

Figure 7 Plot of transcendental DFE determinant function for SS-SS-SS-F boundary condition against $\gamma x y=\rho h \omega 2 D 14$

Figure 8 Comparison of convergence of $\omega \rho h / D$ values between FEM and DFE models for second mode of vibration of a plate under SS-SS-SS-F boundary condition................................................................ 53

Figure 9 Comparison of convergence of $\omega \rho h / D$ values between FEM and DFE models for third mode of vibration of a plate under SS-SS-SS-F boundary condition ................................................................... 54 Figure 10 Comparison of convergence of $\omega \rho h / D$ values between FEM and DFE models for fourth mode of vibration of a plate under SS-SS-SS-F boundary condition................................................................. 54 Figure 11 Comparison of convergence of $\omega \rho h / D$ values between FEM and DFE models for fifth mode of vibration of a plate under SS-SS-SS-F boundary condition ................................................................... 55 Figure 12 Graph of DFE shape function $N 1, \omega$ for value of $\gamma x y=10 e-6$ (a) and for value of $\gamma x y=2$ (b) and first Hermite shape function (c) over the plate element of Figure 3 ........................................... 61 Figure 13 Graph of DFE shape function derivative $\partial N 2, \omega \partial x$ for value of $\gamma x y=10 e-6$ (a) and for value of $\gamma x y=2$ (b) and second Hermite shape function derivative with respect to $x$ (c) over the plate element of Figure 3.

Figure 14 Graph of DFE shape function derivative $\partial N 3, \omega \partial y$ for value of $\gamma x y=10 e-6$ (a) and for value of $\gamma x y=2$ (b) and third Hermite shape function derivative with respect to $x$ (c) over the plate element of Figure 3 .

Figure 15 Graph of DFE shape function $N 4, \omega$ for value of $\gamma x y=10 e-6$ (a) and for value of $\gamma x y=2$ (b) and fourth Hermite shape function (c) over the plate element of Figure 3

Figure 16 Graph of DFE shape function derivative $\partial N 5, \omega \partial x$ for value of $\gamma x y=10 e-6$ (a) and for value of $\gamma x y=2$ (b) and fifth Hermite shape function derivative with respect to $x$ (c) over the plate element of Figure 3.

Figure 17 Graph of DFE shape function derivative $\partial N 6, \omega \partial y$ for value of $\gamma x y=10 e-6$ (a) and for value of $\gamma x y=2$ (b) and sixth Hermite shape function derivative with respect to $y$ (c) over the plate

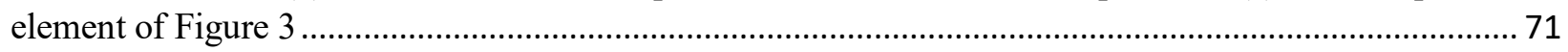
Figure 18 Graph of DFE shape function $N 7, \omega$ for value of $\gamma x y=10 e-6$ (a) and for value of $\gamma x y=2$ (b) and seventh Hermite shape function (c) over the plate element of Figure 3.

Figure 19 Graph of DFE shape function derivative $\partial N 8, \omega \partial x$ for value of $\gamma x y=10 e-6$ (a) and for value of $\gamma x y=2(\mathrm{~b})$ and eighth Hermite shape function derivative with respect to $x$ (c) over the plate element of Figure 3.

Figure 20 Graph of DFE shape function derivative $\partial N 9, \omega \partial y$ for value of $\gamma x y=10 e-6$ (a) and for value of $\gamma x y=2$ (b) and ninth Hermite shape function derivative with respect to $y(c)$ over the plate element of Figure 3.

Figure 21 Graph of DFE shape function $N 10, \omega$ for value of $\gamma x y=10 e-6$ (a) and for value of $\gamma x y=$ 2 (b) and tenth Hermite shape function (c) over the plate element of Figure 3. 79 
Figure 22 Graph of DFE shape function derivative $\partial N 11, \omega \partial x$ for value of $\gamma x y=10 e-6$ (a) and for value of $\gamma x y=2(\mathrm{~b})$ and eleventh Hermite shape function derivative with respect to $x$ (c) over the plate element of Figure 3.

Figure 23 Graph of DFE shape function derivative $\partial N 12, \omega \partial y$ for value of $\gamma x y=10 e-6$ (a) and for value of $\gamma x y=2$ (b) and eleventh Hermite shape function derivative with respect to $y$ (c) over the plate element of Figure 3.

Figure 24 Natural coordinate system (a) is used to map arbitrary shapes from element coordinate system (b) on a 2 by 2 square. 
NOMENCLATURE

\begin{tabular}{|c|c|}
\hline$\varepsilon$ & Axial strain \\
\hline$L$ & Length of differential segment \\
\hline$r$ & Radius of curvature \\
\hline$\rho$ & Density \\
\hline$\theta$ & Deformation angle from curvature center \\
\hline$u$ & Displacement in $x$ direction \\
\hline$v$ & Displacement in $y$ direction \\
\hline$\sigma$ & Axial stress \\
\hline$E$ & Elastic modulus \\
\hline$v$ & Poisson's ratio \\
\hline$\gamma$ & Shear strain \\
\hline$\tau$ & Shear stress \\
\hline$G$ & Shear modulus \\
\hline$M$ & Moment \\
\hline$F$ & Force \\
\hline$Q$ & Shear flow \\
\hline$h$ & Thickness \\
\hline$D$ & Flexural stiffness \\
\hline$w$ & Flexural displacement \\
\hline$[X]$ & Basis function vector \\
\hline$[a]$ & Constants vector \\
\hline$[P]$ & Basis function/derivative matrix \\
\hline$[N]$ & Shape function vector \\
\hline$\delta$ & Virtual operator \\
\hline$\omega$ & Harmonic vibrational frequency \\
\hline$n$ & Normal vector \\
\hline$d s$ & Differential length segment \\
\hline$[K]$ & Stiffness matrix \\
\hline$\gamma_{x y}$ & Normalized vibrational frequency \\
\hline$[M]$ & Mass matrix \\
\hline
\end{tabular}




\section{INTRODUCTION}

\subsection{OVERVIEW}

Plates are structural elements, commonly used in various industries including civil, automotive and aerospace to name a few. The widespread use of plates is in part due to their ability to resist loads in various directions while having a low weight.

In aerospace industry in particular, plates are used as skin for aircraft and spacecraft fuselage, wings and engine nacelles, to provide a reduced drag envelope for the flying machine. As such, these elements are under tension, compression, bending and twisting of various magnitudes during each flight cycle. Also, presence of rotary gas turbine engine and air turbulence induces fluctuating loads on these components, leading to vibrational response.

Based on loading, plates are analyzed from two different aspects in mechanics. Plates that face in-plane tension/compression are classified as membrane, while the term "plate" is used more specifically, for out-of-plane bending/twisting loads. These two conditions can be modelled

independently. However, in general, plates see both loadings simultaneously, and should be analyzed by superimposing membrane and plate formulations. Such superposition generates shell elements which can be curved to include more geometric configurations.

In this research, the focus is directed towards vibrational analysis of plates under bending/twisting condition. Thus, the use of term plate will refer to such loadings, and does not include membrane behaviour.

Vibration is characterized by rapidly changing internal stress directions and magnitudes. Vibrational response is due to the tendency of objects to return to an equilibrium position. This 
tendency is caused by the stiffness property. Vibration can occur because of disturbance from the equilibrium position, or application of varying external loads.

Vibrations create fatigue in the structural components, reducing their load handling capacity well below yielding point. Also, plate vibration is a major source of cabin noise in both automobiles and aircrafts, causing passenger discomfort. Therefore, it is crucial to have a good understanding and model of study, for the vibrational behaviour of plates, to improve both component life and flight experience.

Mathematical models of plate vibration provide equations that describe the amplitude and phase of vibration, at any given moment in time, on any location within the plate boundaries. These partial differential equations are tedious to solve in full form, and complete analytical solutions are available for only specific geometries and boundary conditions.

To expand the application of these models to various geometries, several solution techniques have been proposed. Generally, these techniques reduce the full solution of the partial differential equation to a simpler form, which results in reduced accuracy.

\subsection{LITERATURE SURVEY}

Due to their versatile use, plates have received research attention for decades. Investigators have tried to develop loading response models that match empirical data and experimental results, in

order to better predict failure, and to provide designers with tools to optimise geometries, and material properties in initial stages of development. In this section, a brief review of plate vibrations, governing theories and models, and solution methods available in literature is presented, and their advantages and disadvantages are highlighted. 
The first mathematical models for flexural plate behaviour were developed by ignoring thickness dependent effects, such as rotary inertia and out of plane shear. Such assumptions are accurate for plates of small thickness. Kirchhoff and Love introduced the governing equation for such plates. For thin plates, therefore, they assumed that the plate thickness will always remain perpendicular to midplane, regardless of the deformation state. Hence, in thin plates, the slope of any line along thickness is equal to the slope of the midplane after deformation. As such, this hypothesis makes thin plates comparable to thin beams, modeled by Euler-Bernoulli model. The governing differential equation of such plates is aptly named, thin plate or Kirchhoff plate model.

Much like beams, for plates of considerable thickness, the rotary inertia and shear effects across plate thickness become large, and invalidate the assumption of thickness perpendicularity, proposed by thin plate model. To account for these effects, the thick plate model was proposed by Reissner [1]- [2] and Mindlin [3]. This model couples the rotation along the thickness with midplane slope, without forcing equality, thereby correcting the thickness assumption of the thin plate model.

These two theories provide governing equations, which describe the transverse behaviour of plates under flexural loading. These governing equations are in form of two-dimensional partial differential equations in planar coordinates. To find the solution of these equations, various methods have been developed with advantages and disadvantages of their own.

Analytical methods strive to determine the closed form solution of these governing partial differential equations, when applied to a plate of known domain. Since plate equations are partial differential equations, their analytical solution involves infinite number of functions. Therefore, these complete analytical solutions are very complex and have been developed for specific problems only. This complexity reduces their attraction as a tool for analysis. 
Navier method is a well-known technique for solving such differential equations. It formulates the solution as a double Fourier series with unknown constants. These constants are found parametrically such that, the solution satisfies plate's kinematics, dynamic and boundary conditions. By application of this method to thin plates, Leissa [4]- [9] evaluated vibrational analysis of various boundary and loading conditions. The Navier method has also been used to analyze vibration and buckling of isotropic and composite plates [10]- [15].

In contrast, the Levy method utilizes a single Fourier series [16] to solve plate equations. Due to the lack of Fourier expansion along one direction, Levy method is applicable to plates with special boundary conditions.

The difficulties imposed by analytical solutions have led to development of approximate solution methods. These methods do not focus on the exact solution of the governing differential equation, rather they propose an approximate solution, whose error can be minimized over the problem domain.

Approximate solutions are obtained by variational formulation, where a weighting function is assigned to the error of the weak form of the governing differential equation. Minimization of the error functional is performed through weighted residual integration. Rayleight [17] and Ritz [18] assume a set of trials for the weighting function while Galerkin's method takes identical weighting and solution functions. These two methods have been used extensively to solve variety of problems for thick and thin plates [19]- [25].

Similar to analytical techniques, approximate methods solve the governing differential equation of plates as a single equation, over the problem domain. This approach requires reformulation for 
every boundary and loading conditions. Also, development of appropriate weighting and basis functions for various element domains can be difficult.

A simpler approach to these problem is use of numerical techniques. In such methods, the problem domain is discretized to a number of smaller geometries, called elements. These elements combine in a mesh structure to generate original domain of the plate.

Arguably, finite element method (FEM) is most widely used numerical technique for static and dynamic analysis of structural components. In FEM analyses, the problem domain is divided into smaller sections called elements. The element is assumed to be characterised by a collection of nodes. Degrees of freedom (DoFs) are representation of the displacements/deformation of the plate on an element location at these nodes.

The development of finite element method is done by application of variational formulations, such as Galerkin or Rayleigh-Ritz technique. However, because the problem domain is subdivided in smaller elements, the weak form solution is applied to a small section of the domain, and error is reduced by making the sections smaller. Hence, the assumed solution and weighting functions, do not need to be solutions of the governing differential equation. For example, many FEM formulations have used polynomials of various degrees as their solution and weight functions. Using polynomials leads to further ease in numerical evaluation.

Simplicity in development and applicability has led to widespread use of finite element formulation. A rich body of literature is available on FEM analysis of plates, and their boundedness and convergence properties are well understood. The FEM elements are easily extended to plates of arbitrary geometries, such as quadrilaterals and curved edge elements with good convergence [26]. FEM has been used for variety of difficult loadings and configurations, 
such as plates having cut-outs, laminated composite plates, thick composite plates and large amplitude bending [27]- [37].

The simplicity and good convergence of the FEM formulation is also exploited by many engineering analysis software. The main reason for this trend is that the FEM vibrational analysis results in linear Eigenvalue problems. These linear Eigenvalue problems are relatively straight forward to solve and numerous mathematical and computer algorithms have been developed for fast and precise evaluation. Ansys Inc. and MSC software Inc. are example of such software.

The desire to develop elements with wide applicability of FEM and accuracy of exact models has given rise to hybrid techniques. Dynamic Stiffness Matrix (DSM) and Dynamic Finite Element (DFE) are among the well-stablished hybrid methods. Both methods are developed for beam problems and recent extension to plates are being researched. A summary of these hybrid solution techniques is outlined here.

DSM involves a complete solution of the beam equation for simple harmonic oscillation cases. By using D'Alembert's principle, researchers include the dynamic mass term, as part of the governing differential equation. The resulting ordinary differential equation is solved, generating frequency dependent hyperbolic and trigonometric solutions.

These solutions are organized in a displacement matrix. This displacement matrix is then related to a force matrix, using a differential operator matrix. The operator matrix is derived based on load-stress and stress-deformation relationships of thick/thin models. Application of the boundary and loading condition, shapes the final dynamic stiffness matrix for the entire problem domain. 
This frequency dependent matrix is then analyzed for non-linear Eigenvalue problem, which yields a transcendental frequency dependent equation. With the help of William-Wittrick algorithm [38], the determinant equation can be swept across frequency domain to find a frequency value of interest. DSM technique has been mainly applied to beam vibration problems [39]- [44]. . where displacement, force and operator matrix dimensions are finite. In a recent study [45], this method was extended to thin plates.

DFE is another successful alternative to FEM for beam analysis. DFE also organizes the frequency dependent solutions of the governing differential equation in a displacement matrix. However, the stiffness matrix in DFE is not derived for each problem condition.

DFE development procedure is similar to FEM, in that, it follows Galerkin's scheme of development and starts as a weak solution. However, the basis functions used are exact solutions of the governing differential equation. Use of exact solution allows manipulation of Galerkin's method to further simplify the formulation. By applying integration by parts, integrals are reduced and only evaluation of DFE matrices at element boundaries is required.

Another similarity between DFE and FEM, is that it is performed over an element domain. Therefore, the DFE matrices are associated with elements, rather than the entire physical space of the problem. Hence, complex domains can be analyzed by discretizing the problem into regions of similar geometry, material property and loading conditions using a meshing scheme. DFE has been used for analysis of beams with bending-torsion coupling [46], beams with extension-torsion coupling [47], sandwich beams [48], and axially loaded bending-torsion coupled [49] with great success. 
The difference between DFE and FEM is that, DFE produces one frequency dependent stiffness matrix per element while FEM creates frequency independent mass and stiffness matrices. Similar to DSM, the vibrational analysis performed by DFE, consequently involves solving a nonlinear Eigenvalue problem. Because of presence of complete solution of the governing differential equation inside the basis functions, DFE beams show very fast convergence to the analytical solution, making them a viable alternate to conventional FEM.

As a result, much coarser DFE mesh can produce similar results for a problem analyzed with FEM. This coarser mesh can reduce the time required to solve a vibration problem by reducing the time required to mesh the problem domain and reducing the order of the Eigenvalue problem under investigation.

\subsection{RESEARCH MOTIVATION AND KEY OBJECTIVES}

As mentioned in the previous section, hybrid techniques are recent development in vibrational analysis, that have shown good performance and wide applicability for beam problems. These methods can be considered as an intermediate between analytical and numerical solutions. While DSM has been used for plate vibration problems recently, such an expansion has not yet been performed for DFE formulation. The excellent performance of DFE elements in vibrational analysis of beams and a lack of extension of this technique for two dimensional plates served as motivation for this study.

Therefore, the main purpose of the present study is to systematically extend the DFE method to thin Kirchhoff plates. The goal of development of two dimensional DFE here, is to be applicable to plates of arbitrary shapes so that various geometries can be analyzed. The research in DFE 
will ultimately yield a new library of elements with improved dynamic analysis performance which can be used as an alternative to FEM vibrational analysis.

Just like beams, plate DFE requires obtaining the solution of the governing differential equation as the first step. However, because contrary to beam elements, the governing partial differential equation of plates produces an infinite dimensional Hilbert space as its complete solution set, such extension is not as straight forward.

The key objectives of this research are summarized as:

1. Review of plate vibrational analysis models and solution schemes

2. Development of a DFE formulation for plate flexural vibrations

3. Solving governing differential equation for DFE development

4. Evaluation of plate DFE formulation using extracted solutions

5. FEM plate development and comparison with DFE plate

6. Extension of DFE method to arbitrary geometries

\subsection{THESIS ORGANIZATION}

In the following chapters, first a detailed derivation of Kirchhoff plate governing differential equation will be presented to underscore the key assumptions of the thin plate theory and establish relationships between loadings and deformations.

In chapter 3 a review of FEM formulation for plate bending is presented to pave the road for the development of the DFE formulation. FEM formulation for plates have been developed for various number of nodes and DoFs but in this study a four node, 12 DoF FEM plate is developed for direct comparison with DFE plate. The FEM model is based on cubic Hermite functions and 
the use of Galerkin's method of weighted residuals, and yields constant element mass and stiffness matrices.

In chapter 4, DFE plate governing differential equation is solved. The chapter begins with extraction of 3 different solution subsets of interest from the plate equation that satisfy a symmetry and continuity condition. To the author's best knowledge, this study is one of the first attempts on developing 12 DoF DFE formulation for plates based on such solution subsets of the governing differential equation.

Next, in chapter 5, a detailed mathematical derivation of DFE stiffness matrix formulation for plates is presented. The development is based on generation of dynamic matrix integrals from Galerkin's weak from integral of plate equation. The frequency dependent dynamic shape functions are evaluated for use in the DFE integrals and yield the final frequency dependent DFE stiffness matrix.

In chapter 6, The shape functions are placed in the DFE and FEM integrals and 12 DoF plate elements are developed for both methods. The performance of the two technique is compared in this chapter.

In the last chapter concluding remarks about the performance and convergence of DFE plates are given, and finally comments regarding the future research direction and expandability of the formulation are made. 


\section{GOVERNING DIFFERENTIAL EQUATION DERIVATION}

In this chapter, the mathematical formulation of thin plate model is briefly reviewed. A more complete discussion of thin plate theory can be found in structural analysis reference books such as [50].

A thin plate is defined as a geometry, which has two dimensions significantly (at least 10 times) larger than a third dimension, usually called thickness. Thin plate models are used to analyze bending, twisting and out of plane shear loads, while retaining thickness perpendicularity; in other words, the lines along thickness remain parallel to each other and perpendicular to the middle surface of the plate. In this regard, thin plates are two dimensional analogues of EulerBernoulli beams.

The desired goal here, is to relate the deformation of the middle surface of the plate, in flexural direction, to the loading condition it bears. This middle surface deformation will be considered as representative deformation of the entire plate. To start, the internal stresses and strains must be defined in terms of the middle surface displacement $w$.

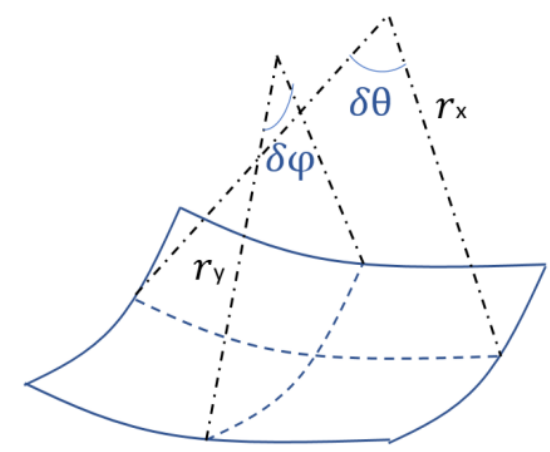

(a)

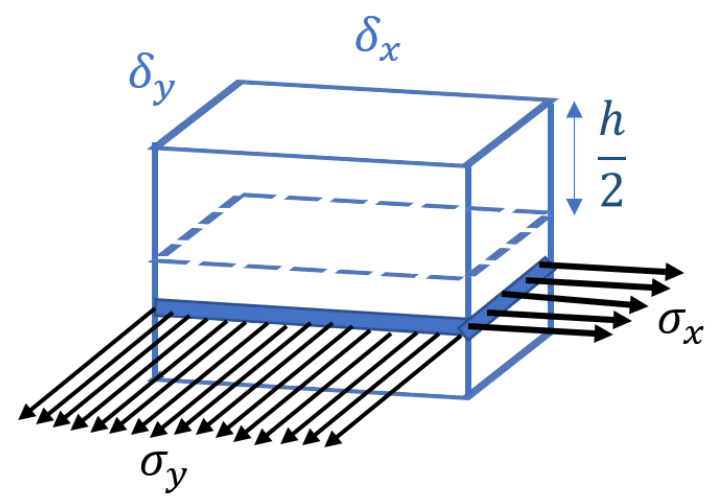

(b)

Figure 1 General plate element (a) and differential segment for analysis (b) 
Consider a layer of a plate at thickness level $z$ below the midplane, which is under application of moments and shear forces. The layer has side lengths $L_{x}$ and $L_{x}^{\prime}$ along $x$ direction, before and after deformation respectively. The axial strain along $x$ axis of this layer $\varepsilon_{x}$, is defined as:

$\varepsilon_{x}=\frac{d u}{d x}=\frac{L_{x}^{\prime}-L_{x}}{L_{x}}=\frac{\left(r_{x}+z\right) \delta \theta-r_{x} \delta \theta}{r_{x} \delta \theta}=\frac{z}{r_{x}}$

Equation 1

Here, $\varepsilon_{x}$ is the axial strain and $u$ denotes axial displacements of the layer in $x$ direction. The term $\delta \theta$ represents bending angle about $y$ axis and $r_{x}$ is the radius of curvature of deformed plate in that direction. For thin plates, applying thickness perpendicularity assumption, geometrically relates this radius to flexural displacement $w$ as:

$\frac{1}{r_{x}}=-\frac{\partial^{2} w}{\partial x^{2}}$

Equation 2

The negative sign is present because the positive direction of radius of curvature is in negative $z$ direction.

Similarly:

$\varepsilon_{y}=\frac{d v}{d x}=\frac{z}{r_{y}}$

Equation 3

$\frac{1}{r_{y}}=-\frac{\partial^{2} w}{\partial x^{2}}$

Equation 4

where, $\varepsilon_{y}$ and $v$ are axial strain and displacement of deformed plate along $y$ direction respectively. $r_{y}$ shows radius of curvature about $x$ axis. 
Axial displacements $u$ and $v$ can now be defined from the above relations in terms of lateral displacement of the middle plane:

$u=-z \frac{\partial w}{\partial x}+c$

Equation 5

$v=-z \frac{\partial w}{\partial y}+c^{\prime}$

Equation 6

The constants $c$ and $c^{\prime}$ account for rigid body displacements. In-plane shear strain is defined as:

$\gamma=\frac{\partial u}{\partial y}+\frac{\partial v}{\partial x}=-2 z \frac{\partial^{2} w}{\partial x \partial y}$

Equation 7

For a thin plate the stresses are planar and therefore, using the constitutive law for planar stress condition, axial stresses can be related to axial strains as:

$\sigma_{x}=\frac{E}{1-v^{2}}\left(\varepsilon_{x}+v \varepsilon_{y}\right)=\frac{E z}{1-v^{2}}\left(\frac{1}{r_{x}}+\frac{v}{r_{y}}\right)$

Equation 8

$\sigma_{y}=\frac{E}{1-v^{2}}\left(\varepsilon_{y}+v \varepsilon_{x}\right)=\frac{E}{1-v^{2}}\left(\frac{1}{r_{y}}+\frac{v}{r_{x}}\right)$

Equation 9

where $\sigma_{x}$ and $\sigma_{y}$ are axial stresses along $x$ and $y$ directions respectively. The term $v$ is Poisson's ratio which relates the effect of axial strain along one axis to axial strain along another axis. $E$ is the elastic modulus of the plate material and is a measure of its stiffness. Both $v$ and $E$ are intrinsic properties of the material.

Stresses can now be related to the flexural displacement $w$ using strain definitions as: 
$\sigma_{x}=\frac{-E z}{1-v^{2}}\left(\frac{\partial^{2} w}{\partial x^{2}}+v \frac{\partial^{2} w}{\partial y^{2}}\right)$

Equation 10

$\sigma_{y}=\frac{-E z}{1-v^{2}}\left(\frac{\partial^{2} w}{\partial y^{2}}+v \frac{\partial^{2} w}{\partial x^{2}}\right)$

Equation 11

$\tau_{x y}=G \gamma=-2 G z \frac{\partial^{2} w}{\partial x \partial y}$

Equation 12

To arrive at the governing differential equation of a thin plate, the relationship between the internal and external loads should be defined under equilibrium. Kirchhoff plates can face moments and forces applied normal to their plane, and in plane loadings are not included in this model. The external loads and internal stresses included in thin plate model, are shown in Figure 2 in their positive directions, for an infinitesimal plate segment of length $\delta x$ and width $\delta y$ and thickness $h$.
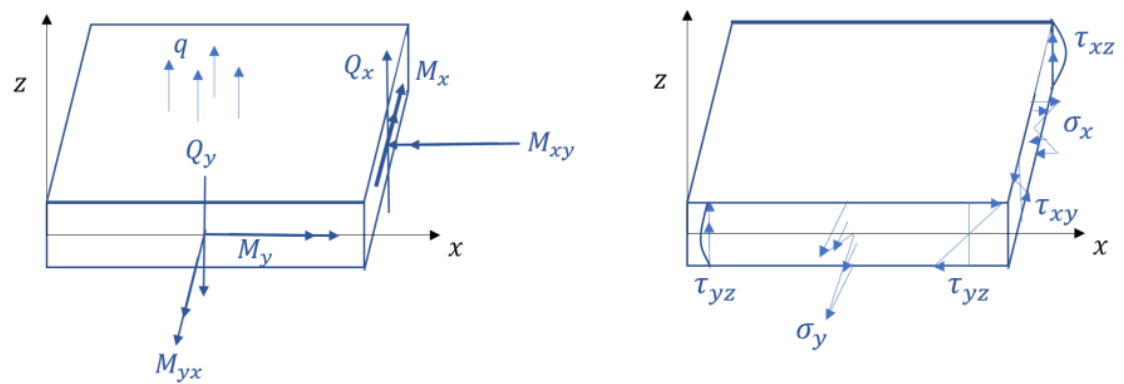

Figure 2 Distribution of external loads (Left) and corresponding internal stress (right) over a infinitesimal plate segment

Starting from moments at boundaries, two bending moments about $x$ and $y$ axis and a twisting moment can be applied to a plate. $M_{y}, M_{x}$ and $M_{x y}$ represent these moments respectively 
normalized per unit length. $M_{x}$ associated moment is balanced by the internal moment created by axial stress $\sigma_{x}$ :

$M_{x} \delta y=\int_{-\frac{h}{2}}^{\frac{h}{2}} \sigma_{x} z \delta y d z=\int_{-\frac{h}{2}}^{\frac{h}{2}}\left(\frac{-E z^{2}}{1-v^{2}}\left(\frac{\partial^{2} w}{\partial x^{2}}+v \frac{\partial^{2} w}{\partial y^{2}}\right)\right) \delta y d z$

Equation 13

$M_{x}=\frac{-E h^{3}}{12\left(1-v^{2}\right)}\left(\frac{\partial^{2} w}{\partial x^{2}}+v \frac{\partial^{2} w}{\partial y^{2}}\right)$

Equation 14

Similarly, $M_{y}$ is counteracted by internal moment created by axial stress $\sigma_{y}$ :

$M_{y}=\frac{-E h^{3}}{12\left(1-v^{2}\right)}\left(\frac{\partial^{2} w}{\partial y^{2}}+v \frac{\partial^{2} w}{\partial x^{2}}\right)$

Equation 15

And the twisting moment of $M_{x y}$ is balanced by internal shear stress $\tau_{x y}$ :

$M_{x y} \delta y=\int_{-\frac{h}{2}}^{\frac{h}{2}} \tau_{x y} z \delta y d z=-\int_{-\frac{h}{2}}^{\frac{h}{2}} 2 G z^{2} \frac{\partial^{2} w}{\partial x \partial y} \delta y d z=-\frac{G h^{3}}{6} \frac{\partial^{2} w}{\partial x \partial y} \delta y$

Equation 16

where $G$ is shear modulus, used in defining the twisting moment. For linearly elastic material the shear modulus is a measure of torsional stiffness, and is related to the elastic modulus by the relation $G=\frac{E}{2(1+v)}=\frac{E}{2\left(1-v^{2}\right)}(1-v)$. Therefore Equation 16 can be rewritten as:

$M_{x y}=-\frac{E h^{3}}{12\left(1-v^{2}\right)}(1-v) \frac{\partial^{2} w}{\partial x \partial y}$

Equation 17

Moving to the $z$ direction forces next, a plate can face shear loads along its sides. The terms $Q_{x}$ and $Q_{y}$ are called shear flows, and are defined as shear force per unit length, applied to a surface 
perpendicular to $x$ and $y$ axis respectively. Application of shear force to plates produces internal shear stresses $\tau_{x z}$ and $\tau_{y z}$ in surfaces normal to $x$ and $y$ axis. Therefore, these shear flow and shear stress terms can be related as:

$Q_{x}=\int_{-\frac{h}{2}}^{\frac{h}{2}} \tau_{x z} d z$

Equation 18

$Q_{y}=\int_{-\frac{h}{2}}^{\frac{h}{2}} \tau_{y z} d z$

Equation 19

Having related the internal stress to external loads, the balance of the external loads can lead to the governing differential equation of the plate in terms of the internal stresses. To do so, first, external forces and moments are balanced under equilibrium, over the infinitesimal plate segment of Figure 2, to achieve the relationship between external loads. The internal stresses can then replace the external loads in this relationship, using the equations previously derived.

Force equilibrium equation in $z$-direction can be written as:

$\sum F_{z}=m a_{z}$

Equation 20

where $F_{z}$ represents any force applied to plate segment in vertical $z$ direction, $m$ is the mass of the infinitesimal segment and $a_{z}$ is the vertical acceleration. By substituting the components of loads contributing to this force summation, following relation is obtained:

$$
\begin{aligned}
& \left(Q_{y}+\frac{\partial Q_{y}}{\partial y} \delta y\right) \delta x-Q_{y} \delta x+\left(Q_{x}+\frac{\partial Q_{x}}{\partial x} \delta x\right) \delta y-Q_{x} \delta y+q \delta x \delta y \\
& =\rho h \delta x \delta y \frac{\partial^{2} w}{\partial t^{2}}
\end{aligned}
$$


where $\rho$ is the volumetric density of the plate material and $q$ is external pressure applied to the plate. Upon simplification Equation 21 can be written as:

$\frac{\partial Q_{y}}{\partial y}+\frac{\partial Q_{x}}{\partial x}+q=\rho h \frac{\partial^{2} w}{\partial t^{2}}$

Equation 22

Similarly, taking equilibrium of moment about positive $x$ axis and performing the balance at center of the plate segment gives:

$-\left(M_{x y}+\frac{\partial M_{x y}}{\partial x} \delta x\right) \delta y+M_{x y} \delta y+\left(Q_{y} \delta x\right) \frac{\delta y}{2}$
$+\left(Q_{y} \delta x+\frac{\partial Q_{y}}{\partial y} \delta y \delta x\right) \frac{\delta y}{2}+M_{y} \delta x-\left(M_{y}+\frac{\partial M_{y}}{\partial y} \delta y\right) \delta x=0$

Equation 23

By balancing moments about $y$ axis next, and considering that for thin plates, the twisting moment is identical in both direction $\left(M_{x y}=M_{y x}\right)$ following relations are obtained after simplification:

$-\frac{\partial M_{x y}}{\partial x}-\frac{\partial M_{y}}{\partial y}+Q_{y}=0$

Equation 24

$-\frac{\partial M_{x y}}{\partial y}-\frac{\partial M_{x}}{\partial x}+Q_{x}=0$

Equation 25

Substituting these two equations in force balance relation (Equation 22) results in:

$\frac{\partial^{2} M_{x}}{\partial x^{2}}+2 \frac{\partial^{2} M_{x y}}{\partial x \partial y}+\frac{\partial^{2} M_{y}}{\partial y^{2}}+q=\rho h \frac{\partial^{2} w}{\partial t^{2}}$

Equation 26

Now, by replacing the external moment terms with their corresponding internal stress relations, the final form of our governing differential equation is reached: 
$-\left(\frac{E t^{3}}{12\left(1-v^{2}\right)}\right)\left(\frac{\partial^{4} w}{\partial x^{4}}+2 \frac{\partial^{4} w}{\partial x^{2} \partial y^{2}}+\frac{\partial^{4} w}{\partial y^{4}}\right)+q=\rho h \frac{\partial^{2} w}{\partial t^{2}}$

Equation 27

For a free vibration analysis, the external applied forces are zero the term $q$ can be eliminated:

$\left(\frac{E t^{3}}{12\left(1-v^{2}\right)}\right)\left(\frac{\partial^{4} w}{\partial x^{4}}+2 \frac{\partial^{4} w}{\partial x^{2} \partial y^{2}}+\frac{\partial^{4} w}{\partial y^{4}}\right)+\rho h \frac{\partial^{2} w}{\partial t^{2}}=0$

Equation 28

For a harmonic vibration, one can assume:

$w(x, y, t)=\bar{w}(x, y) e^{i \omega t}$

Equation 29

where $\bar{w}(x, y)$ represents the amplitude of vibration at any plate location $(x, y)$. This results in:

$D\left(\frac{\partial^{4} \bar{w}}{\partial x^{4}}+2 \frac{\partial^{4} \bar{w}}{\partial x^{2} \partial y^{2}}+\frac{\partial^{4} \bar{w}}{\partial y^{4}}\right)-\omega^{2} \rho h \bar{w}=0$

Equation 30

Here, $D=\left(\frac{E h^{3}}{12\left(1-v^{2}\right)}\right)$ is the flexural stiffness of the plate.

Equation 30 is a homogenous linear partial differential equation which governs the behaviour of a thin plate's lateral displacement, $w$, under harmonic vibrations with no external loadings applied. This model of analysis of plate is known as Kirchhoff plate formulation [50]. For the sake of simplicity, the bar sign on $\bar{w}$ will be dropped in further development and $w$ will represent $\bar{w}$ as vibration amplitude. 


\section{FINITE ELEMENT DEVELOPMENT}

As mentioned previously in the INTRODUCTION chapter, one of the most famous numerical methods used for solving Kirchhoff plate problem is the Finite Element Method (FEM). In FEM analyses, the problem domain is divided into smaller elements. A number of elements are used to cover the entire geometry of the original problem, creating a mesh. Element are assumed to be characterised by a collection of nodes. The element Degrees of Freedom (DoFs) represent the displacements/deformation of the plate at element nodes.

Finite element plates developed for vibrational analysis are an extension to FEM plates used for static problems. For static elements, the governing differential equation developed in last chapter is written as:

$D\left(\frac{\partial^{4} w}{\partial x^{4}}+2 \frac{\partial^{4} w}{\partial x^{2} \partial y^{2}}+\frac{\partial^{4} w}{\partial y^{4}}\right)=q$

Equation 31

While this equation is a partial differential equation in both plane coordinates $x$ and $y$, and generally has infinite number of solutions, FEM formulations use polynomials of finite degrees as their assumed solution functions. This approach provides simplicity in numerical evaluation. The polynomials used by FEM for defining the displacement function satisfy the governing differential equations in static form, and are a subset of complete solution of Equation 31.

Since the displacement polynomial does not include the complete solution, element results are not exact for the plate problems. However, because the problem domain is subdivided in elements, the error is bound to a small section of the domain. By refining the mesh and making the elements smaller, the total error is reduced and the FEM results converge to the analytical solution. 
Adini and Clough [51] used a 12-degree polynomial for development of a four-node plate element with 12 DoFs. This plate element is briefly discussed here for direct comparison with the 12 DoF dynamic finite element developed and presented later in this thesis.

Consider the displacement field to be defined by the 12-term polynomial below:

$$
\begin{aligned}
& w(x, y)=a_{0}+a_{1} x+a_{2} y+a_{3} x^{2}+a_{4} x y+a_{5} y^{2}+a_{6} x^{3}+a_{7} x^{2} y \\
& +a_{8} x y^{2}+a_{9} y^{3}+a_{10} x^{3} y+a_{11} y^{3} x=[X][a]
\end{aligned}
$$

Equation 32

Row vector $[X]$ contains the $X_{i}$ polynomial terms and column vector $[a]$ contains $a_{i}$ constants. These polynomials are called basis functions.

The perpendicularity condition of thin Kirchhoff model, equates the slopes of plates to direct differentials of the displacement fields $w$, in $x$ and $y$ directions. Therefore, by evaluating basis function vector, $[X]$, and its derivative on the element nodal coordinates, a unique relationship can be obtained that relates constant matrix $[a]$ to the element nodal displacements and slopes.

Thus, for the12 DoFs plate element, one can write:

$\left[w_{12}\right]=\left[P_{12 \times 12}\right][a]$ Equation 33

where $\left[w_{12}\right]$ is the column containing values of displacements and slopes $w, \frac{\partial w}{\partial s}$ and $\frac{\partial w}{\partial t}$ at all four element nodes, establishing the $12 \mathrm{DoFs}$ of the element. The matrix $\left[P_{12 \times 12}\right]$ is obtained by evaluating the polynomial terms in Equation 32 and their appropriate derivatives, at corresponding nodal coordinates. For the square element of length 2 shown in Figure 3 this evaluation yields: 


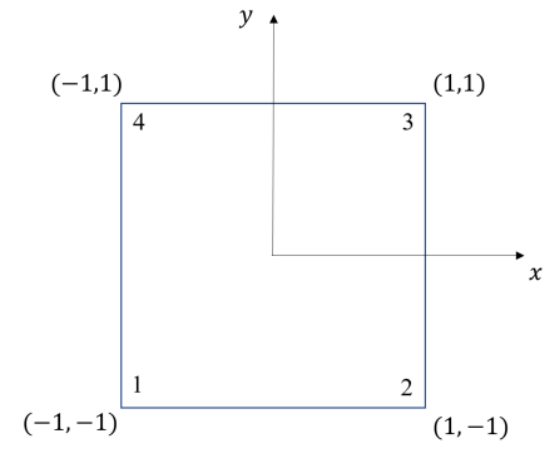

Figure 3 Plate element used for development of finite element

$$
\begin{aligned}
& {\left[P_{12 \times 12}\right]=} \\
& {\left[X_{1}(-1,-1) \quad X_{2}(-1,-1) \quad X_{3}(-1,-1)\right.} \\
& \frac{\partial X_{1}(-1,-1)}{\partial x} \quad \frac{\partial X_{2}(-1,-1)}{\partial x} \quad \frac{\partial X_{3}(-1,-1)}{\partial x} \quad \ldots \\
& \frac{\partial X_{1}(-1,-1)}{\partial y} \quad \frac{\partial X_{2}(-1,-1)}{\partial y} \quad \frac{\partial X_{3}(-1,-1)}{\partial y} \\
& \begin{array}{ccc}
\partial & \ddots
\end{array} \\
& X_{1}(-1,1) \quad X_{2}(-1,1) \quad X_{3}(-1,1) \\
& \frac{\partial X_{1}(-1,1)}{\partial x} \quad \frac{\partial X_{2}(-1,1)}{\partial x} \quad \frac{\partial X_{3}(-1,1)}{\partial x} \\
& \frac{\partial X_{1}(-1,1)}{\partial y} \quad \frac{\partial X_{2}(-1,1)}{\partial y} \quad \frac{\partial X_{3}(-1,1)}{\partial y} \\
& X_{10}(-1,-1) \quad X_{11}(-1,-1) \quad X_{12}(-1,-1) \\
& \frac{\partial X_{10}(-1,-1)}{\partial x} \quad \frac{\partial X_{11}(-1,-1)}{\partial x} \quad \frac{\partial X_{12}(-1,-1)}{\partial x} \\
& \frac{\partial X_{10}(-1,-1)}{\partial y} \quad \frac{\partial X_{11}(-1,-1)}{\partial y} \quad \frac{\partial X_{12}(-1,-1)}{\partial y} \\
& X_{10}(-1,1) \quad X_{11}(-1,1) \quad X_{12}(-1,1) \\
& \frac{\partial X_{10}(-1,1)}{\partial x} \quad \frac{\partial X_{11}(-1,1)}{\partial x} \quad \frac{\partial X_{12}(-1,1)}{\partial x} \\
& \frac{\partial X_{10}(-1,1)}{\partial y} \quad \frac{\partial X_{11}(-1,1)}{\partial y} \quad \frac{\partial X_{12}(-1,1)}{\partial y}
\end{aligned}
$$

Equation 34

Therefore, the vector [a] can be found as:

$[a]=\left[P_{12 \times 12}\right]^{-1}\left[w_{12}\right]$

Equation 35 
Considering this relation, Equation 32 can be rewritten as:

$w(x, y)=[X]\left[P_{12 x 12}\right]^{-1}\left[w_{12}\right]=[N(x, y)]\left[w_{12}\right]$

Vector $[N(x, y)]$ contains the element shape functions which relate displacement function to nodal slopes and displacements. Use of polynomial basis functions for displacement field generates Hermite type shape functions. These shape functions are compared with their DFE counterparts in APPENDIX A.

Using the Hermitian shape functions for flexural displacement, $w$, to derive load-displacement relationship over the element, generates stiffness matrix for static structural problems. This symmetric matrix distributes the stiffness property of the element over its nodal degrees of freedom.

For vibrational analysis, the mass of the element must also be distributed over its DoFs. In consistent mass matrix approach, the distribution of mass is assumed to takes the same form as that of the static stiffness, generating the consistent mass matrix. The mass matrix is a measure of inertia distribution over element DoFs. Vibrational analysis is performed by solving a linear Eigenvalue problem obtained from mass and stiffness matrices.

Development of finite element stiffness matrices can be done in two ways: either by deriving the relationship between assumed displacement function and force vectors using constitutive law, or by application of variational formulations. Both approach yield the same final stiffness matrix.

Galerkin's method is used for finite element development in this chapter, because the methodology stablished here, will serve as a baseline for dynamic finite plate element developed 
in Chapter 5. Development of stiffness plate stiffness matrix using constitutive law can be found in finite element references such as [52].

$\oiint\left(D\left(\frac{\partial^{4} w}{\partial x^{4}}+2 \frac{\partial^{4} w}{\partial x^{2} \partial y^{2}}+\frac{\partial^{4} w}{\partial y^{4}}\right)-\omega^{2} \rho h w\right) \delta w d A=0$

Equation 37

Here, $\delta w$ is weighting function in residual minimization scheme. Since Galerkin's method considers weighting function as identical to the solution function, $\delta w$ can also be interpreted as the virtual displacement consistent with element boundary conditions.

Equation 37 cannot symmetrically describe stiffness for FEM plates, and cannot be used directly. Therefore, further modification of this equation is required.

To do so, two-dimensional Green's theorem for area integrals is applied. This theorem acts in a similar fashion to integration by parts for line integrals [53] by swapping the order of derivation between integrands. Green's theorem is reviewed here for the sake of convenience as it will be used extensively in both FEM and DFE formulations:

For any function $T(x, y)=A(x, y) i+B(x, y) j$ integrated normal to and over a closed boundary the following relations hold true [54]:

$$
\begin{aligned}
& \oint(T . n) d s=\oiint(\nabla . T) d A \\
& \oint((A i+B j) \cdot n) d s=\oint\left(A n_{y}-B n_{x}\right) d s=\oint A d y-B d x \\
& =\oiint(\nabla \cdot(A i+B j)) d A=\oiint\left(\frac{\partial A}{\partial x}+\frac{\partial B}{\partial y}\right) d A
\end{aligned}
$$

Equation 39 
where $n$ is the unit normal vector to the boundary and $d s$ is the differential length along the boundary. The line integrals are performed in counter clockwise positive sense in this theorem.

Introducing Equation 26 as an alternative form of plate governing differential equation, in the weak solution integral of Equation 37, will allow the use of compatibility conditions in manipulating the Galerkin's residual integral by Green's theorem.

$$
\begin{aligned}
& \oiint\left(\frac{\partial^{2} M_{x}}{\partial x^{2}}+2 \frac{\partial^{2} M_{x y}}{\partial x \partial y}+\frac{\partial^{2} M_{y}}{\partial y^{2}}+\omega^{2} \rho h w\right) \delta w d A \\
& =\oiint\left(\left(\frac{\partial^{2} M_{x}}{\partial x^{2}}+\frac{\partial^{2} M_{x y}}{\partial x \partial y}\right)+\left(\frac{\partial^{2} M_{y}}{\partial y^{2}}+\frac{\partial^{2} M_{x y}}{\partial x \partial y}\right)+\omega^{2} \rho h w\right) \delta w d A=0
\end{aligned}
$$

Using Green's theorem on right hand terms of Equation 40 gives:

$$
\begin{aligned}
& \oint\left(\frac{\partial M_{x}}{\partial x}+\frac{\partial M_{x y}}{\partial y}\right) \delta w n_{y} d s \\
& \oiint\left(\frac{\partial^{2} M_{x}}{\partial x^{2}}+\frac{\partial^{2} M_{x y}}{\partial x \partial y}\right) \delta w d A+\oiint\left(\frac{\partial M_{x}}{\partial x}+\frac{\partial M_{x y}}{\partial y}\right) \frac{\partial \delta w}{\partial x} d A \\
- & \oint\left(\frac{\partial M_{y}}{\partial y}+\frac{\partial M_{x y}}{\partial x}\right) \delta w n_{x} d s \\
= & \oiint\left(\frac{\partial^{2} M_{y}}{\partial y^{2}}+\frac{\partial^{2} M_{x y}}{\partial x \partial y}\right) \delta w d A+\oiint\left(\frac{\partial M_{y}}{\partial y}+\frac{\partial M_{x y}}{\partial x}\right) \frac{\partial \delta w}{\partial y} d A
\end{aligned}
$$

Substituting similar terms from Equation 41 and Equation 42 in Equation 40 results in: 
$\oiint\left(\frac{\partial^{2} M_{x}}{\partial x^{2}}+2 \frac{\partial^{2} M_{x y}}{\partial x \partial y}+\frac{\partial^{2} M_{y}}{\partial y^{2}}+\omega^{2} \rho h w\right) \delta w d A$

$=\oint\left(\frac{\partial M_{x}}{\partial x}+\frac{\partial M_{x y}}{\partial y}\right) \delta w n_{y} d s-\oiint\left(\frac{\partial M_{x}}{\partial x}+\frac{\partial M_{x y}}{\partial y}\right) \frac{\partial \delta w}{\partial x} d A$

$-\oint\left(\frac{\partial M_{y}}{\partial y}+\frac{\partial M_{x y}}{\partial x}\right) \delta w n_{x} d s-\oiint\left(\frac{\partial M_{y}}{\partial y}+\frac{\partial M_{x y}}{\partial x}\right) \frac{\partial \delta w}{\partial y} d A$

$+\oiint\left(\omega^{2} \rho h w\right) \delta w d A$

Equation 43

Comparing Equation 43 with shear flow definitions developed previously for thin plates (Equation 24 and Equation 25) gives:

$$
\begin{aligned}
& \oiint\left(\frac{\partial^{2} M_{x}}{\partial x^{2}}+2 \frac{\partial^{2} M_{x y}}{\partial x \partial y}+\frac{\partial^{2} M_{y}}{\partial y^{2}}+\omega^{2} \rho h w\right) \delta w d A \\
& =\oint Q_{x} \delta w n_{y} d s-\oint Q_{y} \delta w n_{x} d s-\oiint\left(\frac{\partial M_{x}}{\partial x}+\frac{\partial M_{x y}}{\partial y}\right) \frac{\partial \delta w}{\partial x} d A \\
& -\oiint\left(\frac{\partial M_{y}}{\partial y}+\frac{\partial M_{x y}}{\partial x}\right) \frac{\partial \delta w}{\partial y} d A+\oiint\left(\omega^{2} \rho h w\right) \delta w d A
\end{aligned}
$$

Equation 44

The terms $\int Q_{x} \delta w n_{y} d s$ and $\int Q_{y} \delta w n_{x} d s$, represent virtual work done by shear forces corresponding to shear flows $Q_{x}$ and $Q_{y}$, through virtual displacement $\delta w$, along the exterior edges of the plate boundaries. For a free vibrating plate, the boundaries are either clamped, pinned or fixed. In all of these conditions, a plate has either no shear force or, in order to be compliant with boundary conditions, the virtual displacement $\delta w$ must be zero. Hence these terms will vanish from the equation.

$-\oiint\left(\frac{\partial M_{x}}{\partial x}\right) \frac{\partial \delta w}{\partial x} d A-\oiint\left(\frac{\partial M_{x y}}{\partial y}\right) \frac{\partial \delta w}{\partial x} d A-\oiint\left(\frac{\partial M_{y}}{\partial y}\right) \frac{\partial \delta w}{\partial y} d A$

$-\oiint\left(\frac{\partial M_{x y}}{\partial x}\right) \frac{\partial \delta w}{\partial y} d A+\oiint\left(\omega^{2} \rho h w\right) \delta w d A=0$

Equation 45 
Now again invoking Green's theorem, results:

$$
\begin{gathered}
-\oint\left(M_{x}\right) \frac{\partial \delta w}{\partial x} n_{y} d s=-\oiint\left(\frac{\partial M_{x}}{\partial x}\right) \frac{\partial \delta w}{\partial x} d A-\oiint\left(M_{x}\right) \frac{\partial^{2} \delta w}{\partial x^{2}} d A \\
\oint\left(M_{y}\right) \frac{\partial \delta w}{\partial x} n_{x} d s=-\oiint\left(\frac{\partial M_{y}}{\partial y}\right) \frac{\partial \delta w}{\partial y} d A-\oiint\left(M_{y}\right) \frac{\partial^{2} \delta w}{\partial y^{2}} d A \\
\oint\left(M_{x y}\right) \frac{\partial \delta w}{\partial x} n_{x} d s=-\oiint\left(\frac{\partial M_{x y}}{\partial y}\right) \frac{\partial \delta w}{\partial x} d A-\oiint\left(M_{x y}\right) \frac{\partial^{2} \delta w}{\partial x \partial y} d A \\
-\oint\left(M_{x y}\right) \frac{\partial \delta w}{\partial y} n_{y} d s=-\oiint\left(\frac{\partial M_{x y}}{\partial x}\right) \frac{\partial \delta w}{\partial y} d A \\
-\oiint\left(M_{x y}\right) \frac{\partial^{2} \delta w}{\partial x \partial y} d A
\end{gathered}
$$

Applying Equation 46 through 49 to Equation 45 results in:

$$
\begin{aligned}
& \oiint\left(M_{x}\right) \frac{\partial^{2} \delta w}{\partial x^{2}} d A-\oint\left(M_{x}\right) \frac{\partial \delta w}{\partial x} n_{y} d s+\oint\left(M_{x y}\right) \frac{\partial \delta w}{\partial x} n_{x} d s \\
& +\oiint\left(M_{x y}\right) \frac{\partial^{2} \delta w}{\partial x \partial y} d A+\oint\left(M_{y}\right) \frac{\partial \delta w}{\partial x} n_{x} d s+\oiint\left(M_{y}\right) \frac{\partial^{2} \delta w}{\partial y^{2}} d A \\
& -\oint\left(M_{x y}\right) \frac{\partial \delta w}{\partial y} n_{y} d s+\oiint\left(M_{x y}\right) \frac{\partial^{2} \delta w}{\partial x \partial y} d A+\oiint\left(\omega^{2} \rho h w\right) \delta w d A=0
\end{aligned}
$$

Here the terms $\oint\left(\mathrm{M}_{\mathrm{x}}\right) \frac{\partial \delta w}{\partial x} n_{y} d s, \oint\left(\mathrm{M}_{\mathrm{xy}}\right) \frac{\partial \delta w}{\partial x} n_{x} d s, \oint\left(\mathrm{M}_{\mathrm{y}}\right) \frac{\partial \delta w}{\partial x} n_{x} d s$ and $\oint\left(\mathrm{M}_{\mathrm{xy}}\right) \frac{\partial \delta w}{\partial y} n_{y} d s$ show the virtual work done by moments corresponding to $M_{x}, M_{y}$ and $M_{x y}$ through virtual slopes $\frac{\partial \delta w}{\partial x}$ and $\frac{\partial \delta w}{\partial y}$ at the plate boundaries. Once again, for a freely vibrating thin plate, at any boundary condition, either the moments or the virtual slopes are zero, and hence their product yield zero virtual work: 


$$
\begin{aligned}
& \oiint\left(\left(M_{x}\right) \frac{\partial^{2} \delta w}{\partial x^{2}}+2\left(M_{x y}\right) \frac{\partial^{2} \delta w}{\partial x \partial y}+\left(M_{y}\right) \frac{\partial^{2} \delta w}{\partial y^{2}}\right) d A \\
& +\oiint\left(\omega^{2} \rho h w\right) \delta w d A=0
\end{aligned}
$$

Equation 51

Substituting definitions of $M_{x}, M_{y}$ and $M_{x y}$ from chapter 2 yields:

$$
\begin{aligned}
& D \oiint\left(\frac{\partial^{2} w}{\partial x^{2}}\right) \frac{\partial^{2} \delta w}{\partial x^{2}}+\left(v \frac{\partial^{2} w}{\partial y^{2}}\right) \frac{\partial^{2} \delta w}{\partial x^{2}}+\left(\frac{\partial^{2} w}{\partial y^{2}}\right) \frac{\partial^{2} \delta w}{\partial y^{2}} \\
& \left.+\left(v \frac{\partial^{2} w}{\partial x^{2}}\right) \frac{\partial^{2} \delta w}{\partial y^{2}}+2\left((1-v) \frac{\partial^{2} w}{\partial x \partial y}\right) \frac{\partial^{2} \delta w}{\partial x \partial y}\right) d A \\
& -\oiint\left(\omega^{2} \rho h w\right) \delta w d A=0
\end{aligned}
$$

By using Hermitian distribution of Equation 36 for the displacement function $w$ and the virtual displacement $\delta w$, the finite element formulation for 12 DoF square plate of Figure 3 is obtained.

$$
\begin{aligned}
& \int_{-1}^{1} \int_{-1}^{1} D\left(\left(\frac{\partial^{2}\left[N^{T}\right]}{\partial x^{2}}\right) \frac{\partial^{2}[N]}{\partial x^{2}}+\left(v \frac{\partial^{2}\left[N^{T}\right]}{\partial y^{2}}\right) \frac{\partial^{2}[N]}{\partial x^{2}}+\left(\frac{\partial^{2}\left[N^{T}\right]}{\partial y^{2}}\right) \frac{\partial^{2}[N]}{\partial y^{2}}\right. \\
& \left.+\left(v \frac{\partial^{2}\left[N^{T}\right]}{\partial x^{2}}\right) \frac{\partial^{2}[N]}{\partial y^{2}}+2\left((1-v) \frac{\partial^{2}\left[N^{T}\right]}{\partial x \partial y}\right) \frac{\partial^{2}[N]}{\partial x \partial y}\right) d x d y \\
& -\omega^{2} \int_{-1}^{1} \int_{-1}^{1}\left(\rho h\left[N^{T}\right]\right)[N] d x d y=0
\end{aligned}
$$

Equation 53

The first area integral results in the formation of the stiffness matrix $[K]$ and the second area integral generates mass matrix $[\mathrm{m}]$. The terms $N$ and $N^{T}$ shows the shape function matrix of 12DoF FEM plate and its transpose. Notice that the stiffness matrix thus obtained is by definition symmetric. By confining the stiffness and mass matrix on DoFs restricted by boundary conditions, different vibrational problems can be solved with application of Eigenvalue analysis. 


\section{THIN PLATE DYNAMIC SOLUTION}

The DSM plate developed by Casimira et al. [45], involved dynamic solution of the plate problem. Since the researchers incorporated complete solution in their formulation, the plate element required solving series mathematics. Although their method generated accurate solution, the results were case specific.

While similar development of the DFE matrices, incorporating infinite series solutions, can generate very accurate results, it restricts the applicability of the formulation to specific geometries and will be computationally expensive. Instead, in this study, subsets of the solution space have been investigated to produce a dynamic finite plate element comparable to finite element plates.

The aim here is to study the performance of dynamic finite elements having finite size, developed from plate vibration solution subspace. As the complete solution space is not incorporated in element formulation, the DFE is expected not to have exact solution, and to rather converge to analytical results.

The finite element formulation developed in the last chapter incorporates polynomial basis functions assumption in the modeling the lateral displacement field $w$. In this chapter, instead of the classic FEM polynomials, dynamic solutions are proposed for modelling flexural displacement $w$. These solutions will satisfy the Kirchhoff plate model for harmonic oscillation case (Equation 31) including the dynamic force term. Therefore, these functions in addition to dependency on plate coordinates, will also depend on vibration frequency $\omega$. 
Using the property of infinite dimensionality of the Hilbert space generated by solution of the partial differential equations, one can generate multiple solutions that satisfy the governing differential equation of the plate. However, to develop a $12 \mathrm{DoF}$ vibrational plate element for comparison with the FEM plate developed in last chapter, 12 linearly independent vectors of the solution space are required.

Although by using Fourier series solutions available for partial differential equations such solutions can be obtained, in this study, separation of variables along with ordinary differential equation solution methods were applied instead. The reason for this approach was to obtain general solution sets based on specific physical requirements, such as appropriate distribution of dynamic force over element curvatures and dynamic definitions of internal moments and loads that could not be achieved by FEM formulation.

Besides physical restrictions, only solution sets that satisfy following two conditions are considered for plate DFE developed in this research:

1. Are symmetric with respect to $x$ and $y$

2. $\quad$ Are $C^{\infty}$ continuous

To obtain such solutions, invoking separation of variable assumes:

$w=\mathcal{G}(x) H(y)$ Equation 54

Substituting Equation 54 in Equation 31 yields:

$\left(H(y) \frac{\partial^{4} \mathcal{G}(x)}{\partial x^{4}}+2 \frac{\partial^{2} \mathcal{G}(x) \partial^{2} H(y)}{\partial x^{2} \partial y^{2}}+\mathcal{G}(x) \frac{\partial^{4} H(y)}{\partial y^{4}}\right)=\frac{\rho h \omega^{2}}{D} \mathcal{G}(x) H(y)$

Equation 55 
In following subsections, three possible solution sets are presented, and their physical interpretation and applicability to the development of dynamic finite element formulation as basis functions is discussed.

\subsection{SOLUTION SET 1}

A possible solution set can be obtained by distributing the term $\frac{\rho h \omega^{2}}{D}$ evenly between each term on the left side of Equation 55 which leads to formation ordinary differential equations. Please note that these terms represent the partial derivatives $\frac{\partial^{2} M_{x}}{\partial x^{2}}, \frac{\partial^{2} M_{x y}}{\partial x \partial y}$ and $\frac{\partial^{2} M_{y}}{\partial y^{2}}$ (see Equation 26 through 30). Since the term $\frac{\rho h \omega^{2}}{D}$ can be interpreted as dynamic force using D'Alembert principle, this distribution assumes equal share of the dynamic load over bending movement $M_{x}$, $M_{y}$ and twisting moment $M_{x y}$ derivatives.

$H(y) \frac{\partial^{4} \mathcal{G}(x)}{\partial x^{4}}=\frac{\partial^{2} \mathcal{G}(x) \partial^{2} H(y)}{\partial x^{2} \partial y^{2}}=\mathcal{G}(x) \frac{\partial^{4} H(y)}{\partial y^{4}}=\frac{\frac{\rho h \omega^{2}}{D} \mathcal{G}(x) H(y)}{4}$

Equation 56

The equalities $H(y) \frac{\partial^{4} \mathcal{G}(x)}{\partial x^{4}}=\frac{\frac{\rho h \omega^{2}}{D} \mathcal{G}(x) H(y)}{4}$ and $\mathcal{G}(x) \frac{\partial^{4} H(y)}{\partial y^{4}}=\frac{\frac{\rho h \omega^{2}}{D} \mathcal{G}(x) H(y)}{4}$, from the above equation, can be solved as ordinary differential equations in $x$ and $y$ resulting in Equation 57 and Equation 58.

$\frac{\partial^{4} \mathcal{G}(x)}{\partial x^{4}}=\frac{\frac{\rho h \omega^{2}}{D} \mathcal{G}(x)}{4}$

Equation 57 
$\frac{\partial^{4} H(y)}{\partial y^{4}}=\frac{\frac{\rho h \omega^{2}}{D} H(y)}{4}$

Equation 58

The solutions to these equations are of the form:

$H(y)=e^{ \pm\left(\frac{\rho h \omega^{2}}{D}\right)^{\frac{1}{4}} y}$, and $e^{ \pm i\left(\frac{\rho h \omega^{2}}{D}\right)^{\frac{1}{4}} y}$

Equation 59

$\mathcal{G}(x)=e^{ \pm\left(\frac{\frac{\rho h \omega^{2}}{D}}{4}\right)^{\frac{1}{4}} x}$, and $e^{ \pm i\left(\frac{\rho h \omega^{2}}{D}\right)^{\frac{1}{4}} x}$

Equation 60

Equation 59 and Equation 60 can be combined to produce 16 different solution functions for $w$. However, the equality $\frac{\partial^{2} \mathcal{G}(x) \partial^{2} H(y)}{\partial x^{2} \partial y^{2}}=\frac{\frac{\rho h \omega^{2}}{D} G(x) H(y)}{4}$, in Equation 56 restricts which combinations can be used as solutions for the plate equation. This term is satisfied when solutions with only real or imaginary exponent of $\mathcal{G}(x)$ and $H(y)$ are combined. Combinations such as $w(x, y)=$ $\left(e^{ \pm\left(\frac{\rho h \omega^{2}}{{ }^{2}}\right)^{\frac{1}{4}} x}\right)\left(e^{ \pm i\left(\frac{\rho h \omega^{2}}{{ }^{\frac{1}{4}}}\right)^{\frac{1}{4}} y}\right)$ where real and imaginary exponents of $\mathcal{G}(x)$ and $H(y)$ are used, do not satisfy this term and therefore do not satisfy the plate equation. As a result, this method generates only 8 solutions which are not sufficient for modelling of a plate element. 


\subsection{SOLUTION SET 2}

Another set of solutions to Equation 56 can be found when the partial differential equation is directly reduced to one variable ordinary differential equation. For instance, consider the following reduction:

$\frac{\partial^{4} H(y)}{\partial y^{4}}=\frac{\rho h \omega^{2}}{D} H(y)$

Equation 61

The generic solution to such equation is of the form $H(y)=e^{m y}$ and can be found with ease. The viable $\mathcal{G}(x)$ functions can be determined through the restrictions that made the reduction of Equation 56 to Equation 61 possible in the first place:

$H(y) \frac{\partial^{4} \mathcal{G}(x)}{\partial x^{4}}+2 \frac{\partial^{2} \mathcal{G}(x) \partial^{2} H(y)}{\partial x^{2} \partial y^{2}}=0$

Equation 62

Equation 62 can be satisfied directly if each of the terms involved becomes zero for all values of $x$. Hence:

$\frac{\partial^{4} \mathcal{G}(x)}{\partial x^{4}}=\frac{\partial^{2} \mathcal{G}(x)}{\partial x^{2}}=0$

Equation 63

Equation 63 is an ordinary differential equation in variable $x$ and is satisfied by linear functions of $x$. Therefore, the displacement function can be written as:

$w(x, y)=(a+b x)\left(e^{ \pm\left(\frac{\rho h \omega^{2}}{D}\right)^{\frac{1}{4}} y}\right)$, and $(a+b x)\left(e^{ \pm i\left(\frac{\rho h \omega^{2}}{D}\right)^{\frac{1}{4}} y}\right)$ 
In the development of Equation 64, the dynamic force was devoted to only $y$ direction derivative and therefore to radius of curvature $r_{y}$. The linear function $\mathcal{G}(x)$ is nullified in differentiation of order two and higher. Therefore, the curvature $r_{x}$ (Equation 2) receives no share of the dynamic load for these solutions and cannot be captured. Hence this solution set reduces the plate behaviour to a one dimension beam like model.

The same approach can be applied to generate a counterpart for Equation 64 that is mirrored around the line $y=x$.

$w(x, y)=(c+d y)\left(e^{ \pm\left(\frac{\rho h \omega^{2}}{D}\right)^{\frac{1}{4}} x}\right)$, and $(c+d y)\left(e^{ \pm i\left(\frac{\rho h \omega^{2}}{D}\right)^{\frac{1}{4}} x}\right)$

Equation 65

Similar to Equation 64, here the curvature $r_{y}$ cannot be captured. The pair of these two solution sets is both $C^{\infty}$ continuous and symmetric with respect to $x$ and $y$.

Equation 64 and Equation 65 generate 16 sets of independent solutions for the governing differential equation (Equation 30) :

$$
\begin{aligned}
& \left(e^{\left(\frac{\rho h \omega^{2}}{D}\right)^{ \pm \frac{1}{4}} y}\right),\left(e^{\left(\frac{\rho h \omega^{2}}{D}\right)^{ \pm i \frac{1}{4}} y}\right),(x)\left(e^{\left(\frac{\rho h \omega^{2}}{D}\right)^{ \pm \frac{1}{4}} y}\right),(x)\left(e^{\left(\frac{\rho h \omega^{2}}{D}\right)^{ \pm i \frac{1}{4}} y}\right), \\
& \left(e^{\left(\frac{\rho h \omega^{2}}{D}\right)^{\frac{1}{ \pm 4}} x}\right),\left(e^{\left(\frac{\rho h \omega^{2}}{D}\right)^{ \pm i \frac{1}{4}} x}\right),(y)\left(e^{\left(\frac{\rho h \omega^{2}}{D}\right)^{ \pm \frac{1}{4}} x}\right),(y)\left(e^{\left(\frac{\rho h \omega^{2}}{D}\right)^{ \pm i \frac{1}{4}} x}\right)
\end{aligned}
$$

Equation 66

The solutions obtained here is similar to basis functions used for development of the beam dynamic finite element formulation and are not able to fully model plate specific behaviour 
related to coupling of $x$ and $y$ directions. These governing differential equationsolutions can be written as:

$\cos \left(\gamma_{x y} x\right), \cos \left(\gamma_{x y} y\right), \sin \left(\gamma_{x y} x\right), \sin \left(\gamma_{x y} y\right), \cosh \left(\gamma_{x y} x\right), \cosh \left(\gamma_{x y} y\right)$, $\sinh \left(\gamma_{x y} x\right), \sinh \left(\gamma_{x y} y\right), y \cos \left(\gamma_{x y} x\right), x \cos \left(\gamma_{x y} y\right), y \sin \left(\gamma_{x y} x\right)$, $x \sin \left(\gamma_{x y} y\right), y \cosh \left(\gamma_{x y} x\right), x \cosh \left(\gamma_{x y} y\right), y \sinh \left(\gamma_{x y} x\right), x \sinh \left(\gamma_{x y} y\right)$

where $\gamma_{x y}=\left(\frac{\rho h \omega^{2}}{D}\right)^{\frac{1}{4}}$

\subsection{SOLUTION SET 3}

Another way of satisfying Equation 62 is by finding solutions that make the summation of the terms equal to zero. Assuming $\mathcal{G}(x)=e^{n x}$ gives:

$n^{4}+2 n^{2} m^{2}=0$

Equation 68

Therefore, for non-trivial solution, one gets $n^{2}=-2 m^{2}$. By performing similar approach mirrored about $x$ and $y, 16$ basis functions obtained from this solution set are:

$\left(e^{\sqrt{2}\left(\frac{\rho h \omega^{2}}{D}\right)^{ \pm i \frac{1}{4}} x}\right)\left(e^{\left(\frac{\rho h \omega^{2}}{D}\right)^{ \pm \frac{1}{4}} y}\right),\left(e^{\sqrt{2}\left(\frac{\rho h \omega^{2}}{D}\right)^{ \pm \frac{1}{4}} x}\right)\left(e^{\left(\frac{\rho h \omega^{2}}{D}\right)^{ \pm i \frac{1}{4}} y}\right)$
$\left(e^{\left(\frac{\rho h \omega^{2}}{D}\right)^{ \pm \frac{1}{4}} x}\right)\left(e^{\sqrt{2}\left(\frac{\rho h \omega^{2}}{D}\right)^{ \pm i \frac{1}{4}} y}\right),\left(e^{\sqrt{2}\left(\frac{\rho h \omega^{2}}{D}\right)^{ \pm \frac{1}{4}} y}\right)\left(e^{\left(\frac{\rho h \omega^{2}}{D}\right)^{ \pm i \frac{1}{4}} x}\right)$

Equation 69

Because similar to the solution set 2, these solutions also satisfy Equation 61, the dynamic force is still fully distributed over $\frac{\partial^{2} M_{y}}{\partial y^{2}}$ derivative. Equation 68 implies that for these solutions $\frac{\partial^{2} M_{x}}{\partial x^{2}}=$ 
$-2 \frac{\partial^{2} M_{y}}{\partial y^{2}}$, which in turn assigns a pseudo counter dynamic force to $\frac{\partial^{2} M_{x}}{\partial x^{2}}$ that is twice the actual vibration induced dynamic force and makes this loading very large. This loading is counteracted by twisting loading $\frac{\partial^{2} M_{x y}}{\partial x \partial y}$ which also receives large pseudo contribution of the dynamic force. 


\section{DYNAMIC FINITE PLATE ELEMENT DEVELOPMENT}

By using the basis functions developed in the previous chapter, the development of the dynamic finite element matrices can be carried out. Because these functions depend on vibrational frequency, the resulting element matrices are also frequency dependent. This is unlike the classic finite element formulation were both stiffness and mass matrices are constants.

Hence, similar to FEM, first step is to find the element shape functions in order to relate the lateral displacement $w$ to nodal DoF values. For the plate element in Figure 4, this can be done by evaluating matrix $[P]$ (see chapter 3, Equation 34) over element nodal coordinates using the dynamic basis functions:

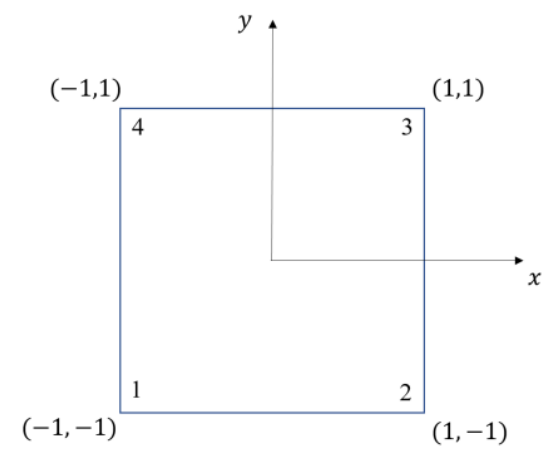

Figure 4 Plate element used for development of DFE 


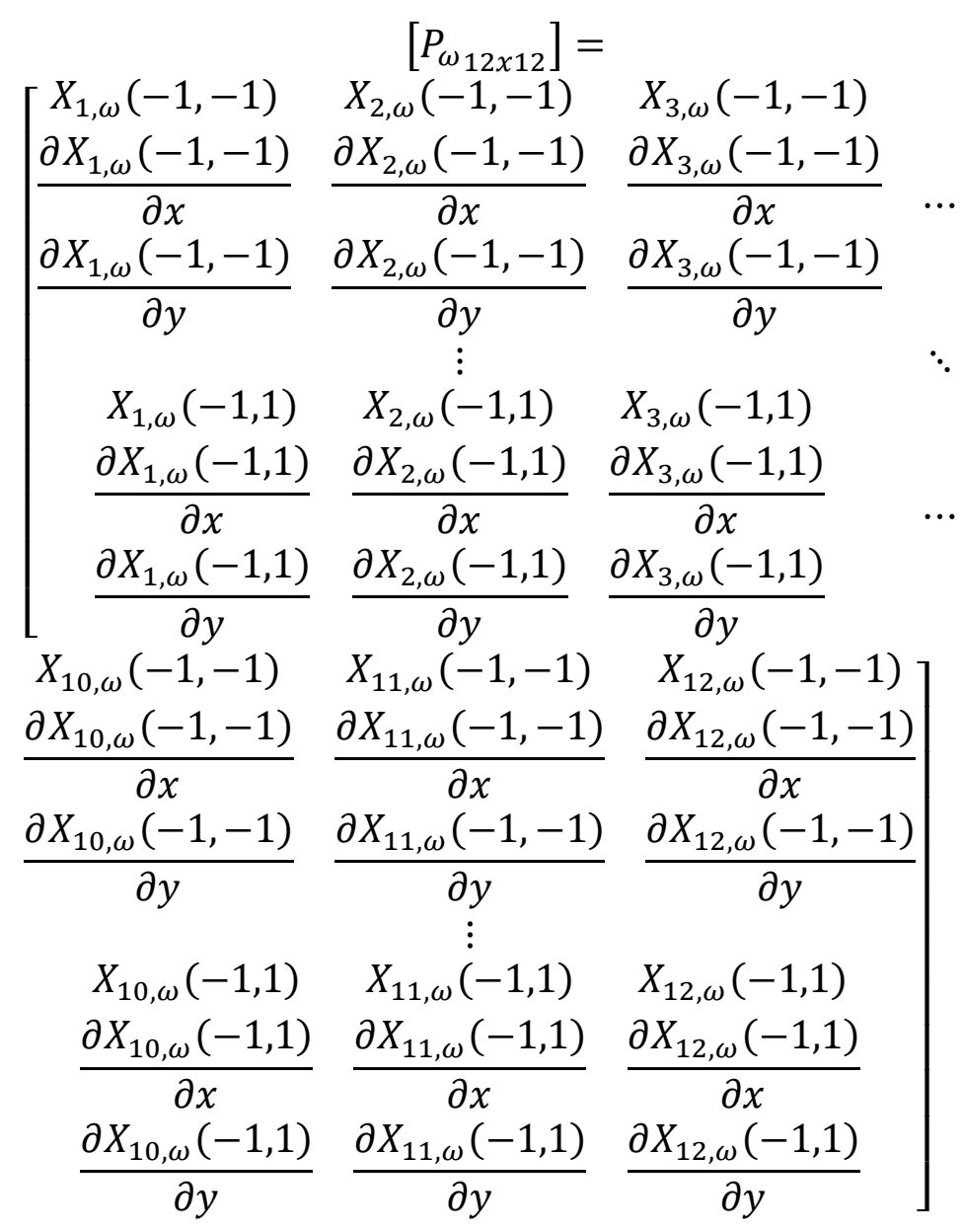

Equation 70

The simplest plate vibrational elements are three node triangles with three DoFs per node, which require nine linearly independent basis functions for the lateral displacement field, $w$ [55]. Therefore, because for development of any plate element at least nine linearly independent basis functions are required, from the solution sets presented in chapter 4 , solution set 1 cannot be used for plate DFE development.

While solution set 3 does generate enough basis functions, the DFE developed from this set generated poor results. This solution set has thus been discarded from further investigation and the element development is not reported here. 
Solution set 2 generates 16 different basis functions as well. Although these functions are linearly independent in a continuous space, when numerically evaluated in Equation 70 to obtain $\left[P_{\omega}\right]$ for an element domain such as that of Figure 4, the numerical columns were found to show linear dependency.

To better demonstrate the linear dependency problem, consider the four dynamic basis functions, $\cos \left(\gamma_{x y} x\right), \cos \left(\gamma_{x y} y\right), \cosh \left(\gamma_{x y} x\right)$ and $\cosh \left(\gamma_{x y} y\right)$ to represent $X_{1, \omega}, X_{2, \omega}, X_{3, \omega}$, and $X_{4, \omega}$ respectively, generating the left four columns of matrix $\left[P_{\omega}\right]$. It can be shown that when matrix $\left[P_{\omega}\right]$ is calculated over nodal points of the plate element of Figure 4, for these basis functions the following relation holds true:

$$
\left[c_{4}\right]=\left[c_{3}\right]+\left(\frac{\sinh \left(\gamma_{x y}\right)}{\sin \left(\gamma_{x y}\right)}\right)\left[c_{1}\right]-\left(\frac{\sinh \left(\gamma_{x y}\right)}{\sin \left(\gamma_{x y}\right)}\right)\left[c_{2}\right]
$$

Equation 71

where $\left[c_{i}\right]$ is the $i$ th column vector of matrix $\left[P_{\omega}\right]$. Linear independency of the column vectors of matrix $\left[P_{\omega}\right]$ is necessary to allow shape function definition. Without such independency, this matrix will not be invertible and a unique relation between flexural displacement $w$ and nodal degrees of freedom cannot be achieved.

To eliminate this dependency, the 16 basis functions of solution set 3 are combined to generate a set of 12 new mixed basis functions. These basis functions are generated such that in the limiting case, where vibrational frequency approaches zero, the resulting shape functions will approach the 12 DoF polynomial shape functions. 


$$
\begin{aligned}
& X_{\omega} 1=\cos \left(\gamma_{x y} x\right)+\cos \left(\gamma_{x y} y\right) \\
& X_{\omega} 2=x \cos \left(\gamma_{x y} y\right) \\
& X_{\omega} 3=y \cos \left(\gamma_{x y} x\right) \\
& X_{\omega} 4=\frac{\cosh \left(\gamma_{x y} x\right)-\cos \left(\gamma_{x y} x\right)}{\gamma_{x y}^{2}} \\
& X_{\omega} 5=\frac{x \sin \left(\gamma_{x y} y\right)+y \sin \left(\gamma_{x y} x\right)}{\gamma_{x y}} \\
& X_{\omega} 6=\frac{\cosh \left(\gamma_{x y} y\right)-\cos \left(\gamma_{x y} y\right)}{\gamma_{x y}^{2}} \\
& X_{\omega} 7=\frac{\sinh \left(\gamma_{x y} x\right)-\sin \left(\gamma_{x y} x\right)}{\gamma_{x y}^{3}} \\
& X_{\omega} 8=\frac{(y) \cosh \left(\gamma_{x y} x\right)-(y) \cos \left(\gamma_{x y} x\right)}{\gamma_{x y}^{2}} \\
& X_{\omega} 9=\frac{(x) \cosh \left(\gamma_{x y} y\right)-(x) \cos \left(\gamma_{x y} y\right)}{\gamma_{x y}^{2}} \\
& X_{\omega} 10=\frac{\sinh \left(\gamma_{x y} y\right)-\sin \left(\gamma_{x y} y\right)}{\gamma_{x y}^{3}} \\
& X_{\omega} 11=\frac{(y) \sinh \left(\gamma_{x y x}\right)-(y) \sin \left(\gamma_{x y} x\right)}{\gamma_{x y}^{3}} \\
& X_{\omega} 12=\frac{(x) \sinh \left(\gamma_{x y} y\right)-(x) \sin \left(\gamma_{x y} y\right)}{\gamma_{x y}^{3}}
\end{aligned}
$$

Here, the basis function $X_{i}$ depends on the vibrational frequency $\omega$ through the relation $\gamma_{x y}=$ $\left(\frac{\rho h \omega^{2}}{D}\right)^{\frac{1}{4}}$ and the vector

$\left[X_{\omega}\right]=\left[X_{\omega} 1, X_{\omega} 2, X_{\omega} 3, X_{\omega} 4, X_{\omega} 5, X_{\omega} 6, X_{\omega} 7, X_{\omega} 8, X_{\omega} 9, X_{\omega} 10, X_{\omega} 11, X_{\omega} 12\right] \quad$ reduces $\quad$ to $\left[2, x, y, x^{2}, 2 x y, y^{2}, \frac{x^{3}}{3}, x^{2} y, x y^{2}, \frac{y^{3}}{3}, \frac{x^{3} y}{3}, \frac{x y^{3}}{3}\right]$ when $\lim _{\omega \rightarrow 0}\left(\left[X_{\omega}\right]\right)$ is applied.

Since the basis functions used in this process are solutions of the governing differential equation of the plate, the resulting dynamic finite element formulation will be an intermediate, between the analytical solution and finite element technique. 
Because DFE involves trigonometric and hyperbolic basis functions, performing area integrals for arbitrary shapes would be very difficult. Further difficulty arises when mapping these integral to a reference coordinate system, which requires introduction of absolute value of mapping Jacobian matrix determinant. Numerical integration techniques such as Gauss quadrature formula have been used in such situation for FEM plates with Hermitian polynomial shape functions, but use of such methods for DFE formulations will result in lost accuracy and extra computational overhead.

Use of plate equation solutions in development of DFE, allows further simplification of Galerkin's residual minimization method to line integrals, over the boundaries of the element. Line integrals produced from reduction of Galerkin's methods can be readily transformed to natural coordinate system for plate of any shape using variable substitution and the integrals can be performed analytically without loss in accuracy.

Simplification of Galerkin's residual minimization method in development of plate DFE relies on the use of Green's theorem. This reduction is similar to use of integration by parts in beam DFE development, and is essential for extension of DFE plates to arbitrary geometries.

Green's theorem is applied two times to the terms of Equation 52, with respect to the variable by which $w$ is differentiated. For the first term in Equation 52 the following relation is produced:

$$
\begin{aligned}
& \oiint\left(\frac{\partial^{2} w}{\partial x^{2}}\right) \frac{\partial^{2} \delta w}{\partial x^{2}} d A=\oint\left(\frac{\partial w}{\partial x}\right) \frac{\partial^{2} \delta w}{\partial x^{2}} n_{y} d s-\oiint\left(\frac{\partial w}{\partial x}\right) \frac{\partial^{3} \delta w}{\partial x^{3}} d A \\
& \oiint\left(\frac{\partial^{2} w}{\partial x^{2}}\right) \frac{\partial^{2} \delta w}{\partial x^{2}} d A=\oint\left(\frac{\partial w}{\partial x}\right) \frac{\partial^{2} \delta w}{\partial x^{2}} n_{y} d s-\oint(w) \frac{\partial^{3} \delta w}{\partial x^{3}} n_{y} d s \\
& +\oiint(w) \frac{\partial^{4} \delta w}{\partial x^{4}} d A
\end{aligned}
$$

Equation 74 
Applying the same procedure to the second, third and fourth term generates:

$\oiint\left(\frac{\partial^{2} w}{\partial y^{2}}\right) \frac{\partial^{2} \delta w}{\partial x^{2}} d A=-\oint\left(\frac{\partial w}{\partial y}\right) \frac{\partial^{2} \delta w}{\partial x^{2}} n_{x} d s+\oint(w) \frac{\partial^{3} \delta w}{\partial y \partial x^{2}} n_{x} d s$

$+\oiint(w) \frac{\partial^{4} \delta w}{\partial y^{2} \partial x^{2}} d A$

Equation 75

$\oiint\left(\frac{\partial^{2} w}{\partial y^{2}}\right) \frac{\partial^{2} \delta w}{\partial y^{2}} d A=-\oint\left(\frac{\partial w}{\partial y}\right) \frac{\partial^{2} \delta w}{\partial y^{2}} n_{x} d s+\oint(w) \frac{\partial^{3} \delta w}{\partial y^{3}} n_{x} d s$

$+\oiint(w) \frac{\partial^{4} \delta w}{\partial y^{4}} d A$

Equation 76

$\oiint\left(\frac{\partial^{2} w}{\partial x^{2}}\right) \frac{\partial^{2} \delta w}{\partial y^{2}} d A=\oint\left(\frac{\partial w}{\partial x}\right) \frac{\partial^{2} \delta w}{\partial y^{2}} n_{y} d s-\oint(w) \frac{\partial^{3} \delta w}{\partial x \partial y^{2}} n_{y} d s$

$+\oiint(w) \frac{\partial^{4} \delta w}{\partial x^{2} \partial y^{2}} d A$

Equation 77

Therefore, Equation 52 is rewritten as:

$D\left[\oint\left(\frac{\partial w}{\partial x}\right) \frac{\partial^{2} \delta w}{\partial x^{2}} n_{y} d s-\oint(w) \frac{\partial^{3} \delta w}{\partial x^{3}} n_{y} d s+\oiint(w) \frac{\partial^{4} \delta w}{\partial x^{4}} d A\right.$

$-v \oint\left(\frac{\partial w}{\partial y}\right) \frac{\partial^{2} \delta w}{\partial x^{2}} n_{x} d s+v \oint(w) \frac{\partial^{3} \delta w}{\partial y \partial x^{2}} n_{x} d s$

$+v \oiint(w) \frac{\partial^{4} \delta w}{\partial y^{2} \partial x^{2}} d A-\oint\left(\frac{\partial w}{\partial y}\right) \frac{\partial^{2} \delta w}{\partial y^{2}} n_{x} d s$

$+\oint(w) \frac{\partial^{3} \delta w}{\partial y^{3}} n_{x} d s+\oiint(w) \frac{\partial^{4} \delta w}{\partial y^{4}} d A$

$+v \oint\left(\frac{\partial w}{\partial x}\right) \frac{\partial^{2} \delta w}{\partial y^{2}} n_{y} d s-v \oint(w) \frac{\partial^{3} \delta w}{\partial x \partial y^{2}} n_{y} d s+v \oiint(w) \frac{\partial^{4} \delta w}{\partial x^{2} \partial y^{2}} d A$

$\left.+\oiint\left((1-v) \frac{\partial^{2} w}{\partial x \partial y}\right) \frac{\partial^{2} \delta w}{\partial x \partial y} d A+\oiint\left((1-v) \frac{\partial^{2} w}{\partial x \partial y}\right) \frac{\partial^{2} \delta w}{\partial x \partial y} d A\right]$

$-\oiint\left(\omega^{2} \rho h w\right) \delta w d A=0$

Equation 78

The next step is taking the second and third last terms in Equation 78 and applying Green's theorem twice on each of these terms. However, for the first term application of the theorem 
must be done first with respect to $x$ and then $y$. The opposite sequence will be done for the second term. Thus for the first term, this process yields:

$$
\begin{aligned}
& \oiint\left((1-v) \frac{\partial^{2} w}{\partial x \partial y}\right) \frac{\partial^{2} \delta w}{\partial x \partial y} d A \\
& =\oint\left((1-v) \frac{\partial w}{\partial y}\right) \frac{\partial^{2} \delta w}{\partial x \partial y} n_{y} d s-\oiint\left((1-v) \frac{\partial w}{\partial y}\right) \frac{\partial^{3} \delta w}{\partial x^{2} \partial y} d A \\
& \oiint\left((1-v) \frac{\partial w}{\partial y}\right) \frac{\partial^{3} \delta w}{\partial x^{2} \partial y} d A \\
& =-\oint((1-v) w) \frac{\partial^{3} \delta w}{\partial y \partial x^{2}} n_{x} d s-\oiint((1-v) w) \frac{\partial^{4} \delta w}{\partial x^{2} \partial y^{2}} d A \\
& \oiint\left((1-v) \frac{\partial^{2} w}{\partial x \partial y}\right) \frac{\partial^{2} \delta w}{\partial x \partial y} d A=\oint\left((1-v) \frac{\partial w}{\partial y}\right) \frac{\partial^{2} \delta w}{\partial x \partial y} n_{y} d s \\
& +\oint((1-v) w) \frac{\partial^{3} \delta w}{\partial y \partial x^{2}} n_{x} d s+\oiint((1-v) w) \frac{\partial^{4} \delta w}{\partial x^{2} \partial y^{2}} d A
\end{aligned}
$$

Similarly, for the second term:

$$
\begin{aligned}
& \oiint\left((1-v) \frac{\partial^{2} w}{\partial x \partial y}\right) \frac{\partial^{2} \delta w}{\partial x \partial y} d A \\
& =-\oint\left((1-v) \frac{\partial w}{\partial x}\right) \frac{\partial^{2} \delta w}{\partial x \partial y} n_{x} d s-\oiint\left((1-v) \frac{\partial w}{\partial x}\right) \frac{\partial^{3} \delta w}{\partial y^{2} \partial x} d A \\
& \oiint\left((1-v) \frac{\partial w}{\partial x}\right) \frac{\partial^{3} \delta w}{\partial x \partial y^{2}} d A \\
& =\oint((1-v) w) \frac{\partial^{3} \delta w}{\partial x \partial y^{2}} n_{y} d s-\oiint((1-v) w) \frac{\partial^{4} \delta w}{\partial x^{2} \partial y^{2}} d A
\end{aligned}
$$




$$
\begin{aligned}
& \oiint\left((1-v) \frac{\partial^{2} w}{\partial x \partial y}\right) \frac{\partial^{2} \delta w}{\partial x \partial y} d A=-\oint\left((1-v) \frac{\partial w}{\partial x}\right) \frac{\partial^{2} \delta w}{\partial x \partial y} n_{x} d s \\
& -\oint((1-v) w) \frac{\partial^{3} \delta w}{\partial x \partial y^{2}} n_{y} d s+\oiint((1-v) w) \frac{\partial^{4} \delta w}{\partial x^{2} \partial y^{2}} d A
\end{aligned}
$$

Equation 84

Subsequently, Equation 78 takes the form:

$$
\begin{aligned}
& \oint\left(\frac{\partial w}{\partial x}\right) \frac{\partial^{2} \delta w}{\partial x^{2}} n_{y} d s-\oint(w) \frac{\partial^{3} \delta w}{\partial x^{3}} n_{y} d s+\oiint(w) \frac{\partial^{4} \delta w}{\partial x^{4}} d A \\
& -v \oint\left(\frac{\partial w}{\partial y}\right) \frac{\partial^{2} \delta w}{\partial x^{2}} n_{x} d s+v \oint(w) \frac{\partial^{3} \delta w}{\partial y \partial x^{2}} n_{x} d s+v \oiint(w) \frac{\partial^{4} \delta w}{\partial y^{2} \partial x^{2}} d A \\
& -\oint\left(\frac{\partial w}{\partial y}\right) \frac{\partial^{2} \delta w}{\partial y^{2}} n_{x} d s+\oint(w) \frac{\partial^{3} \delta w}{\partial y^{3}} n_{x} d s+\oiint(w) \frac{\partial^{4} \delta w}{\partial y^{4}} d A \\
& +v \oint\left(\frac{\partial w}{\partial x}\right) \frac{\partial^{2} \delta w}{\partial y^{2}} n_{y} d s-v \oint(w) \frac{\partial^{3} \delta w}{\partial x \partial y^{2}} n_{y} d s+v \oiint(w) \frac{\partial^{4} \delta w}{\partial x^{2} \partial y^{2}} d A \\
& +\oint\left((1-v) \frac{\partial w}{\partial y}\right) \frac{\partial^{2} \delta w}{\partial x \partial y} n_{y} d s+\oint((1-v) w) \frac{\partial^{3} \delta w}{\partial y \partial x^{2}} n_{x} d s \\
& +\oiint((1-v) w) \frac{\partial^{4} \delta w}{\partial x^{2} \partial y^{2}} d A-\oint\left((1-v) \frac{\partial w}{\partial x}\right) \frac{\partial^{2} \delta w}{\partial x \partial y} n_{x} d \\
& -\oint((1-v) w) \frac{\partial^{3} \delta w}{\partial x \partial y^{2}} n_{y} d s+\oiint((1-v) w) \frac{\partial^{4} \delta w}{\partial x^{2} \partial y^{2}} d A \\
& -\oiint\left(\frac{\omega^{2} \rho h w}{D}\right) \delta w d A=0
\end{aligned}
$$

Equation 85

Simplifying this equation gives:

$$
\begin{aligned}
& \oint\left(\frac{\partial w}{\partial x}\right)\left(\frac{\partial^{2} \delta w}{\partial x^{2}}+v \frac{\partial^{2} \delta w}{\partial y^{2}}\right) n_{y} d s-\oint\left(\frac{\partial w}{\partial y}\right)\left(\frac{\partial^{2} \delta w}{\partial y^{2}}+v \frac{\partial^{2} \delta w}{\partial x^{2}}\right) n_{x} d s \\
& +\oint\left((1-v) \frac{\partial w}{\partial y}\right) \frac{\partial^{2} \delta w}{\partial x \partial y} n_{y} d s-\oint\left((1-v) \frac{\partial w}{\partial x}\right) \frac{\partial^{2} \delta w}{\partial x \partial y} n_{x} d s \\
& -\oint(w)\left(\frac{\partial^{3} \delta w}{\partial x^{3}}+\frac{\partial^{3} \delta w}{\partial x \partial y^{2}}\right) n_{y} d s+\oint(w)\left(\frac{\partial^{3} \delta w}{\partial y^{3}}+\frac{\partial^{3} \delta w}{\partial y \partial x^{2}}\right) n_{x} d s \\
& +\oiint(w) \frac{\partial^{4} \delta w}{\partial x^{4}} d A+2 \oiint(w) \frac{\partial^{4} \delta w}{\partial y^{2} \partial x^{2}} d A+\oiint(w) \frac{\partial^{4} \delta w}{\partial y^{4}} d A \\
& -\oiint\left(\frac{\omega^{2} \rho h w}{D}\right) \delta w d A=0
\end{aligned}
$$

Equation 86 
It is noteworthy that the first two integrals in Equation 86, respectively, are representation of virtual bending moments $\delta M_{x}$ and $\delta M_{y}$, written in terms of the virtual displacement $\delta w$. Similarly, the next two integrals represent virtual twisting moment $\delta M_{x y}$. Integrals five and six are representation of virtual shear forces $\delta Q_{x}$ and $\delta Q_{y}$, respectively. Hence, one can rewrite Equation 86 as:

$$
\begin{aligned}
& -\oint\left(\frac{\partial w}{\partial x}\right)\left(\delta M_{x}\right) n_{y} d s+\oint\left(\frac{\partial w}{\partial y}\right)\left(\delta M_{y}\right) n_{x} d s \\
& -\oint\left(\frac{\partial w}{\partial y}\left(\delta M_{x y}\right)\right) n_{y} d s+\oint\left(\frac{\partial w}{\partial x}\left(\delta M_{x y}\right)\right) n_{x} d s \\
& +\oint(w)\left(\delta Q_{y}\right) n_{y} d s-\oint(w)\left(\delta Q_{x}\right) n_{x} d s \\
& +\oint(w)\left(\frac{\partial^{4} \delta w}{\partial x^{4}}+2 \frac{\partial^{4} \delta w}{\partial y^{2} \partial x^{2}}+\frac{\partial^{4} \delta w}{\partial y^{4}}-\omega^{2} \rho h \delta w\right) d A=0
\end{aligned}
$$

Equation 87

Unlike the reduction done for FEM development where work of virtual boundary integrals were equal to zero, virtual boundary integrals do not yield zero in this equation. This is becaue virtual loads can assume finite small values under any boundary condition of a free vibrating plate.

The area integral in this equation represents the governing equation of the plate vibration in terms of the weighting function $\delta w$. Because the basis functions used for development of the dynamic finite plate element are solutions of the governing differential equation and the same functions are used for virtual displacement in Galerkin method, the area integrals will vanish from Equation 86 and Equation 87 and DFE will involve only line integrals.

Equation 86 is the fundamental equation used for deriving the dynamic finite element formulation. Similar procedure to Equation 32 through Equation 36 (see chapter 3) are followed 
using basis functions of Equation 72 to generate frequency dependent shape function vector $\left[N_{\omega}(x, y)\right]$.

Although as shown in Figure 5, these functions are dependent on vibrational frequency $\omega$ through $\gamma_{x y}=\left(\frac{\rho h \omega^{2}}{D}\right)^{\frac{1}{4}}$, they follow the general properties of the regular shape functions such that their value is one at their corresponding nodes while all other nodal values are zero over every frequency. These shape functions are reported and discussed further in APPENDIX A.

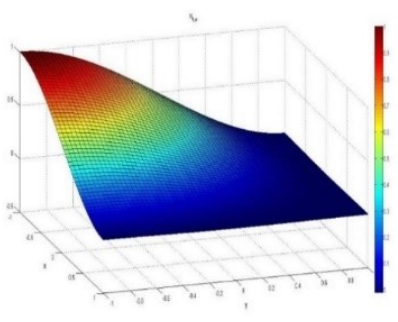

(a)

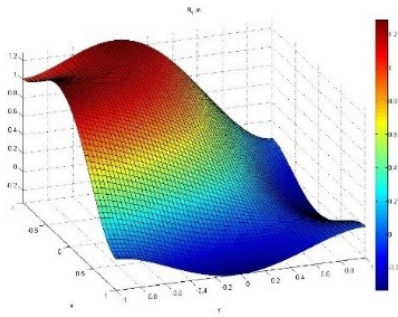

(b)

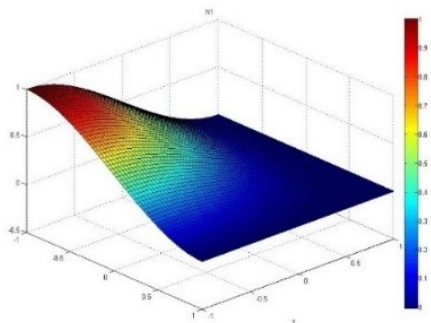

(c)

Figure 5 Graph of DFE shape function $N_{1, \omega}$ for value of $\gamma_{x y}=10 e-6$ (a) and for value of $\gamma_{x y}=2$ (b) and first Hermite shape function (c) over the plate element of Figure 4

By substituting the shape function vector $\left[N_{\omega}(x, y)\right]$ in Equation 86 , the resulting dynamic finite element matrix, $\left[K_{\omega}\right]$, is generated as a summation of six frequency dependent submatrices. The positive sense of integration must be considered counter clockwise around the element boundary in accordance with Green's theorem. 


$$
\begin{aligned}
& {\left[K_{\omega}\right]=D\left[\left.\int_{-1}^{1}\left(\frac{\partial\left[N_{\omega}^{T}\right]}{\partial x}\right)\left(\frac{\partial^{2}\left[\delta N_{\omega}\right]}{\partial x^{2}}+v \frac{\partial^{2}\left[\delta N_{\omega}\right]}{\partial y^{2}}\right)\right|_{x=-1} ^{x=1} d y\right.} \\
& -\left.\int_{-1}^{1}\left(\frac{\partial\left[N_{\omega}^{T}\right]}{\partial y}\right)\left(\frac{\partial^{2}\left[\delta N_{\omega}\right]}{\partial y^{2}}+v \frac{\partial^{2}\left[\delta N_{\omega}\right]}{\partial x^{2}}\right)\right|_{y=1} ^{y=-1} d x \\
& \left.+\int_{-1}^{1}\left((1-v) \frac{\partial\left[N_{\omega}^{T}\right]}{\partial y}\right) \frac{\partial^{2}\left[\delta N_{\omega}\right]}{\partial x \partial y}\right]_{x=-1}^{x=1} d y \\
& \left.-\int_{-1}^{1}\left((1-v) \frac{\partial\left[N_{\omega}^{T}\right]}{\partial x}\right) \frac{\partial^{2}\left[\delta N_{\omega}\right]}{\partial x \partial y}\right]_{y=1}^{y=-1} d x \\
& -\left.\int_{-1}^{1}\left(\left[N_{\omega}^{T}\right]\right)\left(\frac{\partial^{3}\left[\delta N_{\omega}\right]}{\partial x^{3}}+\frac{\partial^{3}\left[\delta N_{\omega}\right]}{\partial x \partial y^{2}}\right)\right|_{x=-1} ^{x=1} d y \\
& \left.+\left.\int_{-1}^{1}\left(\left[N_{\omega}^{T}\right]\right)\left(\frac{\partial^{3}\left[\delta N_{\omega}\right]}{\partial y^{3}}+\frac{\partial^{3}\left[\delta N_{\omega}\right]}{\partial y \partial x^{2}}\right)\right|_{y=1} ^{y=-1} d x\right]=0
\end{aligned}
$$

Equation 88

Therefore, the dynamic stiffness matrix outlined in Equation 88 will also depends on vibrational frequency. Although unlike Galekin method's form used for FEM in Equation 53 (see chapter 3), DFE integrals do not include symmetry exclusively, the use of solutions of the governing differential equation guarantees symmetry of the final DFE matrix obtained implicitly.

Much like the finite element analysis, by constraining the appropriate nodal degrees of freedom and finding the system's Eigenvalues one can find the natural frequencies corresponding to a set of boundary condition. Note that, however, the Eigenproblem generated through dynamic finite element is non-linear in $\omega$ and results a transcendental equation with multiple possible solutions for natural frequency even using one element.

This is a major advantage of using dynamic finite element over finite element analysis. The Eigenproblem resulting from the finite element analysis is linear in nature and produces a polynomial in variable $\omega$. Because a polynomial can generate maximum number of roots equal 
to its degree, FEM vibrational analysis leads to finite number of answers, and finding higher natural frequency generally requires mesh refinement. 


\section{NUMERICAL EVALUATION}

In this chapter, the 12-DoF dynamic finite element derived and presented in chapter 5 is used to model free vibrational behaviour of a square plate under four boundary conditions for which analytical results are available in open literature. The performance of DFE is compared with the 12-DoF Hermitian plate elements developed in chapter 3, as well as analytical frequency data reported by Leissa [4].

As mentioned in the previous chapter, the dynamic finite element produces a nonlinear Eigenvalue problem of the following form:

$\left[\mathrm{K}_{\omega}\right][w]=0$

Equation 89

creating a transcendental equation in variable $\omega$ with multiple roots. However, the conventional finite element analysis requires solving the linear Eigenvalue problem of the system, written as:

$\left([\mathrm{K}]-\omega^{2}[m]\right)[w]=0$

Equation 90

which produces a polynomial with finite number of roots.

Consider a two-unit by two-unit square plate element as shown in Figure 6, to be simply supported on 3 edges and free on one edge (SS-SS-SS-F boundary condition) with Poisson ratio of 0.3 . In order to eliminate material dependency, vibrational analysis is done by normalizing the results in terms of $\omega \sqrt{\rho h / D}$. 


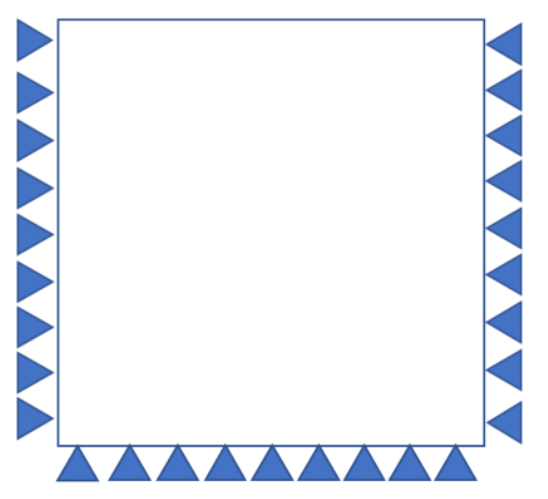

Figure 6 Square plate with two unit side length under SS-SS-SS-F boundary condition

The free vibrational analysis of this system subject to SS-SS-SS-F boundary conditions is carried out, where the end conditions are enforced by eliminating rows and columns of the stiffness matrices corresponding to the restricted degrees of freedom, and results in a 2 by 2 Eigenvalue problem. The DFE Eigenproblem has infinite number of solutions, which can be detected by visual inspection of the graph representing the determinant of the system's dynamic stiffness matrix, $\left[\mathrm{K}_{\omega}\right]$, as shown in Figure 7 , or through the application of Wittrick-Williams (W-W) algorithm [38]. The system's first five natural frequencies obtained through the DFE vibrational analysis for this set of boundary conditions are compared with analytical solutions reported by Leissa [4], obtained using double Fourier series, as well as those obtained from the conventional FEM (see Table 1). 


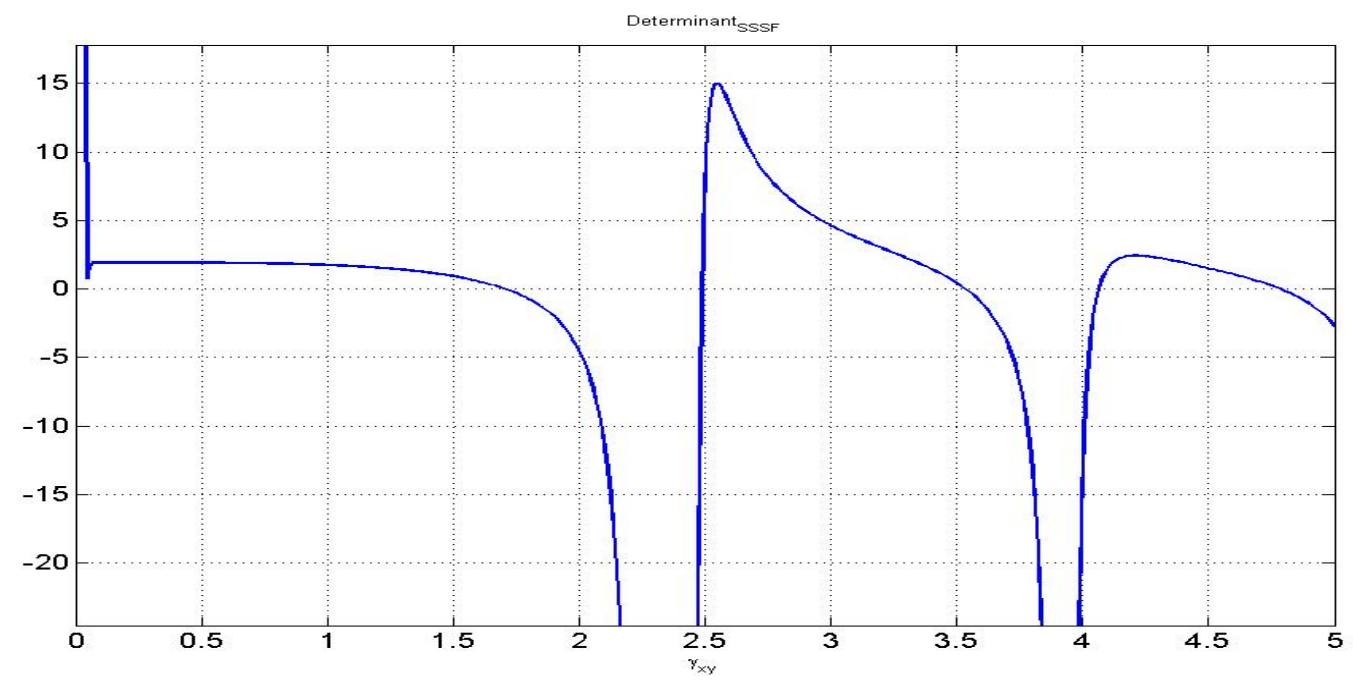

Figure 7 Plot of transcendental DFE determinant function for SS-SS-SS-F boundary condition against $\gamma_{x y}=\left(\frac{\rho h \omega^{2}}{D}\right)^{\frac{1}{4}}$

Table 1 Comparison of 1 element DFE formulation and analytical solution for Values of $\omega \sqrt{\rho h / D}$

\begin{tabular}{|c|c|c|c|}
\hline $\begin{array}{c}\text { Mode } \\
\text { Number }\end{array}$ & $\begin{array}{c}\text { Analytical } \\
\text { Result [4] }\end{array}$ & $\begin{array}{c}\text { 12 DoF DFE } \\
\text { 1 element mesh }\end{array}$ & $\begin{array}{c}\text { 12 DoF FEM } \\
\text { 1 element mesh }\end{array}$ \\
\hline 1.00 & 2.92 & 2.91 & 3.18 \\
\hline 2.00 & 6.94 & 6.23 & 12.98 \\
\hline 3.00 & 10.30 & 12.51 & - \\
\hline 4.00 & 14.77 & 16.52 & - \\
\hline 5.00 & 15.47 & 22.90 & - \\
\hline
\end{tabular}

As can be seen from Table 1, DFE results obtained using a single-DFE model are in good agreement with the analytical solutions. This is especially notable for the fundamental natural frequency, where a single DFE produces near analytical result, i.e., $0.3 \%$ difference. As expected, as the mode number increases, the difference between the DFE and analytical data increases, to $10 \%, 21 \%, 11 \%$, and $48 \%$, for the 2 nd through 5 th frequencies, respectively. On the other hand, the single-element conventional FEM model can only capture the first two frequencies, with over $9 \%$ and $109 \%$ differences with the analytical data for the 1 st and 2 nd modes, respectively. 
Additionally, DFE matrices and shape functions contain a common denominator. The zeros of this denominator are called poles, which cause the determinant matrix to approach infinity and correspond to all edges fixed boundary condition. For a single FEM element, fixing all edges to zero results in removal of all rows and columns of stiffness and mass matrices, and therefore, capturing the modes of this boundary condition is not possible. However, the DFE element has the potential to detect vibration modes of this boundary condition using its poles.

The same analysis was performed for fundamental natural frequencies of three other sets of boundary conditions and a comparison with the FEM plate is reported in Table 2 .

Table 2 Comparison of fundamental natural frequency obtained from DFE and FEM element for Values of $\omega \sqrt{\rho h / D}$

\begin{tabular}{|c|c|c|c|}
\hline $\begin{array}{l}\text { Boundary } \\
\text { Condition }\end{array}$ & $\begin{array}{c}\text { Analytical } \\
\text { Result [4] }\end{array}$ & 12 DoF DFE & 12 DoF FEM \\
\hline C-F-SS-F & 3.79 & 3.81 & 5.13 \\
\hline C-C-F-F & 1.74 & 1.82 & 1.92 \\
\hline SS-F-SS-F & 2.40 & 2.42 & 2.74 \\
\hline
\end{tabular}

It is evident that due to inclusion of dynamic effects, i.e., frequency-dependency, in element formulation, a single DFE plate has significantly improved performance over its FEM counterpart, as the element can capture natural frequencies with higher accuracy and is able to detect significantly higher number of modes.

\subsection{CONVERGENCE STUDIES}

To further evaluate the proposed dynamic finite element performance, a convergence study for the modes two through five, over a two by two plate, under three edges simply supported and 
one edge fixed boundary condition (SS-SS-SS-F), and Poisson's ratio of 0.3 was performed. A comparative evaluation of these modes using 12-DoF Hermitian FEM was also carried out. Mesh size was increased from a two by two grid (four elements) to a grid of five by five size (25 elements) and Eigenvalues were recorded for both formulations.

The results are reported in terms of $\omega \sqrt{\rho h / D}$ so that material dependency is not present. The errors of both analyses are calculated with respect to results from Leissa [4] and the convergence graphs, representing the error vs. the number of elements are presented in the following Figures and Tables.

As shown, both formulations have comparable performance on convergence to the analytical solutions and reach less than five percent error using a 25-element mesh. This comparable performance is achieved by faster convergence of a formulation with higher initial error. For example, for the second mode of vibration while FEM formulation has larger error over the two by two mesh compared to DFE formulation, it has faster convergence towards the analytical solution, yielding comparable performance. The opposite is observed for the fifth mode of vibration.

It is worth mentioning that both DFE and FEM plates in this research are $12 \mathrm{DoF}$ elements and apply similar degradation to plate problem. For example, both elements cannot capture the curvature $\frac{\partial^{4} w}{\partial^{2} x \partial^{2} y}$ and cannot enforce the inter-element shear stress continuity. These two factors are possible reasons for similar convergence characteristics of the two formulations. 
Table 3 Second mode convergence analysis of DFE and FEM elements for $\omega \sqrt{\rho h / D}$ on a SS-SS-SS-F plate

\begin{tabular}{|l|l|l|l|l|l|l|l|l|}
\hline Result & \%Error & Result & \%Error & Result & \%Error & Result & \%Error & Mode 2 \\
\hline $2 \times 2$ DFE & $2 \times 2$ DFE & $3 \times 3$ DFE & $3 \times 3$ DFE & $4 \times 4$ DFE & $4 \times 4$ DFE & $5 \times 5$ DFE & $5 \times 5$ DFE & Analytical \\
\hline 6.54 & -5.76 & 6.65 & -4.18 & 6.75 & -2.74 & 6.81 & -1.87 & \\
$2 \times 2$ FEM & $2 \times 2$ FEM & $3 \times 3$ FEM & $3 \times 3$ FEM & $4 \times 4$ FEM & $4 \times 4$ FEM & $5 \times 5$ FEM & $5 \times 5$ FEM & \multirow{2}{*}{6.94} \\
\cline { 1 - 6 } 6.47 & -6.77 & 6.65 & -4.18 & 6.75 & -2.74 & 6.81 & -1.87 & \\
\hline
\end{tabular}

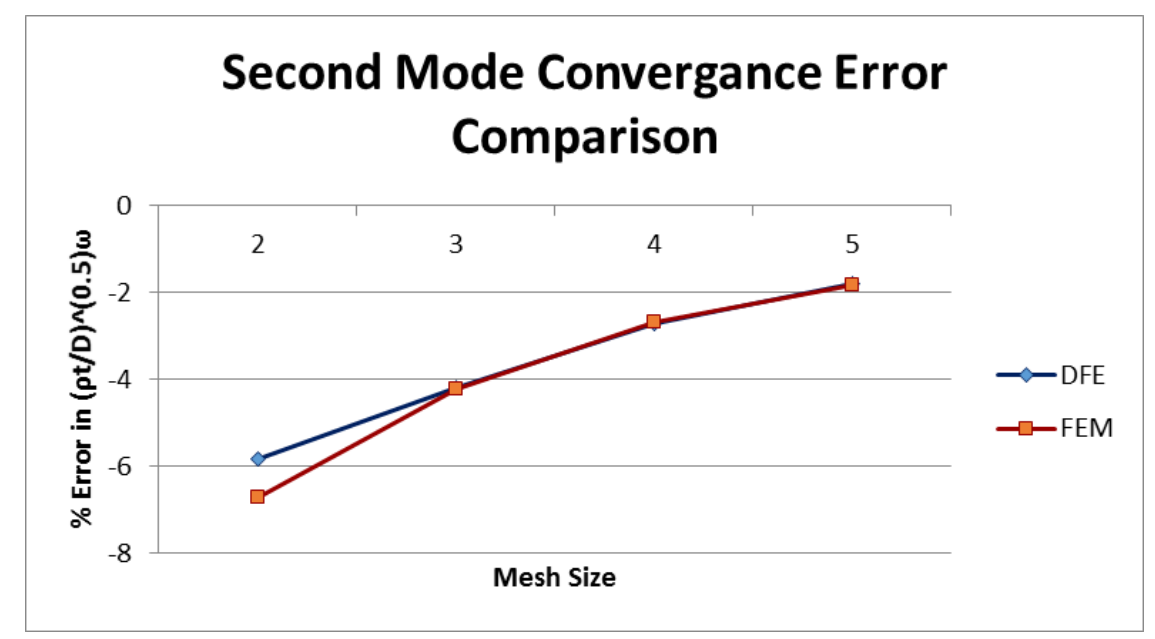

Figure 8 Comparison of convergence of $\omega \sqrt{\rho h / D}$ values between FEM and DFE models for second mode of vibration of a plate under SS-SS-SS-F boundary condition

Table 4 Third mode convergence analysis of DFE and FEM elements for $\omega \sqrt{\rho h / D}$ on a SS-SS-SS-F plate

\begin{tabular}{|c|c|c|c|c|c|c|c|c|}
\hline Result & \%Error & Result & \%Error & Result & \%Error & Result & \%Error & Mode 3 \\
\hline $2 \times 2$ DFE & $2 \times 2$ DFE & $3 \times 3$ DFE & $3 \times 3$ DFE & 4x4 DFE & $4 \times 4$ DFE & $5 \times 5$ DFE & $5 \times 5$ DFE & Analytical \\
\hline 10.07 & -2.23 & 10.23 & -0.68 & 10.32 & 0.19 & 10.32 & 0.19 & \multirow{3}{*}{10.3} \\
\hline $2 \times 2$ FEM & $2 \times 2$ FEM & $3 \times 3$ FEM & $3 \times 3$ FEM & $4 \times 4$ FEM & $4 \times 4$ FEM & $5 \times 5$ FEM & $5 \times 5$ FEM & \\
\hline 11.36 & 10.29 & 10.38 & 0.78 & 10.36 & 0.58 & 10.35 & 0.49 & \\
\hline
\end{tabular}




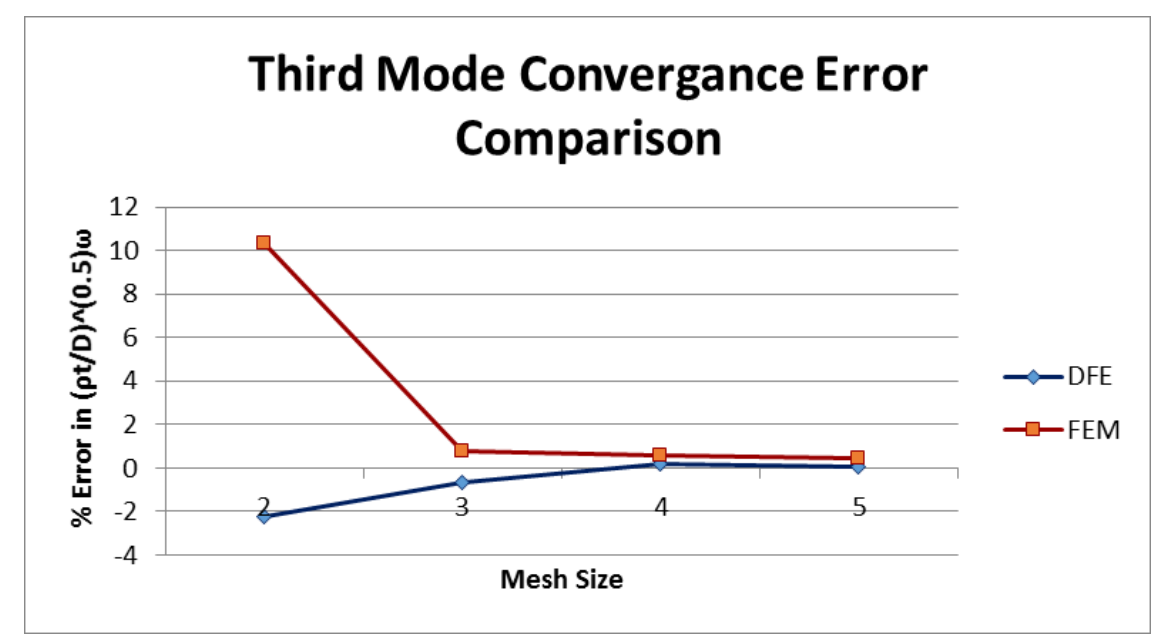

Figure 9 Comparison of convergence of $\omega \sqrt{\rho h / D}$ values between FEM and DFE models for third mode of vibration of a plate under SSSS-SS-F boundary condition

Table 5 Fourth mode convergence analysis of DFE and FEM elements for $\omega \sqrt{\rho h / D}$ on a SS-SS-SS-F plate

\begin{tabular}{|l|l|l|l|l|l|l|l|l|}
\hline Result & \%Error & Result & \%Error & Result & \%Error & Result & \%Error & Mode 4 \\
\hline $2 \times 2$ DFE & $2 \times 2$ DFE & $3 \times 3$ DFE & $3 \times 3$ DFE & $4 \times 4$ DFE & $4 \times 4$ DFE & $5 \times 5$ DFE & $5 \times 5$ DFE & Analytical \\
\hline 15.24 & 3.18 & 13.83 & -6.36 & 14.08 & -4.67 & 14.28 & -3.32 & \\
\cline { 1 - 6 } $2 \times 2$ FEM & $2 \times 2$ FEM & $3 \times 3$ FEM & $3 \times 3$ FEM & $4 \times 4$ FEM & $4 \times 4$ FEM & $5 \times 5$ FEM & $5 \times 5$ FEM & \multirow{2}{*}{14.77} \\
\cline { 1 - 6 } 15.20 & 2.91 & 13.83 & -6.36 & 14.10 & -4.54 & 14.30 & -3.18 & \\
\hline
\end{tabular}

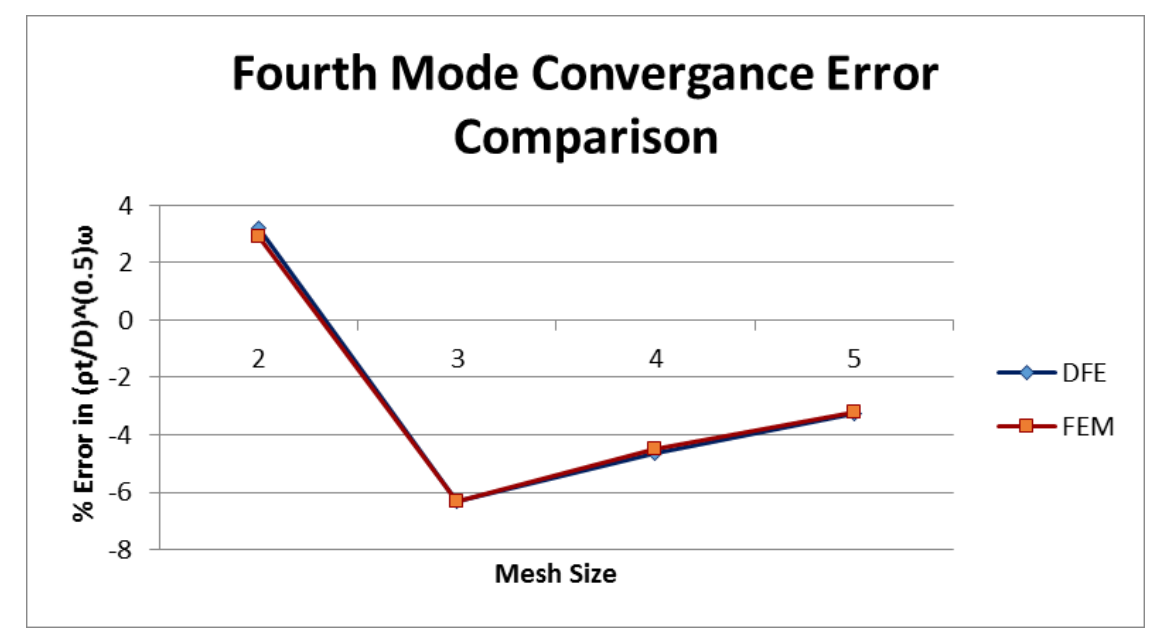

Figure 10 Comparison of convergence of $\omega \sqrt{\rho h / D}$ values between FEM and DFE models for fourth mode of vibration of a plate under SS-SS-SS-F boundary condition 
Table 6 Fifth mode convergence analysis of DFE and FEM elements for $\omega \sqrt{\rho h / D}$ on a SS-SS-SS-F plate

\begin{tabular}{|c|c|c|c|c|c|c|c|c|}
\hline Result & \%Error & Result & \%Error & Result & \%Error & Result & \%Error & Mode 5 \\
\hline $2 \times 2$ DFE & $2 \times 2$ DFE & $3 \times 3$ DFE & $3 \times 3$ DFE & 4x4 DFE & 4x4 DFE & $5 \times 5$ DFE & $5 \times 5$ DFE & Analytical \\
\hline 16.42 & 6.14 & 14.61 & -5.56 & 14.86 & -3.94 & 15.04 & -2.78 & \multirow{3}{*}{15.47} \\
\hline $2 \times 2$ FEM & $2 \times 2$ FEM & $3 \times 3$ FEM & $3 \times 3$ FEM & $4 \times 4$ FEM & 4x4 FEM & $5 \times 5$ FEM & $5 \times 5$ FEM & \\
\hline 16.07 & 3.88 & 14.69 & -5.04 & 14.93 & -3.49 & 15.07 & -2.59 & \\
\hline
\end{tabular}

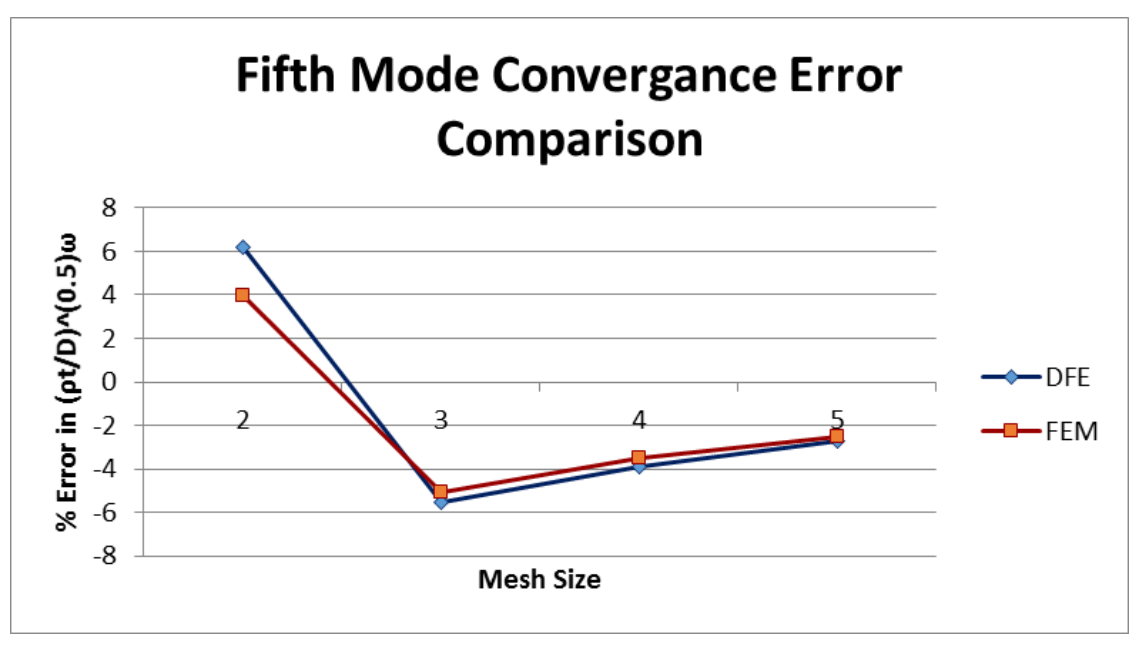

Figure 11 Comparison of convergence of $\omega \sqrt{\rho h / D}$ values between FEM and DFE models for fifth mode of vibration of a plate under SSSS-SS-F boundary condition 


\section{CONCLUDING REMARKS}

In this research, a 12-DoF frequency-dependent formulation for two-dimensional elements, based on dynamic finite element method is presented. The DFE method relies on using frequency dependent shape functions in contrast to polynomials used by FEM method.

Literature survey on DFE has shown this technique to be very powerful method in case of beams, producing exact results with one element in case of uncoupled beams, and having very fast convergence rate for beams with couplings. This served as a motivation for the present work, and the method is researched here for its applicability to two-dimensional thin plate elements.

The governing differential equation of thin plates is a two-dimensional partial differential equation, which requires an infinite dimensional solution set such as those produced by double Fourier series. This property is contrary to beam DFE elements, which are defined by an ordinary differential equation, with finite number of solutions that can be fully incorporated in a finite element matrix.

Although previous formulations, with complete infinite dimensional solution space have been used, the applicability of such developments is limited to specific geometric, loadings and boundary condition.

The DFE element developed here utilized a finite subset of the complete solution space of the plate problem. Three different solution sets are developed and analyzed for the plate based on distribution of dynamic force over element partial curvatures. One solution set did not produce minimum number of the required independent basis function and the second set proved to be divergent. 
The third set reduces the plate equation to two independent beams. These beam-like solutions to the governing differential equation of the Kirchhoff plate, are then rearranged as basis functions of the approximation space, to produce a linearly independent set of shape functions, for a 4node plate with 12 DoFs. This arrangement was done such that the element formulation will converge to Hermitian FEM, for limiting case of zero vibrational frequency and depart for the non-zero values.

The DFE plate showed good agreement with analytical solutions for one element, and is capable of producing multiple natural frequencies using a single element, specially for lower frequency modes. The convergence studies performed showed comparable performance between DFE and FEM elements.

Despite comparable convergence performance and complexity of the Eigenvalue problem, DFE formulations are still considered superior to their FEM counterparts, particularly for the ability to capture infinite number of vibrational modes with comparable mesh size. Also, the DFE methods developed can be readily extended to quadrilateral and other geometries (see APPENDIX B) without loss of accuracy, through exact line integrations, which eliminates the need of using Jacobian determinants and numerical evaluation.

The amount of research done on dynamic formulation of plate elements shows a clear room for improvement. For future, the author recommends considering other solution sets of the governing differential equation.

These solutions should be analyzed in terms of the distribution of the dynamic force over element strains, and grouped to produce independent shape functions. Also, combining various solution sets with appropriate coefficients can be done to reduce the number of basis functions to 
that applicable to a plate element. These solution enrichments can provide higher order DFE such as $16-$ DoF plate elements. Also, multiple element types can be obtained with same order, each tailored for a specific problem, based on specific restriction of the governing differential equation.

Future researchers may also consider extending the DFE formulation to thick plates, laminated plates, composite plates, plates with varying mass, plates with cut-outs and plates with in-plane loading. 


\section{APPENDIX A}

In this appendix, a report of shape functions obtained from DFE formulation, mentioned in chapter 5, for a $2 \times 2$ plate element depicted in chapter 3-Figure 3, and their graphs evaluated over the plate domain is presented. A comparison is made between the DFE shape functions (shape functions with subscript $\omega$ ), and Hermitian shape functions of chapter 3 FEM plate, obtained from polynomial distribution of the displacement function [56].

While the DFE shape functions are dependent on the vibrational frequency, through the variable $\gamma_{x y}=\left(\frac{\rho h \omega^{2}}{D}\right)^{\frac{1}{4}}$ over the plate domain, it is noted that even by having complex equations, these shape functions and their derivatives converge to their Hermitian counterparts, for small values of vibrational frequency, and thus allow the DFE to be used successfully as an FEM equivalent analysis tool, for static structural problems as well.

It is also evident that much like the Hermite shape functions, DFE shape functions maintain the symmetry and rotation properties of the nodes. This property is also held true for shape function derivatives, and is observed for all values of vibrational frequency.

Although the DFE shape functions are dependent on the vibrational frequency, they have a value of one at their respective nodes, and are equal to zero at the remaining element nodes regardless of the vibrational frequency, which is a critical criterion for error minimization in Galerkin's residual scheme. For the shape functions $N_{1, \omega}, N_{4, \omega}, N_{7, \omega}$ and $N_{10, \omega}$ this requirement is met by the shape functions themselves. For $N_{2, \omega}, N_{5, \omega}, N_{8, \omega}$ and $N_{11, \omega}$ their partial derivative

with respect to $x$ holds this property as these shape functions are related to the nodal slope $\frac{\partial w}{\partial x}$. Similarly, for $N_{3, \omega}, N_{6, \omega}, N_{9, \omega}$ and $N_{12, \omega}$ partial derivatives with respect to the variable $y$ satisfy this property. 


$$
\begin{aligned}
& N_{1, \omega}=\frac{\left(\sin \left(\gamma_{x y}\right)+\sinh \left(\gamma_{x y}\right)\right)\left(\cos \left(\gamma_{x y} x\right)+\cos \left(\gamma_{x y} y\right)\right)}{8\left(\cos \left(\gamma_{x y}\right) \sinh \left(\gamma_{x y}\right)+\cosh \left(\gamma_{x y}\right) \sin \left(\gamma_{x y}\right)\right)}-\frac{\sin \left(\gamma_{x y} y\right)-\sinh \left(\gamma_{x y} y\right)}{4\left(\sin \left(\gamma_{x y}\right)-\sinh \left(\gamma_{x y}\right)-\gamma_{x y} \cos \left(\gamma_{x y}\right)+\gamma_{x y} \cosh \left(\gamma_{x y}\right)\right)} \\
& -\frac{\sin \left(\gamma_{x y}\right)\left(\cos \left(\gamma_{x y} x\right)-\cosh \left(\gamma_{x y} x\right)\right)}{8\left(\cos \left(\gamma_{x y}\right) \sinh \left(\gamma_{x y}\right)+\cosh \left(\gamma_{x y}\right) \sin \left(\gamma_{x y}\right)\right)}-\frac{\left.\sin \left(\gamma_{x y} y\right)-\cosh \left(\gamma_{x y} y\right)\right)}{8\left(\cos \left(\gamma_{x y}\right) \sinh \left(\gamma_{x y}\right)+\cosh \left(\gamma_{x y}\right) \sin \left(\gamma_{x y}\right)\right)} \\
& -\frac{\sin \left(\gamma_{x y} x\right)-\sinh \left(\gamma_{x y} x\right)}{4\left(\sin \left(\gamma_{x y}\right)-\sinh \left(\gamma_{x y}\right)-\gamma_{x y} \cos \left(\gamma_{x y}\right)+\gamma_{x y} \cosh \left(\gamma_{x y}\right)\right)} \\
& +\frac{\left(x \sin \left(\gamma_{x y} y\right)+y \sin \left(\gamma_{x y} x\right)\right)\left(\sin \left(\gamma_{x y}\right)-\sinh \left(\gamma_{x y}\right)+\gamma_{x y} \cos \left(\gamma_{x y}\right)-\gamma_{x y} \cosh \left(\gamma_{x y}\right)\right)}{8 \gamma_{x y}\left(\cos \left(\gamma_{x y}\right) \sinh \left(\gamma_{x y}\right)-\cosh \left(\gamma_{x y}\right) \sin \left(\gamma_{x y}\right)\right)} \\
& -\frac{y\left(\sin \left(\gamma_{x y} x\right)-\sinh \left(\gamma_{x y} x\right)\right)\left(\sin \left(\gamma_{x y}\right)+\gamma_{x y} \cos \left(\gamma_{x y}\right)\right)}{8 \gamma_{x y}\left(\cos \left(\gamma_{x y}\right) \sinh \left(\gamma_{x y}\right)-\cosh \left(\gamma_{x y}\right) \sin \left(\gamma_{x y}\right)\right)}-\frac{x y y}{8 \gamma_{x y}\left(\cos \left(\gamma_{x y}\right) \sinh \left(\gamma_{x y}\right)-\cosh \left(\gamma_{x y}\right) \sin \left(\gamma_{x y}\right)\right)} \\
& -\frac{\gamma_{x y} y\left(\cos \left(\gamma_{x y} x\right)-\cosh \left(\gamma_{x y} x\right)\right)\left(\sin \left(2 \gamma_{x y}\right)-2 \cosh \left(\gamma_{x y}\right) \sin \left(\gamma_{x y}\right)\right)}{8\left(\cos \left(\gamma_{x y}\right) \sinh \left(\gamma_{x y}\right)+\cosh \left(\gamma_{x y}\right) \sin \left(\gamma_{x y}\right)\right)\left(\sin \left(\gamma_{x y}\right)-\sinh \left(\gamma_{x y}\right)-\gamma_{x y} \cos \left(\gamma_{x y}\right)+\gamma_{x y} \cosh \left(\gamma_{x y}\right)\right)} \\
& +\frac{\gamma_{x y} x \cos \left(\gamma_{x y} y\right)\left(\sin \left(\gamma_{x y}\right)+\sinh \left(\gamma_{x y}\right)\right)\left(\cos \left(\gamma_{x y}\right)-\cosh \left(\gamma_{x y}\right)\right)}{4\left(\cos \left(\gamma_{x y}\right) \sinh \left(\gamma_{x y}\right)+\cosh \left(\gamma_{x y}\right) \sin \left(\gamma_{x y}\right)\right)\left(\sin \left(\gamma_{x y}\right)-\sinh \left(\gamma_{x y}\right)-\gamma_{x y} \cos \left(\gamma_{x y}\right)+\gamma_{x y} \cosh \left(\gamma_{x y}\right)\right)} \\
& +\frac{\gamma_{x y} y \cos \left(\gamma_{x y} x\right)\left(\sin \left(\gamma_{x y}\right)+\sinh \left(\gamma_{x y}\right)\right)\left(\cos \left(\gamma_{x y}\right)-\cosh \left(\gamma_{x y}\right)\right)}{4\left(\cos \left(\gamma_{x y}\right) \sinh \left(\gamma_{x y}\right)+\cosh \left(\gamma_{x y}\right) \sin \left(\gamma_{x y}\right)\right)\left(\sin \left(\gamma_{x y}\right)-\sinh \left(\gamma_{x y}\right)-\gamma_{x y} \cos \left(\gamma_{x y}\right)+\gamma_{x y} \cosh \left(\gamma_{x y}\right)\right)} \\
& -\frac{\gamma_{x y} x \sin \left(\gamma_{x y}\right)\left(\cos \left(\gamma_{x y} y\right)-\cosh \left(\gamma_{x y} y\right)\right)\left(\cos \left(\gamma_{x y}\right)-\cosh \left(\gamma_{x y}\right)\right)}{4\left(\cos \left(\gamma_{x y}\right) \sinh \left(\gamma_{x y}\right)+\cosh \left(\gamma_{x y}\right) \sin \left(\gamma_{x y}\right)\right)\left(\sin \left(\gamma_{x y}\right)-\sinh \left(\gamma_{x y}\right)-\gamma_{x y} \cos \left(\gamma_{x y}\right)+\gamma_{x y} \cosh \left(\gamma_{x y}\right)\right)}
\end{aligned}
$$




$$
N_{1}=-\frac{(x-1)(y-1)\left(x^{2}+x+y^{2}+y-2\right)}{8}
$$

A- 2

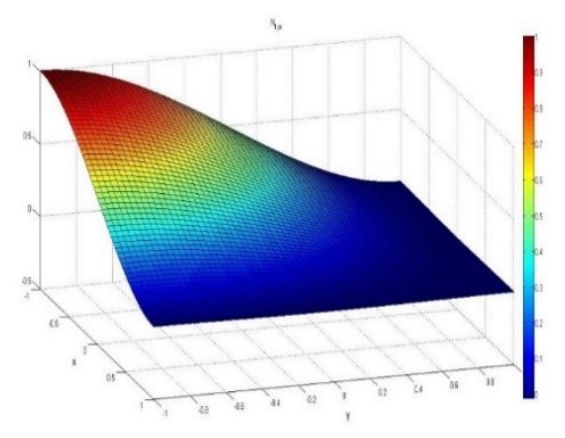

(a)

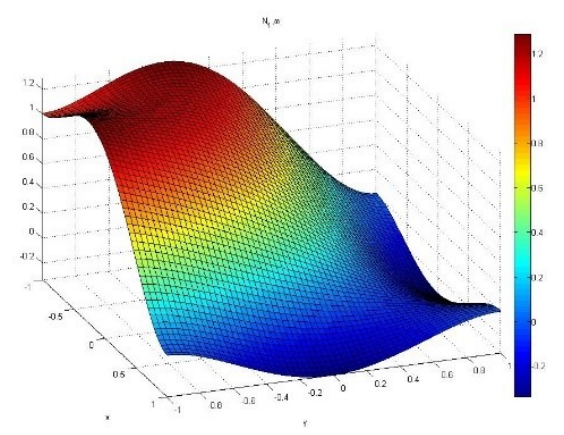

(b)

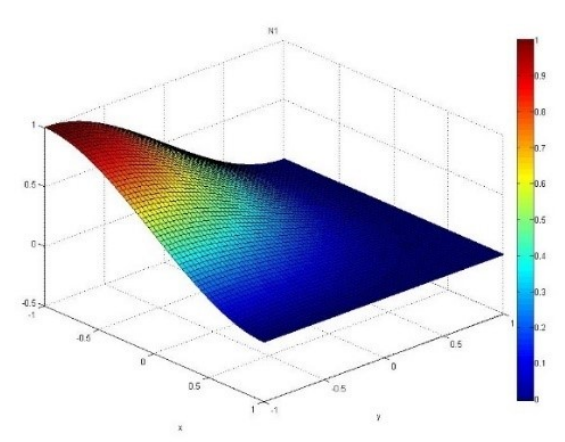

(c)

Figure 12 Graph of DFE shape function $N_{1, \omega}$ for value of $\gamma_{x y}=10 e-6$ (a) and for value of $\gamma_{x y}=2$ (b) and first Hermite shape function (c) over the plate element of Figure 3 


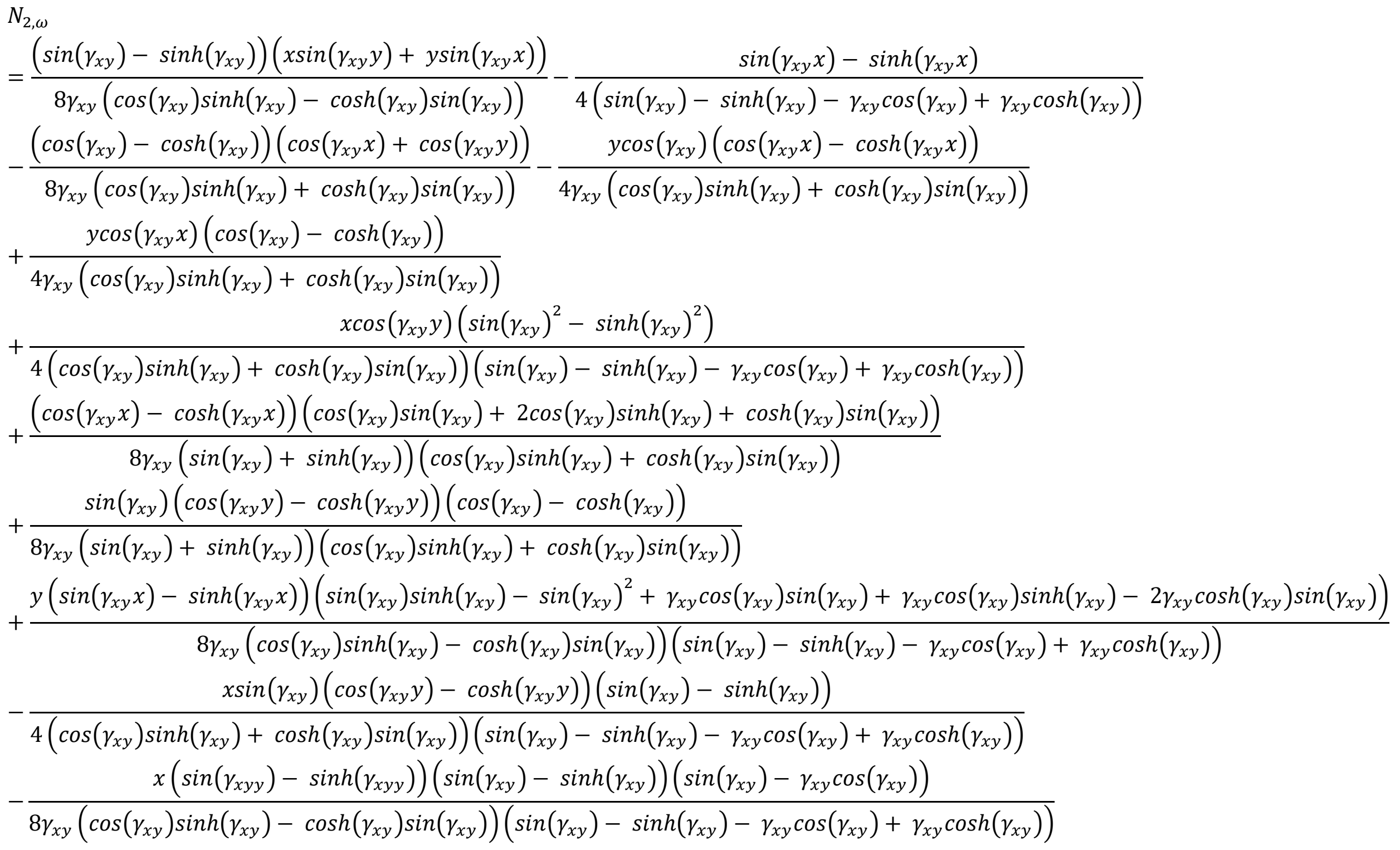




$$
-1-
$$




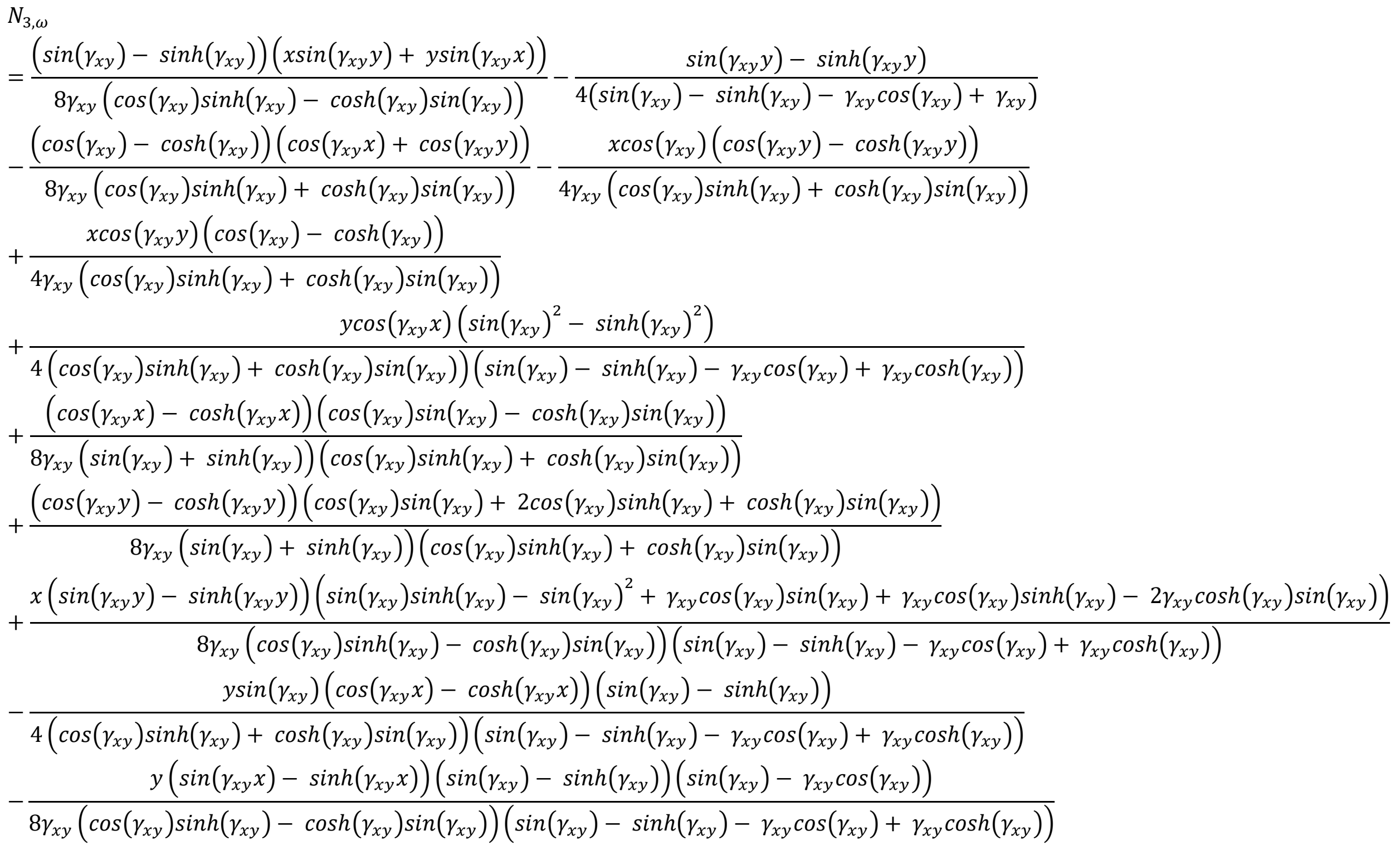




$$
N_{3}=-\frac{(x-1)(y-1)^{2}(y+1)}{8}
$$

A- 6

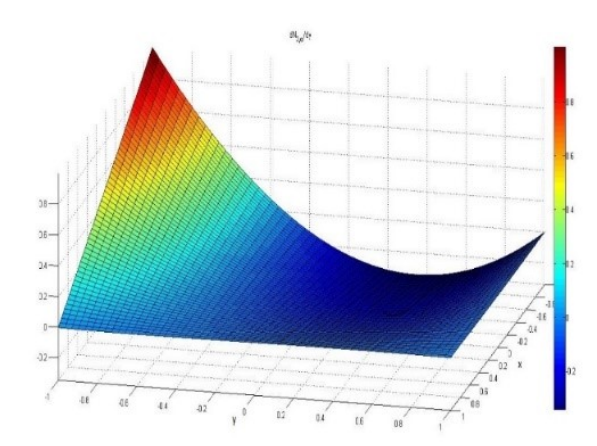

(a)

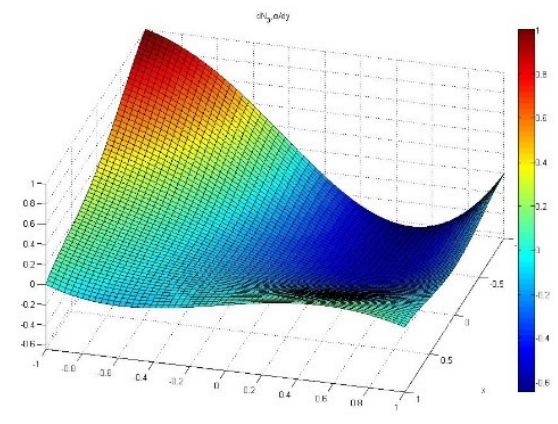

(b)

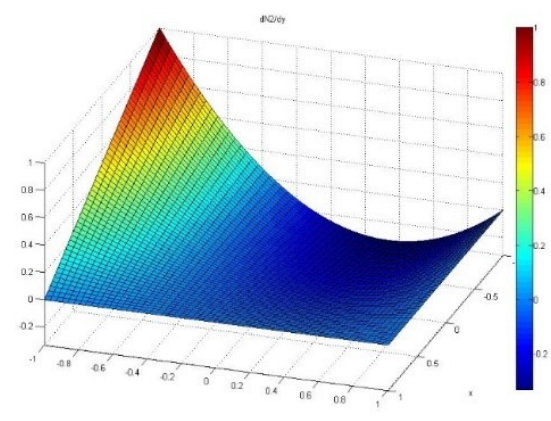

(c)

Figure 14 Graph of DFE shape function derivative $\frac{\partial N_{3, \omega}}{\partial y}$ for value of $\gamma_{x y}=10 e-6$ (a) and for value of $\gamma_{x y}=2$ (b) and third Hermite shape function derivative with respect to $x$ (c) over the plate element of Figure 3 


$$
\begin{aligned}
& N_{4, \omega}=\frac{\sin \left(\gamma_{x y} x\right)-\sinh \left(\gamma_{x y} x\right)}{4\left(\sin \left(\gamma_{x y}\right)-\sinh \left(\gamma_{x y}\right)-\gamma_{x y} \cos \left(\gamma_{x y}\right)+\gamma_{x y} \cosh \left(\gamma_{x y}\right)\right)}-\frac{\sin \left(\gamma_{x y} y\right)-\sinh \left(\gamma_{x y} y\right)}{4\left(\sin \left(\gamma_{x y}\right)-\sinh \left(\gamma_{x y}\right)-\gamma_{x y} \cos \left(\gamma_{x y}\right)+\gamma_{x y} \cosh \left(\gamma_{x y}\right)\right)} \\
& -\frac{\sin \left(\gamma_{x y}\right)\left(\cos \left(\gamma_{x y} x\right)-\cosh \left(\gamma_{x y} x\right)\right)}{8\left(\cos \left(\gamma_{x y}\right) \sinh \left(\gamma_{x y}\right)+\cosh \left(\gamma_{x y}\right) \sin \left(\gamma_{x y}\right)\right)}-\frac{\sin \left(\gamma_{x y}\right)\left(\cos \left(\gamma_{x y} y\right)-\cosh \left(\gamma_{x y} y\right)\right)}{8\left(\cos \left(\gamma_{x y}\right) \sinh \left(\gamma_{x y}\right)+\cosh \left(\gamma_{x y}\right) \sin \left(\gamma_{x y}\right)\right)} \\
& +\frac{\left(\sin \left(\gamma_{x y}\right)+\sinh \left(\gamma_{x y}\right)\right)\left(\cos \left(\gamma_{x y} x\right)+\cos \left(\gamma_{x y} y\right)\right)}{8\left(\cos \left(\gamma_{x y}\right) \sinh \left(\gamma_{x y}\right)+\cosh \left(\gamma_{x y}\right) \sin \left(\gamma_{x y}\right)\right)} \\
& -\frac{\left(x \sin \left(\gamma_{x y} y\right)+y \sin \left(\gamma_{x y} x\right)\right)\left(\sin \left(\gamma_{x y}\right)-\sinh \left(\gamma_{x y}\right)+\gamma_{x y} \cos \left(\gamma_{x y}\right)-\gamma_{x y} \cosh \left(\gamma_{x y}\right)\right)}{8 \gamma_{x y}\left(\cos \left(\gamma_{x y}\right) \sinh \left(\gamma_{x y}\right)-\cosh \left(\gamma_{x y}\right) \sin \left(\gamma_{x y}\right)\right)} \\
& +\frac{y\left(\sin \left(\gamma_{x y} x\right)-\sinh \left(\gamma_{x y} x\right)\right)\left(\sin \left(\gamma_{x y}\right)+\gamma_{x y} \cos \left(\gamma_{x y}\right)\right)}{8 \gamma_{x y}\left(\cos \left(\gamma_{x y}\right) \sinh \left(\gamma_{x y}\right)-\cosh \left(\gamma_{x y}\right) \sin \left(\gamma_{x y}\right)\right)}+\frac{\left.\left.8 \gamma_{x y} y\right)-\sinh \left(\gamma_{x y} y\right)\right)\left(\sin \left(\gamma_{x y}\right)+\gamma_{x y} \cos \left(\gamma_{x y}\right)\right)}{8\left(\cos \left(\gamma_{x y}\right) \sinh \left(\gamma_{x y}\right)+\cosh \left(\gamma_{x y}\right) \sin \left(\gamma_{x y}\right)\right)\left(\sin \left(\gamma_{x y}\right)-\sinh \left(\gamma_{x y}\right)-\gamma_{x y} \cos \left(\gamma_{x y}\right)+\gamma_{x y} \cosh \left(\gamma_{x y}\right)\right)} \\
& -\frac{\left.\gamma_{x y} y\left(\gamma_{x y}\right)\right)}{4\left(\cos \left(\gamma_{x y}\right) \sinh \left(\gamma_{x y}\right)+\cosh \left(\gamma_{x y}\right) \sin \left(\gamma_{x y}\right)\right)\left(\sin \left(\gamma_{x y}\right)-\sinh \left(\gamma_{x y}\right)-\gamma_{x y} \cos \left(\gamma_{x y}\right)+\gamma_{x y} \cosh \left(\gamma_{x y}\right)\right)} \\
& +\frac{\gamma_{x y} y \cos \left(\gamma_{x y} x\right)\left(\sin \left(\gamma_{x y}\right)+\sinh \left(\gamma_{x y}\right)\right)\left(\cos \left(\gamma_{x y}\right)-\cosh \left(\gamma_{x y}\right)\right)}{4\left(\cos \left(\gamma_{x y}\right) \sinh \left(\gamma_{x y}\right)+\cosh \left(\gamma_{x y}\right) \sin \left(\gamma_{x y}\right)\right)\left(\sin \left(\gamma_{x y}\right)-\sinh \left(\gamma_{x y}\right)-\gamma_{x y} \cos \left(\gamma_{x y}\right)+\gamma_{x y} \cosh \left(\gamma_{x y}\right)\right)} \\
& +\frac{\gamma_{x y} x \sin \left(\gamma_{x y}\right)\left(\cos \left(\gamma_{x y} y\right)-\cosh \left(\gamma_{x y} y\right)\right)\left(\cos \left(\gamma_{x y}\right)-\cosh \left(\gamma_{x y}\right)\right)}{4\left(\cos \left(\gamma_{x y}\right) \sinh \left(\gamma_{x y}\right)+\cosh \left(\gamma_{x y}\right) \sin \left(\gamma_{x y}\right)\right)\left(\sin \left(\gamma_{x y}\right)-\sinh \left(\gamma_{x y}\right)-\gamma_{x y} \cos \left(\gamma_{x y}\right)+\gamma_{x y} \cosh \left(\gamma_{x y}\right)\right)}
\end{aligned}
$$




$$
N_{4}=\frac{(x+1)(y-1)\left(x^{2}-x+y^{2}+y-2\right)}{8}
$$

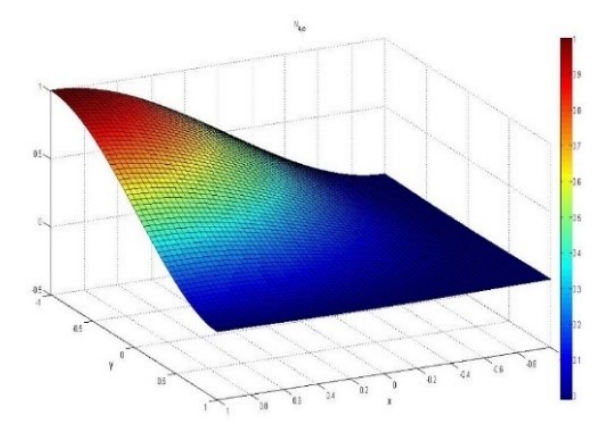

(a)

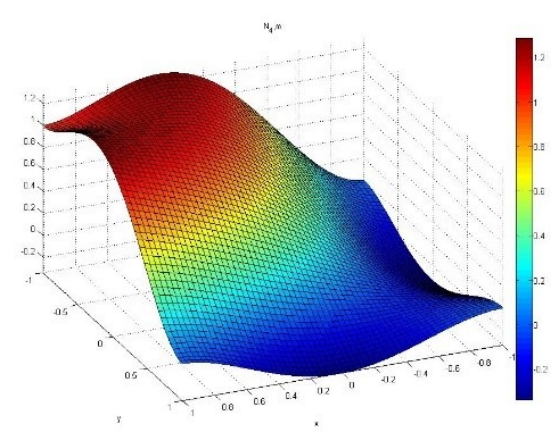

(b)

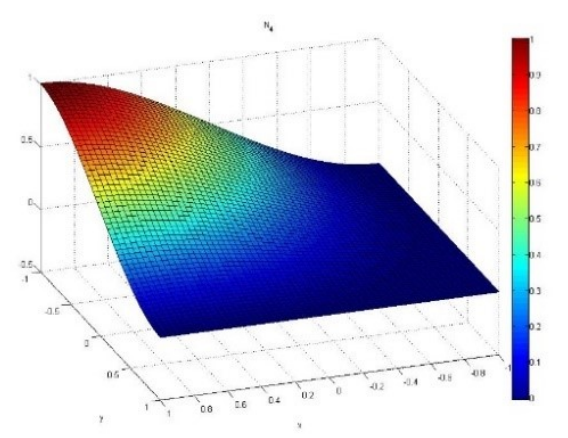

(c)

Figure 15 Graph of DFE shape function $N_{4, \omega}$ for value of $\gamma_{x y}=10 e-6$ (a) and for value of $\gamma_{x y}=2$ (b) and fourth Hermite shape function (c) over the plate element of Figure 3 


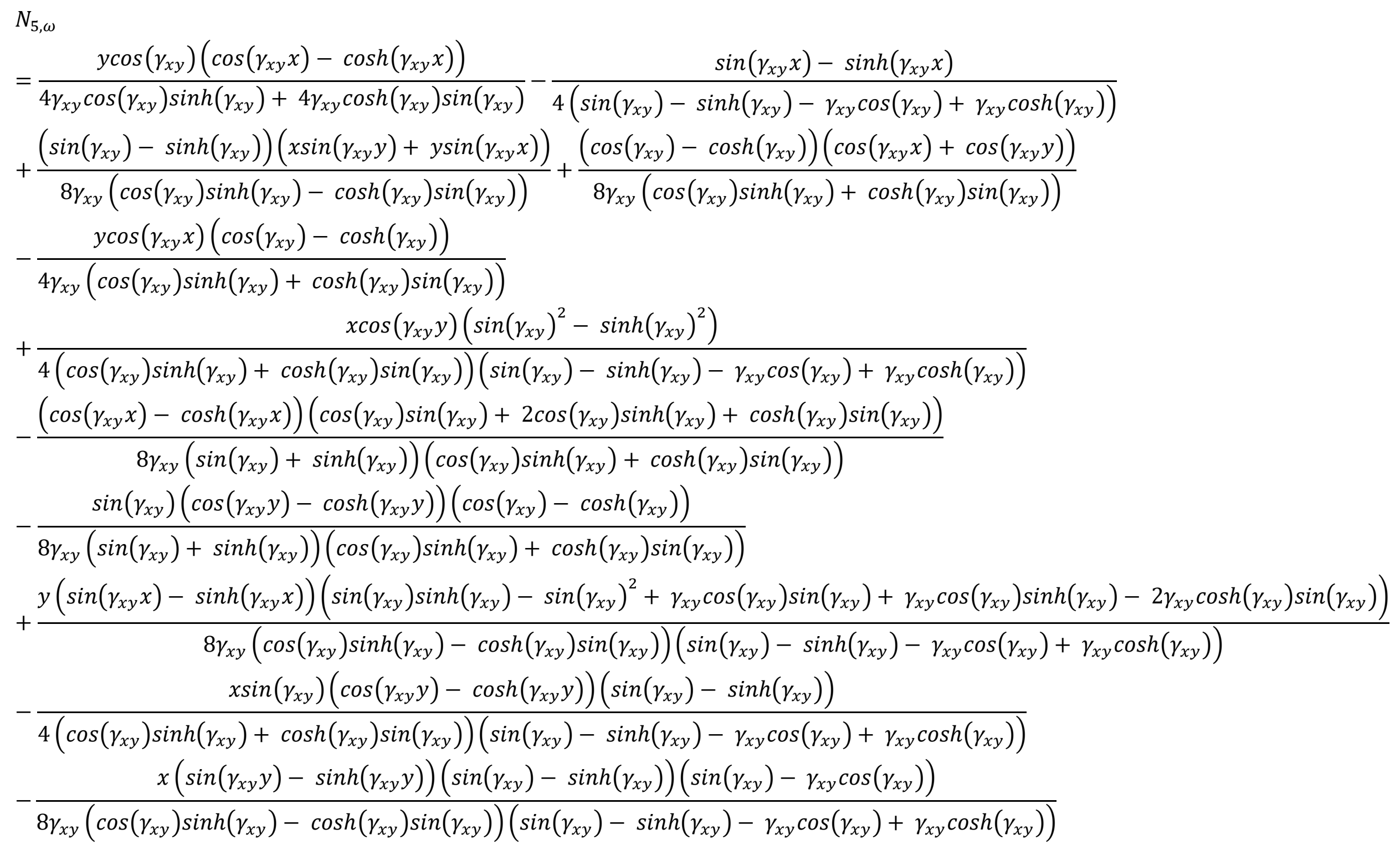




$$
N_{5}=-\frac{(x-1)(x+1)^{2}(y-1)}{8}
$$

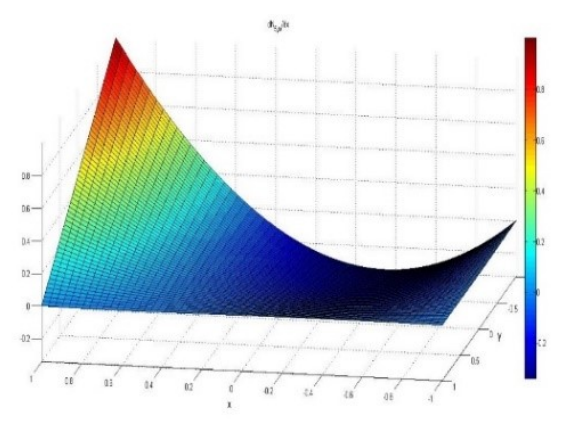

(a)

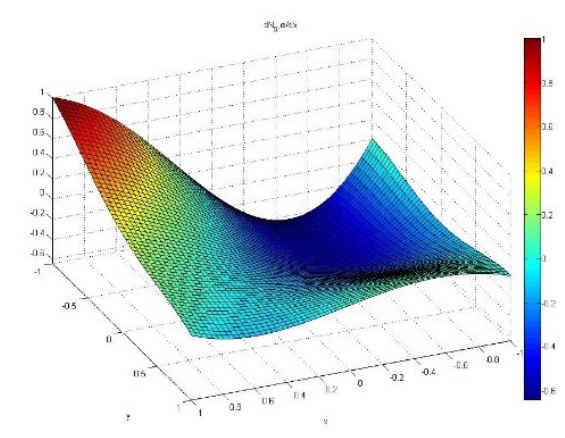

(b)

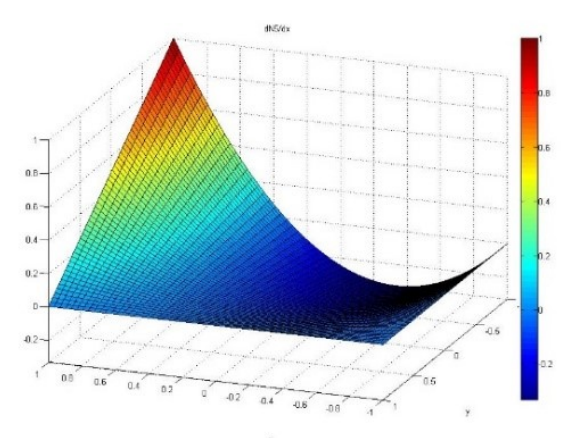

(c)

Figure 16 Graph of DFE shape function derivative $\frac{\partial N_{5, \omega}}{\partial x}$ for value of $\gamma_{x y}=10 e-6$ (a) and for value of $\gamma_{x y}=2$ (b) and fifth Hermite shape function derivative with respect to $x$ (c) over the plate element of Figure 3 


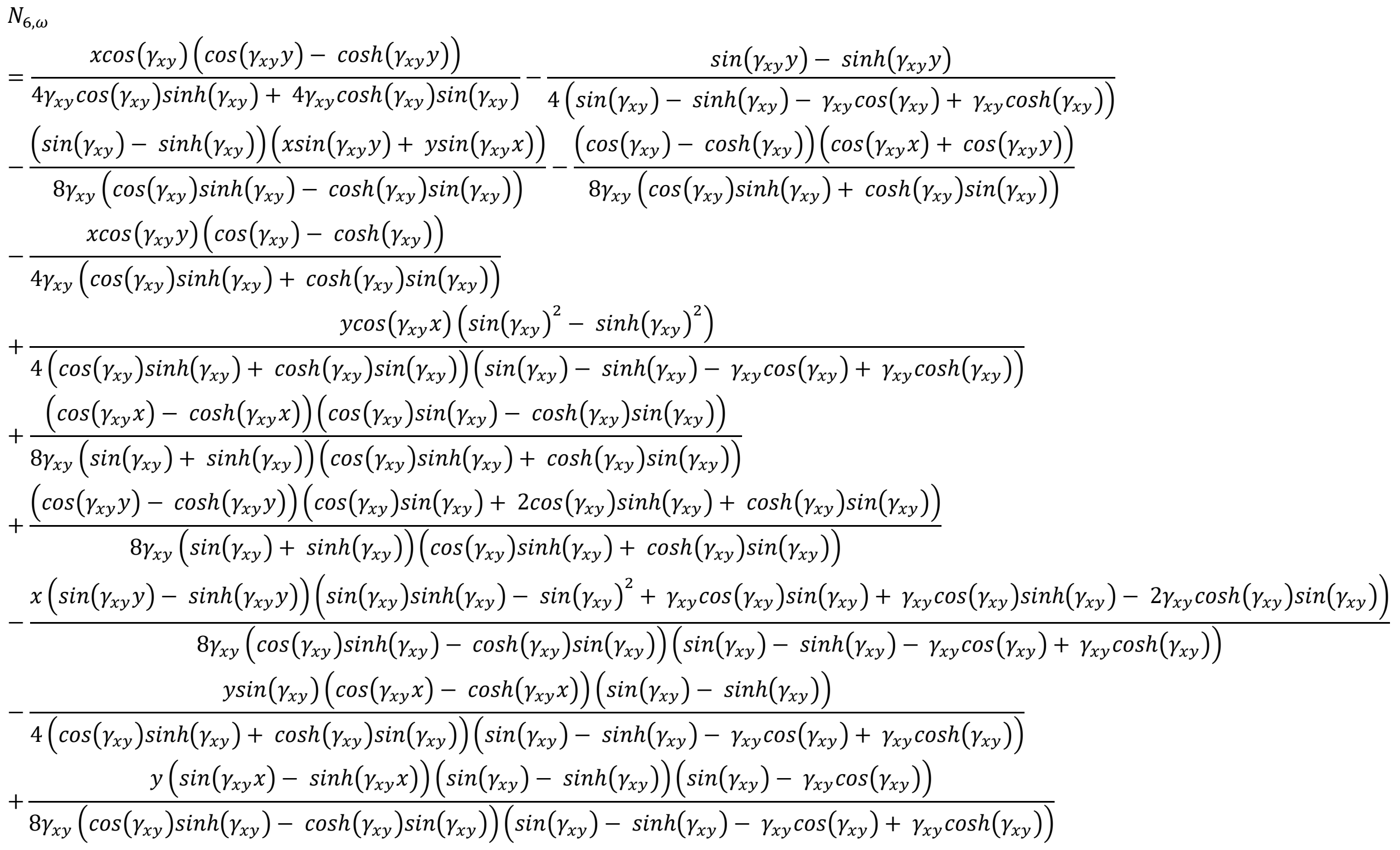




$$
N_{6}=\frac{(x+1)(y-1)^{2}(y+1)}{8}
$$

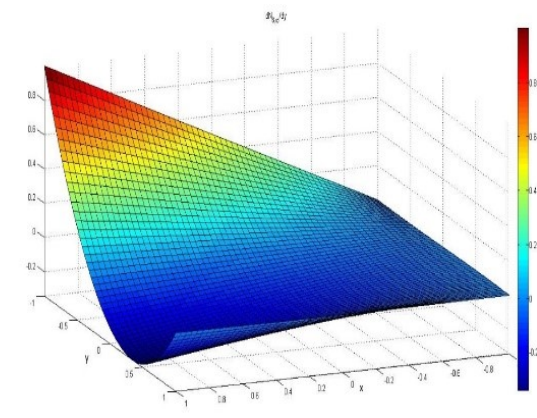

(a)

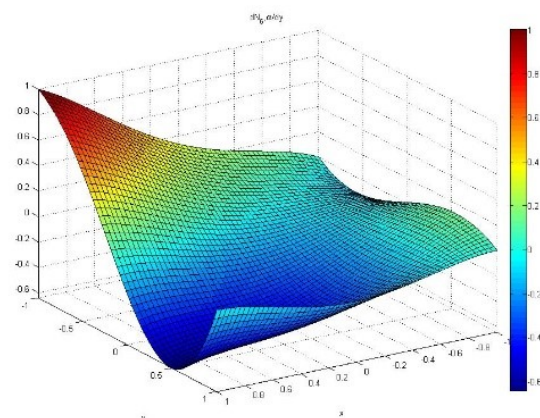

(b)

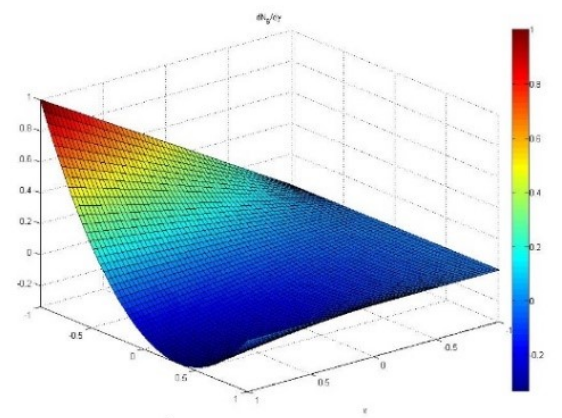

(c)

Figure 17 Graph of DFE shape function derivative $\frac{\partial N_{6, \omega}}{\partial y}$ for value of $\gamma_{x y}=10 e-6$ (a) and for value of $\gamma_{x y}=2$ (b) and sixth Hermite shape function derivative with respect to $y$ (c) over the plate element of Figure 3 


$$
\begin{aligned}
& N_{7, \omega}=\frac{\sin \left(\gamma_{x y} x\right)-\sinh \left(\gamma_{x y} x\right)}{4\left(\sin \left(\gamma_{x y}\right)-\sinh \left(\gamma_{x y}\right)-\gamma_{x y} \cos \left(\gamma_{x y}\right)+\gamma_{x y} \cosh \left(\gamma_{x y}\right)\right)}+\frac{\sin \left(\gamma_{x y} y\right)-\sinh \left(\gamma_{x y} y\right)}{4\left(\sin \left(\gamma_{x y}\right)-\sinh \left(\gamma_{x y}\right)-\gamma_{x y} \cos \left(\gamma_{x y}\right)+\gamma_{x y} \cosh \left(\gamma_{x y}\right)\right)} \\
& -\frac{\sin \left(\gamma_{x y}\right)\left(\cos \left(\gamma_{x y} x\right)-\cosh \left(\gamma_{x y} x\right)\right)}{8\left(\cos \left(\gamma_{x y}\right) \sinh \left(\gamma_{x y}\right)+\cosh \left(\gamma_{x y}\right) \sin \left(\gamma_{x y}\right)\right)}-\frac{\sin \left(\gamma_{x y}\right)\left(\cos \left(\gamma_{x y} y\right)-\cosh \left(\gamma_{x y} y\right)\right)}{8\left(\cos \left(\gamma_{x y}\right) \sinh \left(\gamma_{x y}\right)+\cosh \left(\gamma_{x y}\right) \sin \left(\gamma_{x y}\right)\right)} \\
& +\frac{\left(\sin \left(\gamma_{x y}\right)+\sinh \left(\gamma_{x y}\right)\right)\left(\cos \left(\gamma_{x y} x\right)+\cos \left(\gamma_{x y} y\right)\right)}{8\left(\cos \left(\gamma_{x y}\right) \sinh \left(\gamma_{x y}\right)+\cosh \left(\gamma_{x y}\right) \sin \left(\gamma_{x y}\right)\right)} \\
& +\frac{\left(x \sin \left(\gamma_{x y} y\right)+y \sin \left(\gamma_{x y} x\right)\right)\left(\sin \left(\gamma_{x y}\right)-\sinh \left(\gamma_{x y}\right)+\gamma_{x y} \cos \left(\gamma_{x y}\right)-\gamma_{x y} \cosh \left(\gamma_{x y}\right)\right)}{8 \gamma_{x y}\left(\cos \left(\gamma_{x y}\right) \sinh \left(\gamma_{x y}\right)-\cosh \left(\gamma_{x y}\right) \sin \left(\gamma_{x y}\right)\right)} \\
& -\frac{y\left(\sin \left(\gamma_{x y} x\right)-\sinh \left(\gamma_{x y} x\right)\right)\left(\sin \left(\gamma_{x y}\right)+\gamma_{x y} \cos \left(\gamma_{x y}\right)\right)}{8 \gamma_{x y}\left(\cos \left(\gamma_{x y}\right) \sinh \left(\gamma_{x y}\right)-\cosh \left(\gamma_{x y}\right) \sin \left(\gamma_{x y}\right)\right)}-\frac{\left.\left.x \gamma_{x y} y\right)-\sinh \left(\gamma_{x y} y\right)\right)\left(\sin \left(\gamma_{x y}\right)+\gamma_{x y} \cos \left(\gamma_{x y}\right) \sinh \left(\gamma_{x y}\right)-\cosh \left(\gamma_{x y}\right) \sin \left(\gamma_{x y}\right)\right)}{8\left(\cos \left(\gamma_{x y}\right) \sinh \left(\gamma_{x y}\right)+\cosh \left(\gamma_{x y}\right) \sin \left(\gamma_{x y}\right)\right)\left(\sin \left(\gamma_{x y}\right)-\sinh \left(\gamma_{x y}\right)-\gamma_{x y} \cos \left(\gamma_{x y}\right)+\gamma_{x y} \cosh \left(\gamma_{x y}\right)\right)} \\
& -\frac{\gamma_{x y} x \cos \left(\gamma_{x y} y\right)\left(\sin \left(\gamma_{x y}\right)+\sinh \left(\gamma_{x y}\right)\right)\left(\cos \left(\gamma_{x y}\right)-\cosh \left(\gamma_{x y}\right)\right)}{4\left(\cos \left(\gamma_{x y}\right) \sinh \left(\gamma_{x y}\right)+\cosh \left(\gamma_{x y}\right) \sin \left(\gamma_{x y}\right)\right)\left(\sin \left(\gamma_{x y}\right)-\sinh \left(\gamma_{x y}\right)-\gamma_{x y} \cos \left(\gamma_{x y}\right)+\gamma_{x y} \cosh \left(\gamma_{x y}\right)\right)} \\
& +\frac{\gamma_{x y} y \cos \left(\gamma_{x y} x\right)\left(\sin \left(\gamma_{x y}\right)+\sinh \left(\gamma_{x y}\right)\right)\left(\cos \left(\gamma_{x y}\right)-\cosh \left(\gamma_{x y}\right)\right)}{4\left(\cos \left(\gamma_{x y}\right) \sinh \left(\gamma_{x y}\right)+\cosh \left(\gamma_{x y}\right) \sin \left(\gamma_{x y}\right)\right)\left(\sin \left(\gamma_{x y}\right)-\sinh \left(\gamma_{x y}\right)-\gamma_{x y} \cos \left(\gamma_{x y}\right)+\gamma_{x y} \cosh \left(\gamma_{x y}\right)\right)} \\
& +\frac{\gamma_{x y} x \sin \left(\gamma_{x y}\right)\left(\cos \left(\gamma_{x y} y\right)-\cosh \left(\gamma_{x y} y\right)\right)\left(\cos \left(\gamma_{x y}\right)-\cosh \left(\gamma_{x y}\right)\right)}{4\left(\cos \left(\gamma_{x y}\right) \sinh \left(\gamma_{x y}\right)+\cosh \left(\gamma_{x y}\right) \sin \left(\gamma_{x y}\right)\right)\left(\sin \left(\gamma_{x y}\right)-\sinh \left(\gamma_{x y}\right)-\gamma_{x y} \cos \left(\gamma_{x y}\right)+\gamma_{x y} \cosh \left(\gamma_{x y}\right)\right)}
\end{aligned}
$$




$$
\text { 니빈 }
$$




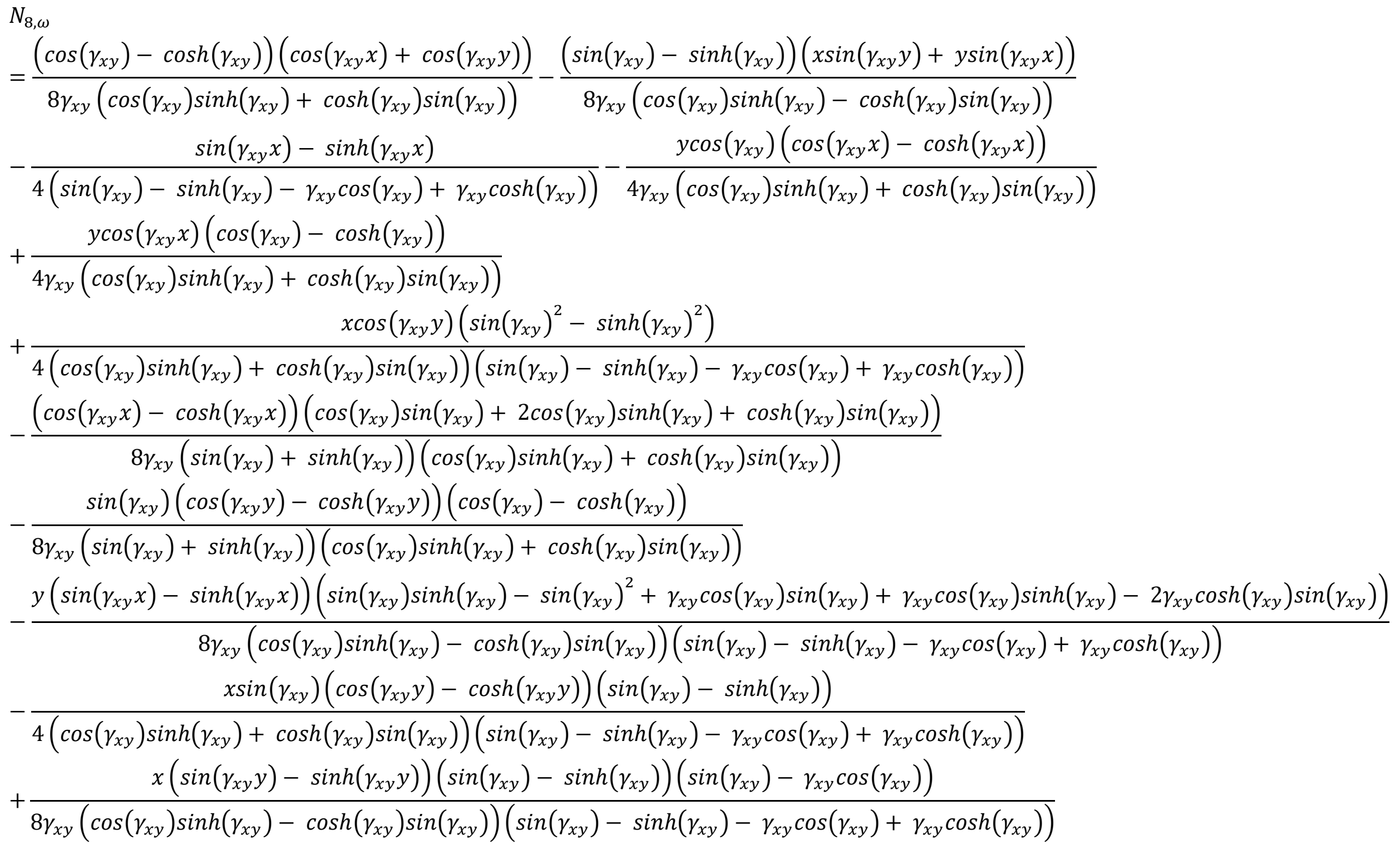


$N_{8}=\frac{(x-1)(x+1)^{2}(y+1)}{8}$

A- 16

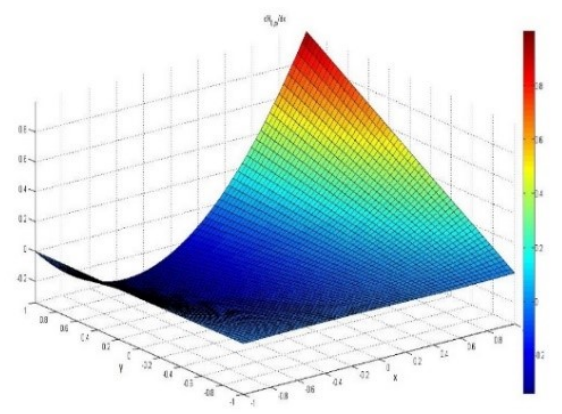

(a)

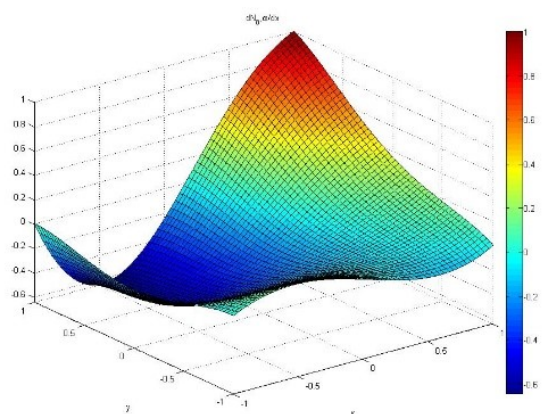

(b)

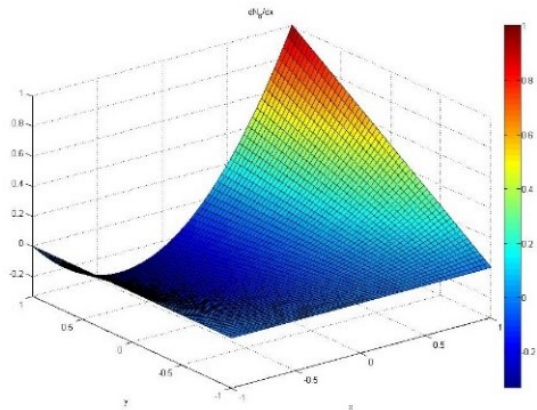

(c)

Figure 19 Graph of DFE shape function derivative $\frac{\partial N_{8, \omega}}{\partial x}$ for value of $\gamma_{x y}=10 e-6$ (a) and for value of $\gamma_{x y}=2$ (b) and eighth Hermite shape function derivative with respect to $x$ (c) over the plate element of Figure 3 


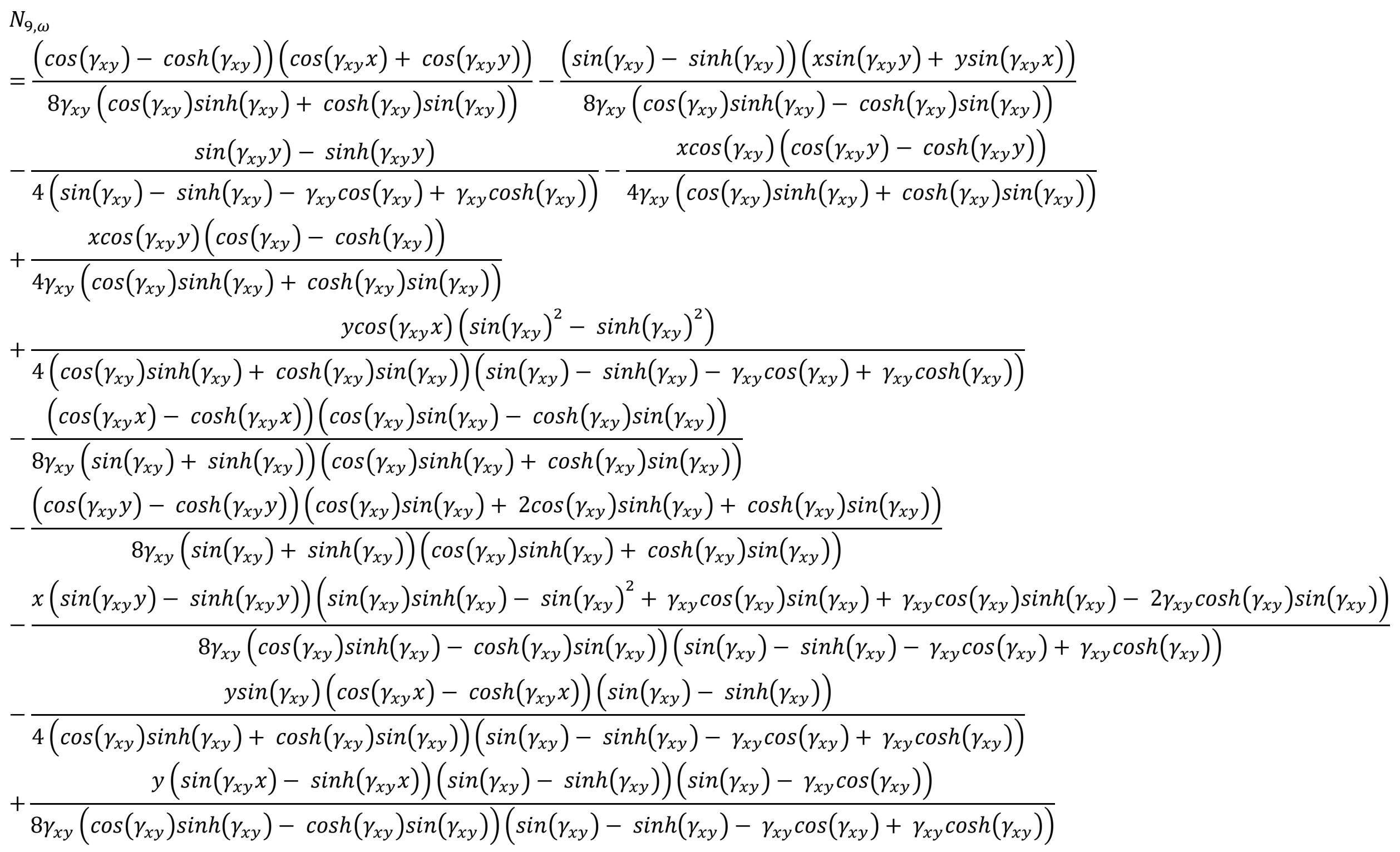




$$
N_{9}=\frac{(x+1)(y-1)(y+1)^{2}}{8}
$$

A- 18

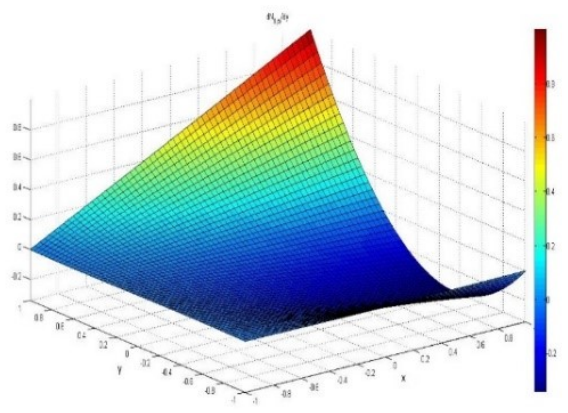

(a)

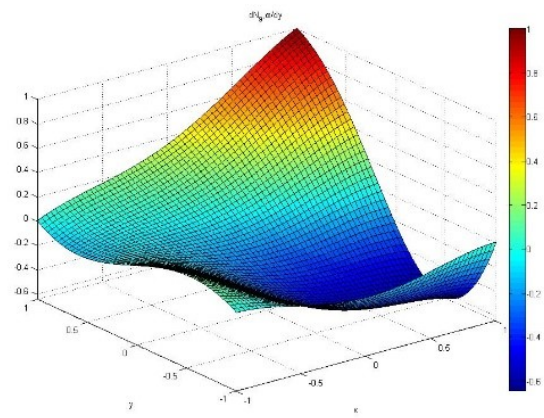

(b)

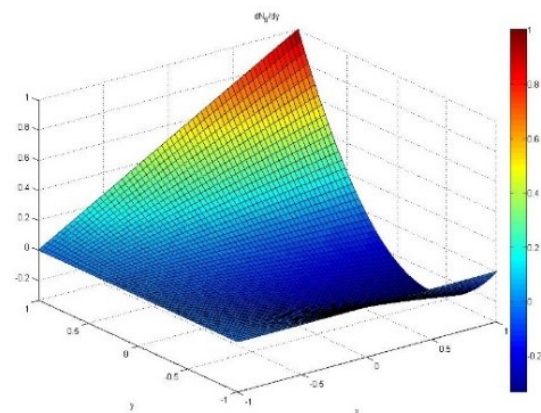

(c)

Figure 20 Graph of DFE shape function derivative $\frac{\partial N_{9, \omega}}{\partial y}$ for value of $\gamma_{x y}=10 e-6$ (a) and for value of $\gamma_{x y}=2$ (b) and ninth Hermite shape function derivative with respect to $y$ (c) over the plate element of Figure 3 


$$
\begin{aligned}
& N_{10, \omega}=\frac{\sin \left(\gamma_{x y} y\right)-\sinh \left(\gamma_{x y} y\right)}{4\left(\sin \left(\gamma_{x y}\right)-\sinh \left(\gamma_{x y}\right)-\gamma_{x y} \cos \left(\gamma_{x y}\right)+\gamma_{x y} \cosh \left(\gamma_{x y}\right)\right)}-\frac{\sin \left(\gamma_{x y} x\right)-\sinh \left(\gamma_{x y} x\right)}{4\left(\sin \left(\gamma_{x y}\right)-\sinh \left(\gamma_{x y}\right)-\gamma_{x y} \cos \left(\gamma_{x y}\right)+\gamma_{x y} \cosh \left(\gamma_{x y}\right)\right)} \\
& -\frac{\sin \left(\gamma_{x y}\right)\left(\cos \left(\gamma_{x y} x\right)-\cosh \left(\gamma_{x y} x\right)\right)}{8\left(\cos \left(\gamma_{x y}\right) \sinh \left(\gamma_{x y}\right)+\cosh \left(\gamma_{x y}\right) \sin \left(\gamma_{x y}\right)\right)}-\frac{\sin \left(\gamma_{x y}\right)\left(\cos \left(\gamma_{x y} y\right)-\cosh \left(\gamma_{x y} y\right)\right)}{8\left(\cos \left(\gamma_{x y}\right) \sinh \left(\gamma_{x y}\right)+\cosh \left(\gamma_{x y}\right) \sin \left(\gamma_{x y}\right)\right)} \\
& +\frac{\left(\sin \left(\gamma_{x y}\right)+\sinh \left(\gamma_{x y}\right)\right) *\left(\cos \left(\gamma_{x y} x\right)+\cos \left(\gamma_{x y} y\right)\right)}{8\left(\cos \left(\gamma_{x y}\right) \sinh \left(\gamma_{x y}\right)+\cosh \left(\gamma_{x y}\right) \sin \left(\gamma_{x y}\right)\right)} \\
& -\frac{\left(x \sin \left(\gamma_{x y} y\right)+y \sin \left(\gamma_{x y} x\right)\right)\left(\sin \left(\gamma_{x y}\right)-\sinh \left(\gamma_{x y}\right)+\gamma_{x y} \cos \left(\gamma_{x y}\right)-\gamma_{x y} \cosh \left(\gamma_{x y}\right)\right)}{8 \gamma_{x y}\left(\cos \left(\gamma_{x y}\right) \sinh \left(\gamma_{x y}\right)-\cosh \left(\gamma_{x y}\right) \sin \left(\gamma_{x y}\right)\right)} \\
& +\frac{y\left(\sin \left(\gamma_{x y} x\right)-\sinh \left(\gamma_{x y} x\right)\right)\left(\sin \left(\gamma_{x y}\right)+\gamma_{x y} \cos \left(\gamma_{x y}\right)\right)}{8 \gamma_{x y}\left(\cos \left(\gamma_{x y}\right) \sinh \left(\gamma_{x y}\right)-\cosh \left(\gamma_{x y}\right) \sin \left(\gamma_{x y}\right)\right)}+\frac{\left.\left.x \gamma_{x y} y\right)-\sinh \left(\gamma_{x y} y\right)\right)\left(\sin \left(\gamma_{x y}\right)+\gamma_{x y} \cos \left(\gamma_{x y}\right) \sinh \left(\gamma_{x y}\right)-\cosh \left(\gamma_{x y}\right) \sin \left(\gamma_{x y}\right)\right)}{8\left(\cos \left(\gamma_{x y}\right) \sinh \left(\gamma_{x y}\right)+\cosh \left(\gamma_{x y}\right) \sin \left(\gamma_{x y}\right)\right)\left(\sin \left(\gamma_{x y}\right)-\sinh \left(\gamma_{x y}\right)-\gamma_{x y} \cos \left(\gamma_{x y}\right)+\gamma_{x y} \cosh \left(\gamma_{x y}\right)\right)} \\
& +\frac{\gamma_{x y} x \cos \left(\gamma_{x y} y\right)\left(\sin \left(\gamma_{x y}\right)+\sinh \left(\gamma_{x y}\right)\right)\left(\cos \left(\gamma_{x y}\right)-\cosh \left(\gamma_{x y}\right)\right)}{4\left(\cos \left(\gamma_{x y}\right) \sinh \left(\gamma_{x y}\right)+\cosh \left(\gamma_{x y}\right) * \sin \left(\gamma_{x y}\right)\right)\left(\sin \left(\gamma_{x y}\right)-\sinh \left(\gamma_{x y}\right)-\gamma_{x y} \cos \left(\gamma_{x y}\right)+\gamma_{x y} \cosh \left(\gamma_{x y}\right)\right)} \\
& -\frac{\gamma_{x y} y \cos \left(\gamma_{x y} x\right)\left(\sin \left(\gamma_{x y}\right)+\sinh \left(\gamma_{x y}\right)\right)\left(\cos \left(\gamma_{x y}\right)-\cosh \left(\gamma_{x y}\right)\right)}{4\left(\cos \left(\gamma_{x y}\right) \sinh \left(\gamma_{x y}\right)+\cosh \left(\gamma_{x y}\right) \sin \left(\gamma_{x y}\right)\right)\left(\sin \left(\gamma_{x y}\right)-\sinh \left(\gamma_{x y}\right)-\gamma_{x y} \cos \left(\gamma_{x y}\right)+\gamma_{x y} \cosh \left(\gamma_{x y}\right)\right)} \\
& -\frac{\gamma_{x y} x \sin \left(\gamma_{x y}\right)\left(\cos \left(\gamma_{x y} y\right)-\cosh \left(\gamma_{x y} y\right)\right)\left(\cos \left(\gamma_{x y}\right)-\cosh \left(\gamma_{x y}\right)\right)}{4\left(\cos \left(\gamma_{x y}\right) \sinh \left(\gamma_{x y}\right)+\cosh \left(\gamma_{x y}\right) \sin \left(\gamma_{x y}\right)\right)\left(\sin \left(\gamma_{x y}\right)-\sinh \left(\gamma_{x y}\right)-\gamma_{x y} \cos \left(\gamma_{x y}\right)+\gamma_{x y} \cosh \left(\gamma_{x y}\right)\right)}
\end{aligned}
$$




$$
N_{10}=\frac{(x+1)(y-1)(y+1)^{2}}{8}
$$

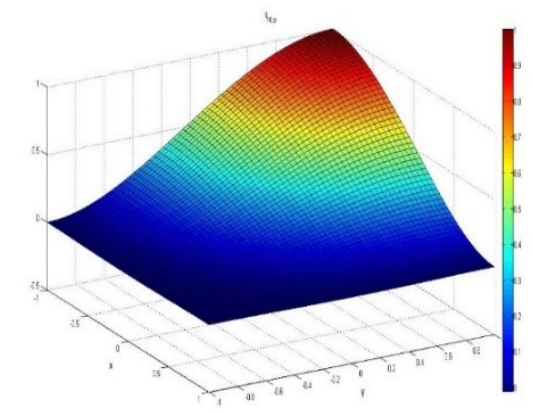

(a)

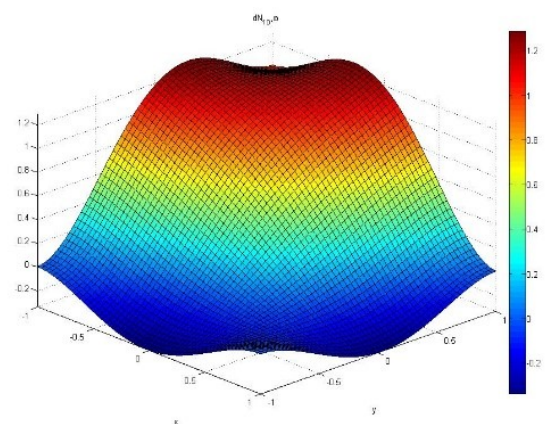

(b)

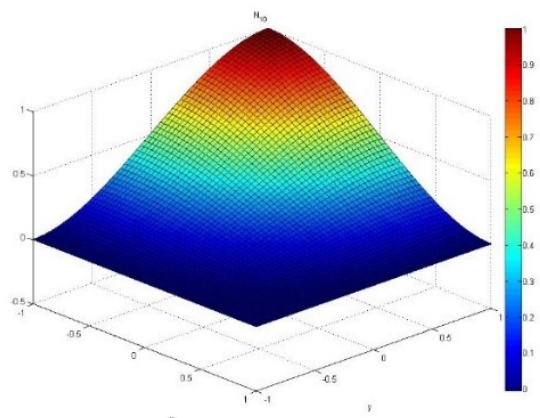

(c)

Figure 21 Graph of DFE shape function $N_{10, \omega}$ for value of $\gamma_{x y}=10 e-6$ (a) and for value of $\gamma_{x y}=2$ (b) and tenth Hermite shape function (c) over the plate element of Figure 3 


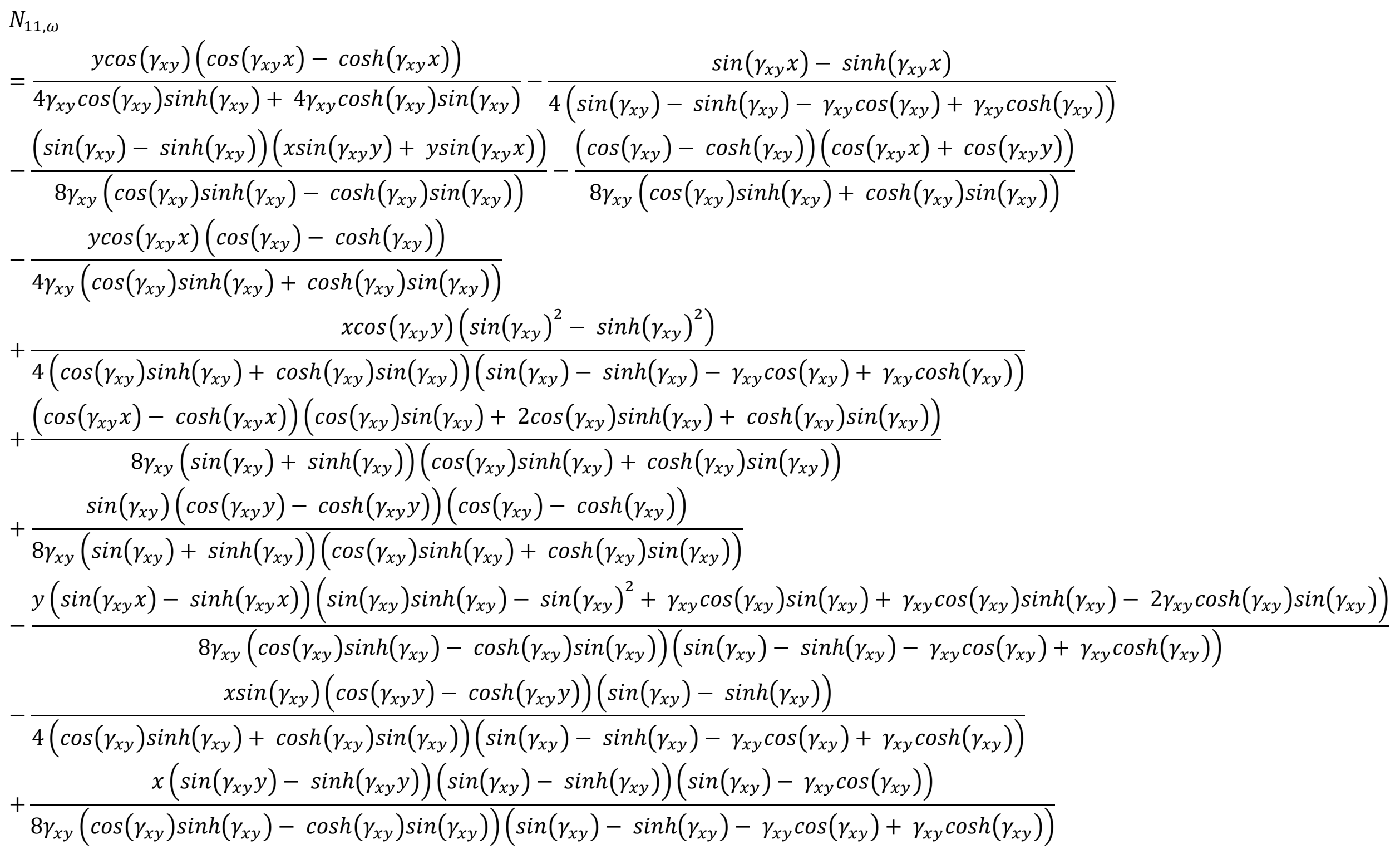




$$
N_{11}=\frac{(x-1)^{2}(x+1)(y+1)}{8}
$$

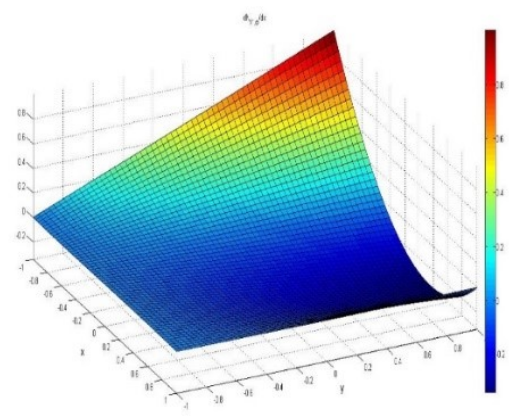

(a)

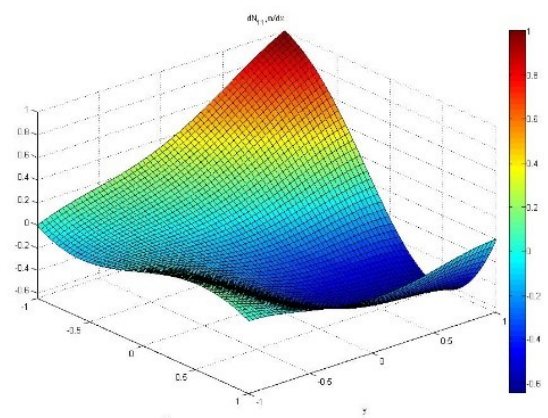

(b)

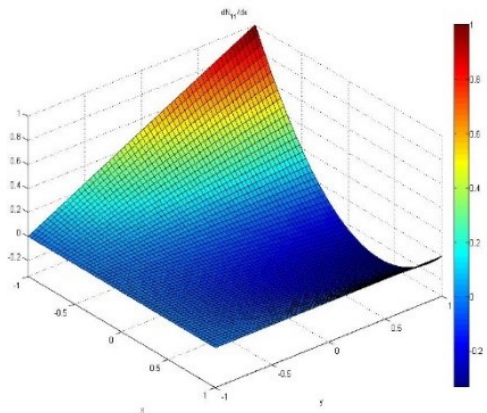

(c)

Figure 22 Graph of DFE shape function derivative $\frac{\partial N_{11, \omega}}{\partial x}$ for value of $\gamma_{x y}=10 e-6$ (a) and for value of $\gamma_{x y}=2$ (b) and eleventh Hermite shape function derivative with respect to $x$ (c) over the plate element of Figure 3 


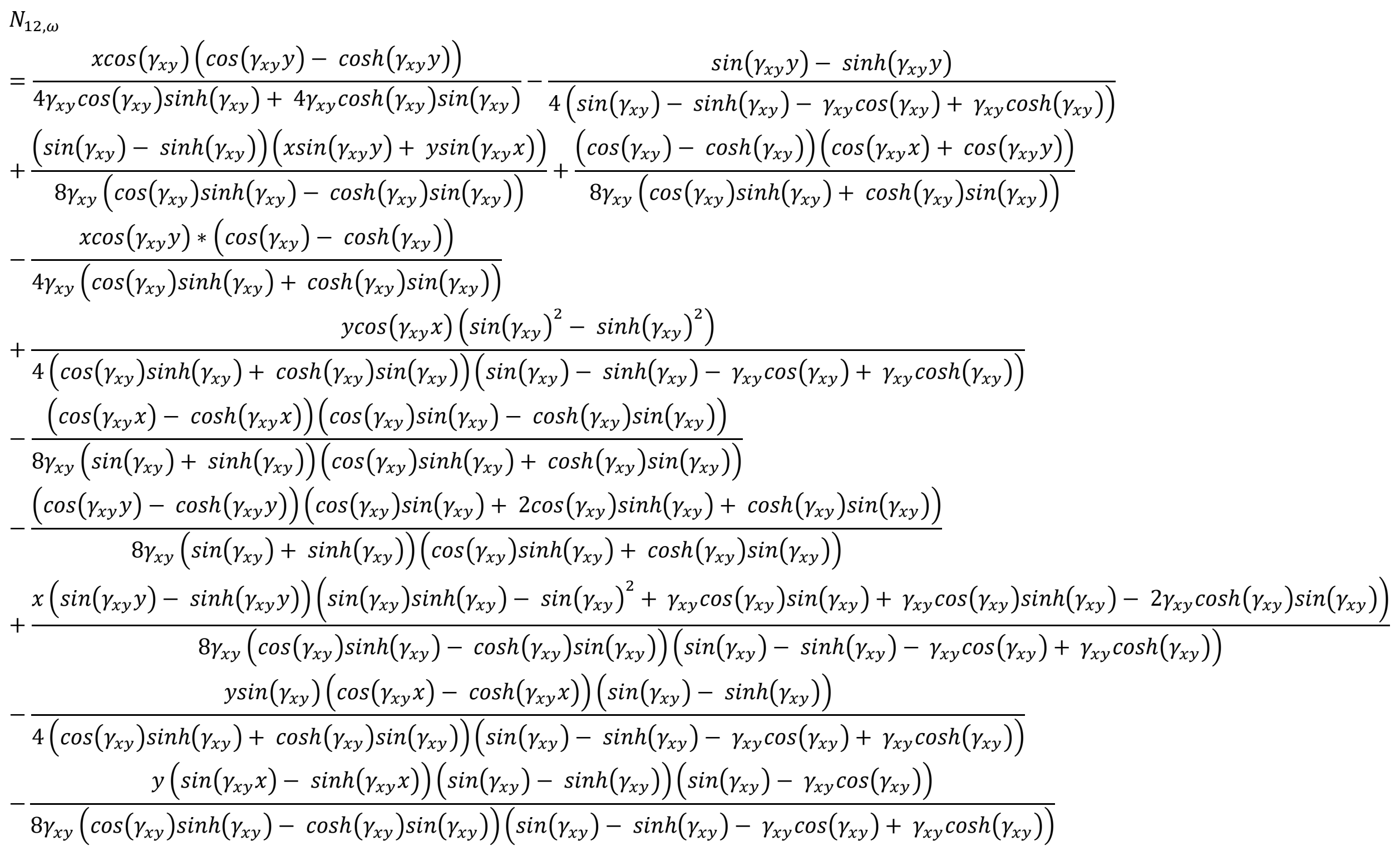




$$
N_{12}=-\frac{(x-1)(y-1)(y+1)^{2}}{8}
$$

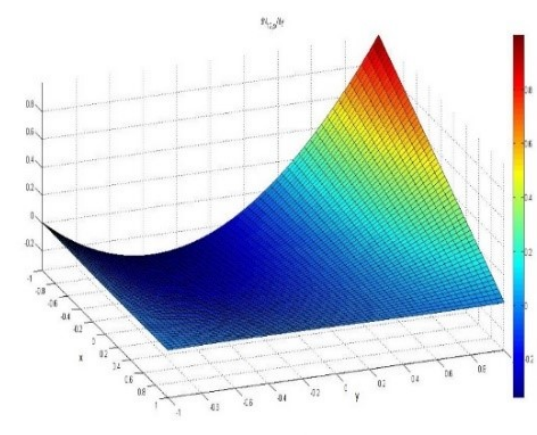

(a)

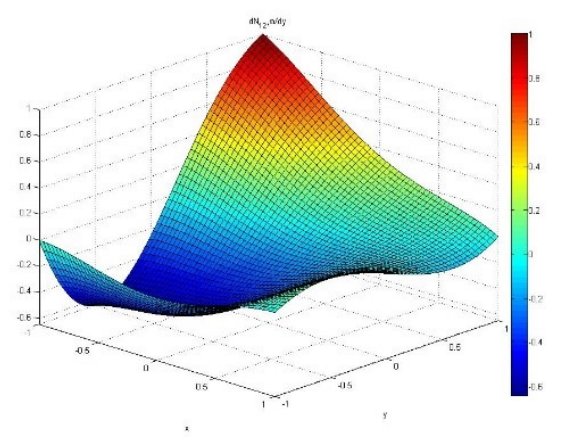

(b)

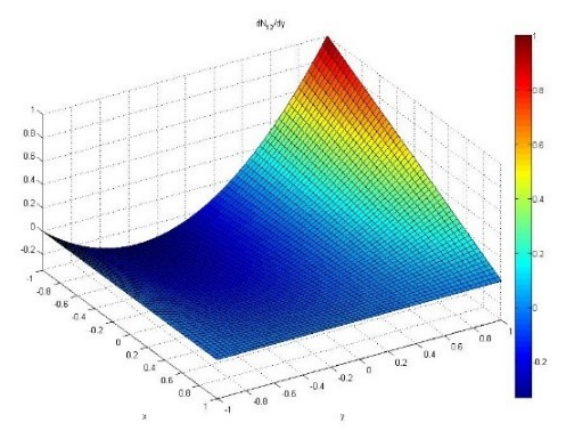

(c)

Figure 23 Graph of DFE shape function derivative $\frac{\partial N_{12, \omega}}{\partial y}$ for value of $\gamma_{x y}=10 e-6$ (a) and for value of $\gamma_{x y}=2$ (b) and eleventh Hermite shape function derivative with respect to $y$ (c) over the plate element of Figure 3 


\section{APPENDIX B}

In this appendix, the DFE matrix developed is extended to plates of arbitrary shapes. The absence of area integrals in final form of the DFE equation is particularly important in this extension, as the cumbersome and inaccurate conversion of area integrals through Jacobian matrices is not required. The derivation in this appendix are not implemented over an element, and serve as a guideline for future DFE development with more enriched shape functions.

To perform the extension, quadrilateral case shown in Figure $24 \mathrm{~b}$ is considered as development framework. To arrive at a general method that can be applied to any quadrilateral, a mapping is used to convert the element variables to a reference square, called the natural coordinate system. By doing so, the final element evaluation will be performed in a universal reference frame, shown in Figure 24a regardless of element's specific shape, and therefore can be used to develop a general algorithm for various geometries. Similar procedure can be followed for any geometry, as the quadrilateral relations do not influence the derivation process, and are used as an example.

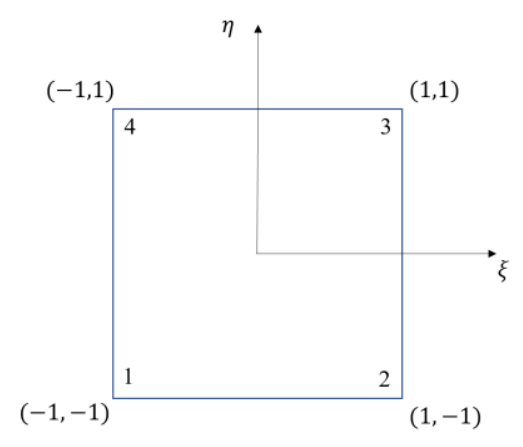

(a)

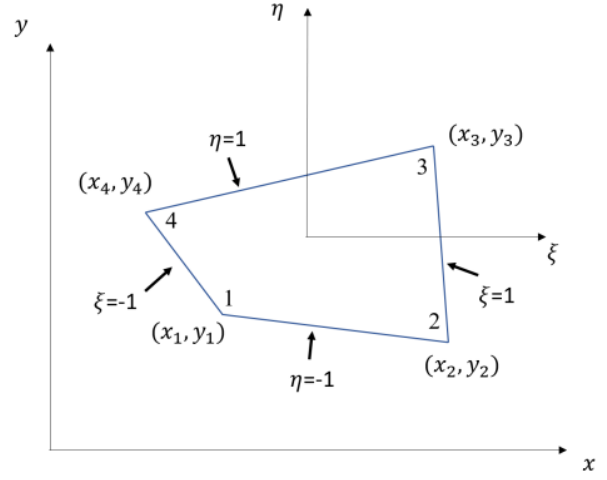

(b)

Figure 24 Natural coordinate system (a) is used to map arbitrary shapes from element coordinate system (b) on a 2 by 2 square.

For the quadrilateral shown above, the variables $x$ and $y$ from element coordinate system, can be related to the variables $\xi$ and $\eta$ from natural coordinate system, using bi-linear relations:

$x=a_{0}+a_{1} \xi+a_{2} \eta+a_{3} \xi \eta$

B- 1

$y=b_{0}+b_{1} \xi+b_{2} \eta+b_{3} \xi \eta$

where the coefficients $a_{i}$ and $b_{i}$ can be obtained by evaluating these relations for the nodes of the element. For $a_{i}$ s for example: 
$\left[\begin{array}{l}x_{1} \\ x_{2} \\ x_{3} \\ x_{4}\end{array}\right]=\left[\begin{array}{cccc}1 & -1 & -1 & 1 \\ 1 & 1 & -1 & -1 \\ 1 & 1 & 1 & 1 \\ 1 & -1 & 1 & -1\end{array}\right]\left[\begin{array}{l}a_{0} \\ a_{1} \\ a_{2} \\ a_{3}\end{array}\right]$

B- 3

The DFE stiffness matrix obtained in Equation 86, was developed for any arbitrary closed area in element domain. Using relationships between element and natural coordinate systems developed above, differentials of DFE matrix can be replaced with appropriate natural coordinate equivalent terms.

The first integral, for example in Equation 86 is repeated here for explanation of the derivation:

$\oint\left(\frac{\partial w}{\partial x}\right)\left(\frac{\partial^{2} \delta w}{\partial x^{2}}+v \frac{\partial^{2} \delta w}{\partial y^{2}}\right) n_{y} d s$

B- 4

In this integral, the term $n_{y} d s$ will translate to differential $d y$, which is evaluated around the element boundaries in a counter clockwise positive sense. Using the chain rules this differential can be transformed from the element coordinate system to the natural coordinate system:

$d y=\frac{\partial y}{\partial \xi} d \xi+\frac{\partial y}{\partial \eta} d \eta=\left(b_{1}+b_{3} \eta\right) d \xi+\left(b_{2}+b_{3} \xi\right) d \eta$

B- 5

When evaluating the closed loop integrals, the bottom edge in natural coordinate system will have a positive sense for differential $d \xi$, while for the top edge, the positive direction of $\xi$ will correspond to a clockwise rotation, and therefore should be considered with a negative sign. Thus, the top and bottom edge will contribute to the closed loop integral as:

$\int_{-1}^{1}\left[\left(\frac{\partial[w]^{T}}{\partial x}\right)\left(\frac{\partial^{2}[w]}{\partial x^{2}}+v \frac{\partial^{2}[w]}{\partial y^{2}}\right)\left(b_{1}+b_{3} \eta\right)\right]_{\eta=1}^{\eta=-1} d \xi$

B- 6

For the side edges, the positive sense of rotation will be mapped on the right edge of the mapping element, in natural coordinate system, and vice versa. Thus, the remaining edges are converted to the natural coordinate system as:

$\int_{-1}^{1}\left[\left(\frac{\partial[w]^{T}}{\partial x}\right)\left(\frac{\partial^{2}[w]}{\partial x^{2}}+v \frac{\partial^{2}[w]}{\partial y^{2}}\right)\left(b_{2}+b_{3} \xi\right)\right]_{\xi=-1}^{\xi=1} d \eta$

Therefore, the first closed line integral of Equation 86 can be written as summation of two line integrals along opposite edges. If the displacement function can be defined such that the dynamic shape functions are solutions of the Kirchhoff's plate equation in element coordinate system, this integral can be written as follows:

$$
\begin{aligned}
& \int_{-1}^{1}\left[\left(\frac{\partial\left[N_{\omega}\right]^{T}}{\partial x}\right)\left(\frac{\partial^{2}\left[N_{\omega}\right]}{\partial x^{2}}+v \frac{\partial^{2}\left[N_{\omega}\right]}{\partial y^{2}}\right)\left(b_{1}+b_{3} \eta\right)\right]_{\eta=1}^{\eta=-1} d \xi \\
& +\int_{-1}^{1}\left[\left(\frac{\partial\left[N_{\omega}\right]^{T}}{\partial x}\right)\left(\frac{\partial^{2}\left[N_{\omega}\right]}{\partial x^{2}}+v \frac{\partial^{2}\left[N_{\omega}\right]}{\partial y^{2}}\right)\left(b_{2}+b_{3} \xi\right)\right]_{\xi=-1}^{\xi=1} d \eta
\end{aligned}
$$


In the above integrations, the shape function vector, $\left[N_{\omega}\right]$, and its element coordinate system derivatives, must be converted to the natural coordinate system as well, before the integration is performed. This can be done by evaluating the derivatives in the element coordinate system, and then replacing the variables $x$ and $y$ with the respective definitions in natural coordinate system.

Following this approach for converting the remaining integrals, the six line integrals in Equation 86 can be written in natural coordinate system as 12 integrations:

$$
\begin{aligned}
& \frac{\left[K_{\omega}\right]}{D}=\int_{-1}^{1}\left[\left(\frac{\partial\left[N_{\omega}\right]^{T}}{\partial x}\right)\left(\frac{\partial^{2}\left[N_{\omega}\right]}{\partial x^{2}}+v \frac{\partial^{2}\left[N_{\omega}\right]}{\partial y^{2}}\right)\left(b_{1}+b_{3} \eta\right)\right]_{\eta=1}^{\eta=-1} d \xi \\
& +\int_{-1}^{1}\left[\left(\frac{\partial\left[N_{\omega}\right]^{T}}{\partial x}\right)\left(\frac{\partial^{2}\left[N_{\omega}\right]}{\partial x^{2}}+v \frac{\partial^{2}\left[N_{\omega}\right]}{\partial y^{2}}\right)\left(b_{2}+b_{3} \xi\right)\right]_{\xi=-1}^{\xi=1} d \eta \\
& -\int_{-1}^{1}\left[\left(\left(\frac{\partial\left[N_{\omega}^{T}\right]}{\partial y}\right)\left(\frac{\partial^{2}\left[\delta N_{\omega}\right]}{\partial y^{2}}+v \frac{\partial^{2}\left[\delta N_{\omega}\right]}{\partial x^{2}}\right)\right)\left(a_{1}+a_{3} \eta\right)\right]_{\eta=1}^{\eta=-1} d \xi \\
& -\int_{-1}^{1}\left[\left(\left(\frac{\partial\left[N_{\omega}^{T}\right]}{\partial y}\right)\left(\frac{\partial^{2}\left[\delta N_{\omega}\right]}{\partial y^{2}}+v \frac{\partial^{2}\left[\delta N_{\omega}\right]}{\partial x^{2}}\right)\right)\left(a_{2}+a_{3} \xi\right)\right]_{\xi=-1}^{\xi=1} d \eta \\
& \left.+\int_{-1}^{1}\left[\left((1-v) \frac{\partial\left[N_{\omega}\right]^{T}}{\partial y}\right) \frac{\partial^{2} \delta\left[N_{\omega}\right]}{\partial x \partial y}\right)\left(b_{1}+b_{3} \eta\right)\right]_{\eta=1}^{\eta=-1} d \xi \\
& \left.+\int_{-1}^{1}\left[\left((1-v) \frac{\partial\left[N_{\omega}\right]^{T}}{\partial y}\right) \frac{\partial^{2} \delta\left[N_{\omega}\right]}{\partial x \partial y}\right)\left(b_{2}+b_{3} \xi\right)\right]_{\xi=-1}^{\xi=1} d \eta \\
& -\int_{-1}^{1}\left[\left(\left((1-v) \frac{\partial\left[N_{\omega}^{T}\right]}{\partial x}\right) \frac{\partial^{2}\left[\delta N_{\omega}\right]}{\partial x \partial y}\right)\left(a_{1}+a_{3} \eta\right)\right]_{\eta=1}^{\eta=-1} d \xi \\
& +\int_{-1}^{1}\left[\left(\left((1-v) \frac{\partial\left[N_{\omega}^{T}\right]}{\partial x}\right) \frac{\partial^{2}\left[\delta N_{\omega}\right]}{\partial x \partial y}\right)\left(a_{2}+a_{3} \xi\right)\right]_{\xi=-1}^{\xi=1} d \eta \\
& -\int_{-1}^{1}\left[\left(\left[N_{\omega}^{T}\right]\right)\left(\frac{\partial^{3}\left[\delta N_{\omega}\right]}{\partial x^{3}}+\frac{\partial^{3}\left[\delta N_{\omega}\right]}{\partial x \partial y^{2}}\right)\left(b_{2}+b_{3} \xi\right)\right]_{\xi=-1}^{\xi=1} d \eta \\
& \left.-\int_{-1}^{1}\left[\left(\left[N_{\omega}^{T}\right]\right)\left(\frac{\partial^{3}\left[\delta N_{\omega}\right]}{\partial x^{3}}+\frac{\partial^{3}\left[\delta N_{\omega}\right]}{\partial x \partial y^{2}}\right)\right)\left(b_{1}+b_{3} \eta\right)\right]_{\eta=1}^{\eta=-1} d \xi \\
& \eta=-1
\end{aligned}
$$


$+\int_{-1}^{1}\left[\left(\left(\left[N_{\omega}^{T}\right]\right)\left(\frac{\partial^{3}\left[\delta N_{\omega}\right]}{\partial y^{3}}+\frac{\partial^{3}\left[\delta N_{\omega}\right]}{\partial y \partial x^{2}}\right)\right)\left(a_{2}+a_{3} \xi\right)\right]_{\xi=-1}^{\xi=1} d \eta$

It is important to note that the relationship between the natural and element coordinate system does not need to be bi-linear, and neither the geometry is restricted to quadrilaterals. The same procedure is applicable to any geometry and any relationship, as long as a mapping to the square reference geometry in natural coordinate system is obtainable. For example, a four-node element with curved edges can be mapped to natural coordinate system using quadrilateral or cubic relationships.

The area integrals in Equation 86 were equated to zero in development of plate DFE. This reduction was possible for shape functions that were obtained from solutions of the governing differential equation of the plate. As such, for plates of arbitrary shapes, the shape functions must be defined in element coordinate system and then transformed to the natural coordinate system using variable substitution.

Since Equation 86 was a mathematical manipulation of Galerkin's symmetric FEM formulation in Equation 53, it will be symmetric as well and area integrals can be eliminated. On the other hand, if the shape functions used are not solution of plate equation, such eliminations will not be possible, and mapping will involve area integral transformations of Equation 53 to natural coordinate system, using Jacobian matrix. Since DFE shape functions are generally transcendental functions of natural frequency of vibration, the new mapped integrals are analytically difficult to evaluate and numerically error prone to calculate. 


\section{APPENDIX C}

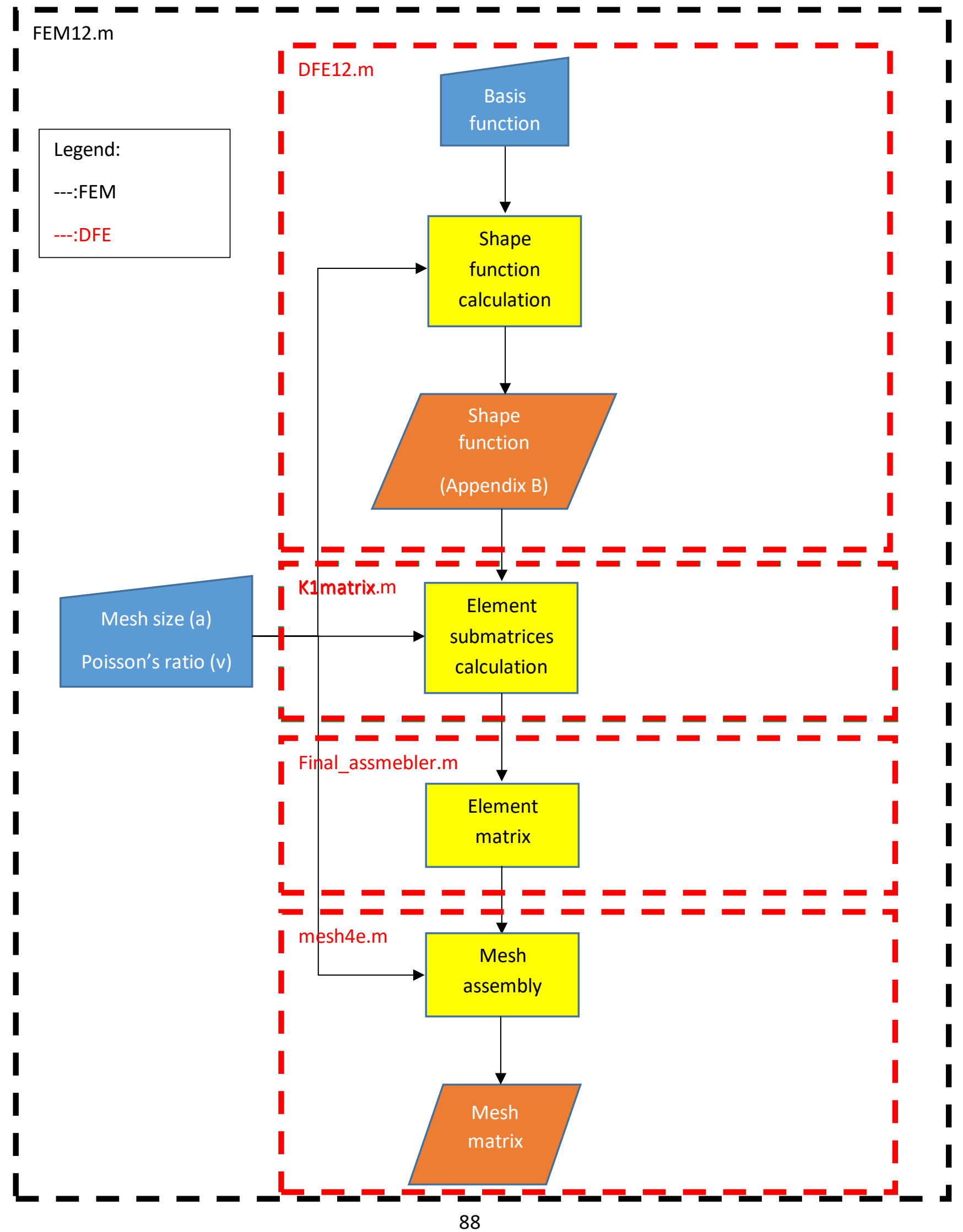




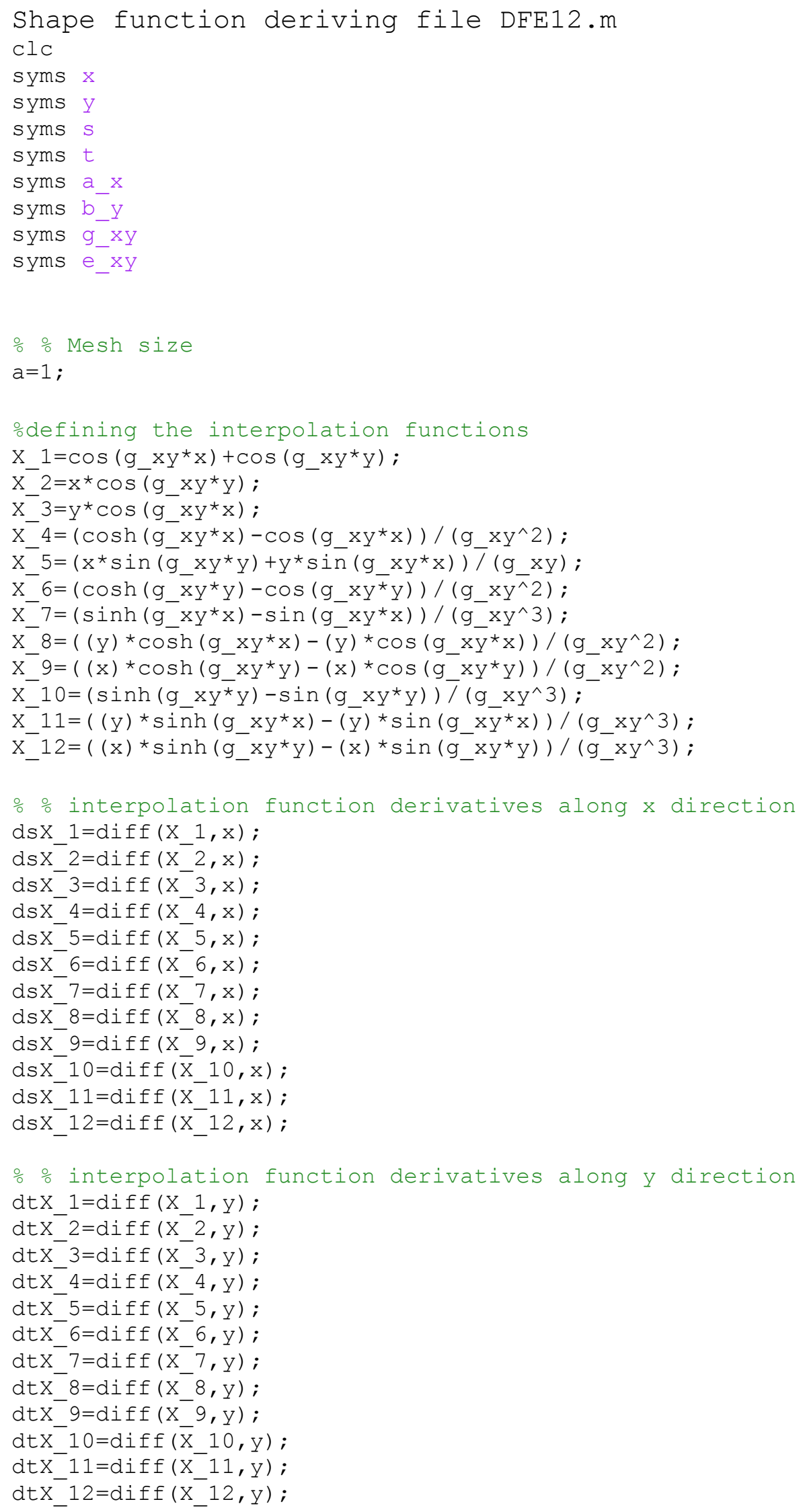




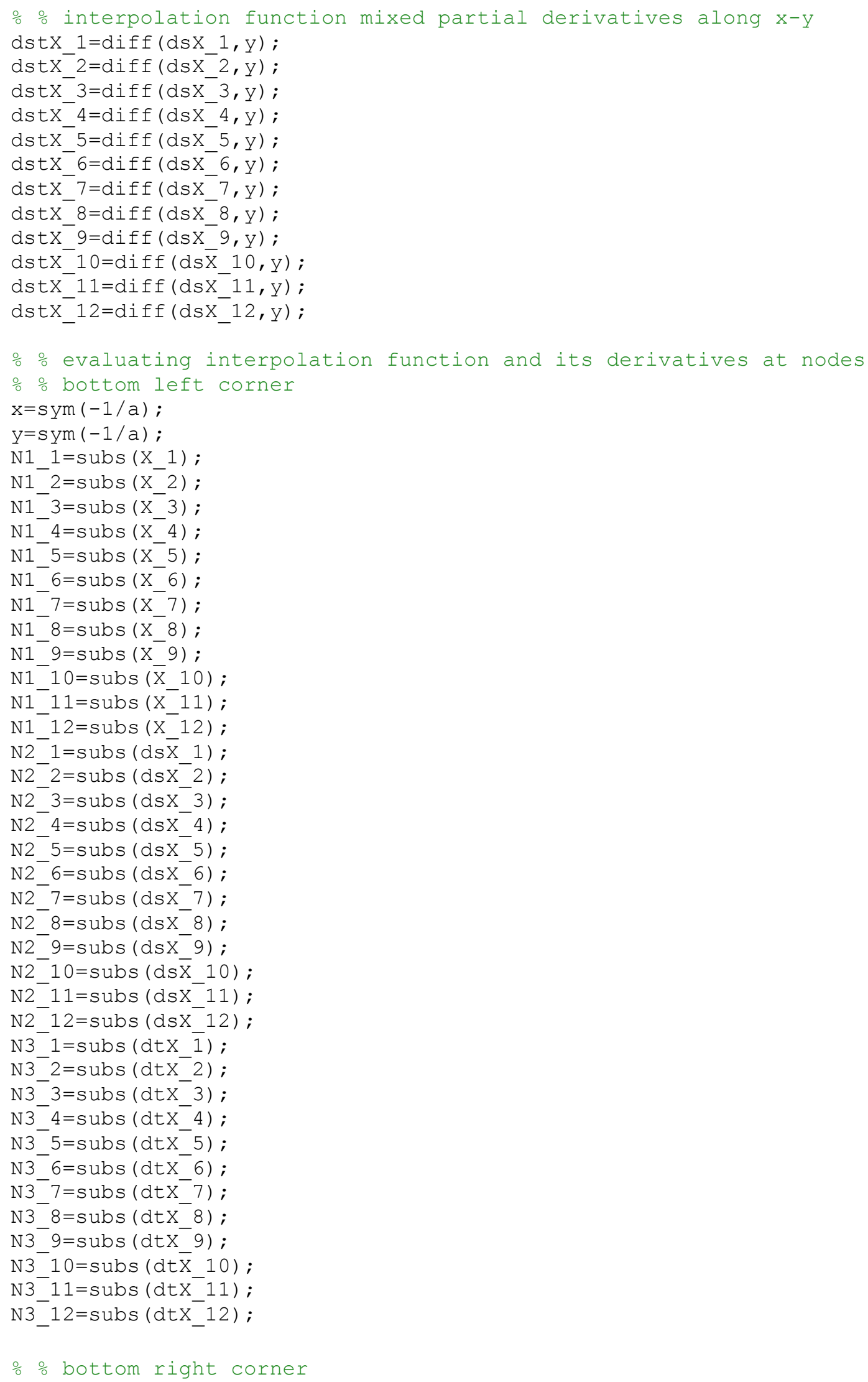




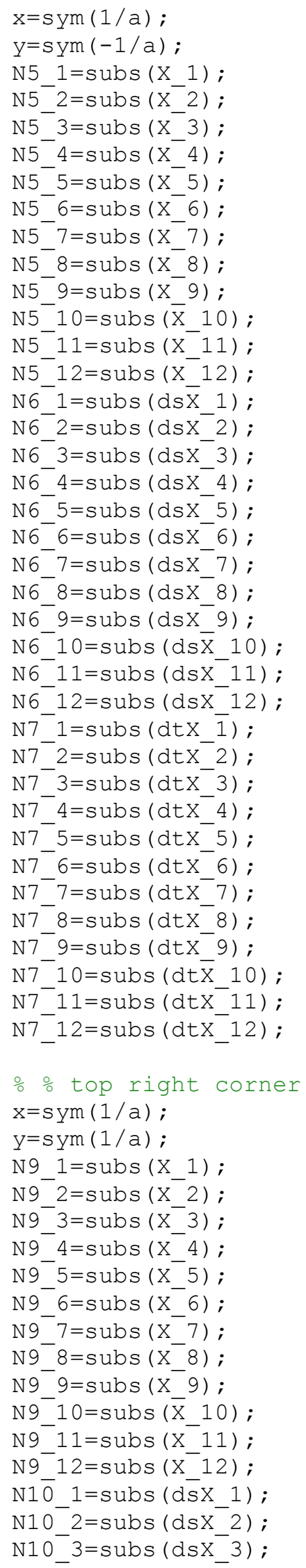




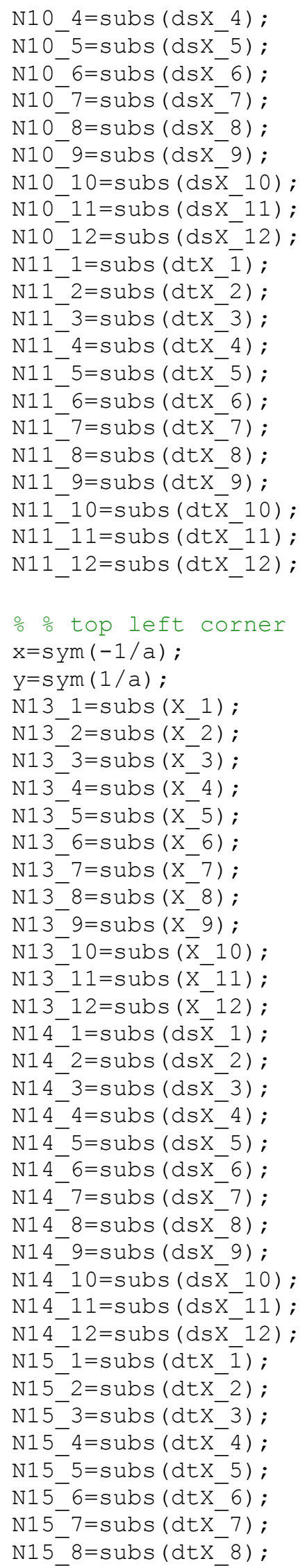




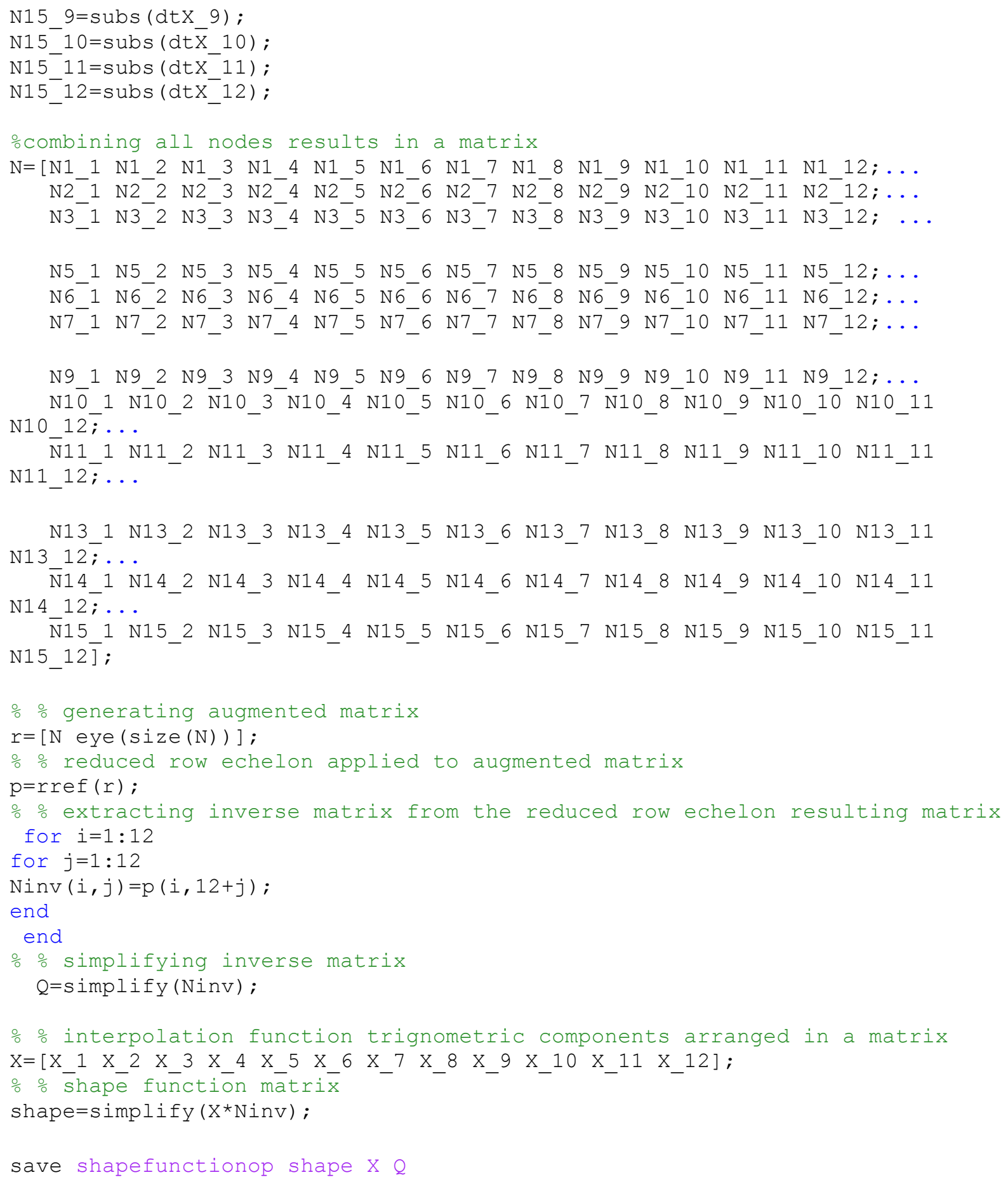


First DFE submatrix file deriving file Klmatrix.m (Similar files are developed for 9 other DFE integrations but are omitted here for brevity)

clear

$\mathrm{clc}$

syms $x$

syms $y$

syms g_xy

응 Mesh size

$\mathrm{a}=1$;

응 loading the shape function

load shapefunctionop

응 applying variable constraints for easier evaluation and reduced compitation time

assume ( $\mathrm{x}$, 'real')

assume ( $y$, 'real')

assume (g_xy,'real')

assume (9_xy>0)

\% generating derivatives vectors

$\operatorname{Diffl}=\operatorname{simplify}(\operatorname{diff}(X, x))$;

Diff2=simplify $(\operatorname{diff}(X, y))$;

Diff $3=\operatorname{simplify}(\operatorname{diff}(X, x, 2))$;

Diff4=simplify $(\operatorname{diff}(\operatorname{diff}(X, x), y))$;

Diff5=simplify $(\operatorname{diff}(X, Y, 2))$;

Diff $6=\operatorname{simplify}(\operatorname{diff}(X, x, 3))$;

Diff7=simplify $(\operatorname{diff}(\operatorname{diff}(X, x, 2), y))$;

Diff $8=\operatorname{simplify}(\operatorname{diff}(\operatorname{diff}(X, x), y, 2))$;

Diff9=simplify (diff $(X, Y, 3))$;

Diff_total=[Diff1;Diff2;Diff3;Diff4;Diff5;Diff6;Diff7;Diff8;Diff9];

\% calculating the first DEE matrix

$k 1=$ transpose $(\operatorname{Diff} 1) * \operatorname{Diff} 3$;

$\mathrm{k} 1 \mathrm{~s}=(\operatorname{subs}(\mathrm{k} 1, \mathrm{x}, \operatorname{sym}(1 / \mathrm{a}))-\operatorname{subs}(\mathrm{k} 1, \mathrm{x}, \operatorname{sym}(-1 / \mathrm{a})))$;

$\mathrm{k} 11=\mathrm{int}(\mathrm{k} 1 \mathrm{~s}, \mathrm{y})$;

$\mathrm{K} 1=\operatorname{subs}(k 11, y, \operatorname{sym}(1 / a))-\operatorname{subs}(k 11, y, \operatorname{sym}(-1 / a))$;

\% 응 simplifying the final form of first DFE matrix

K1_f=simplify(K1,' IgnoreAnalyticConstraints',true);

save KImatrix_f $K I_{-} f$ 


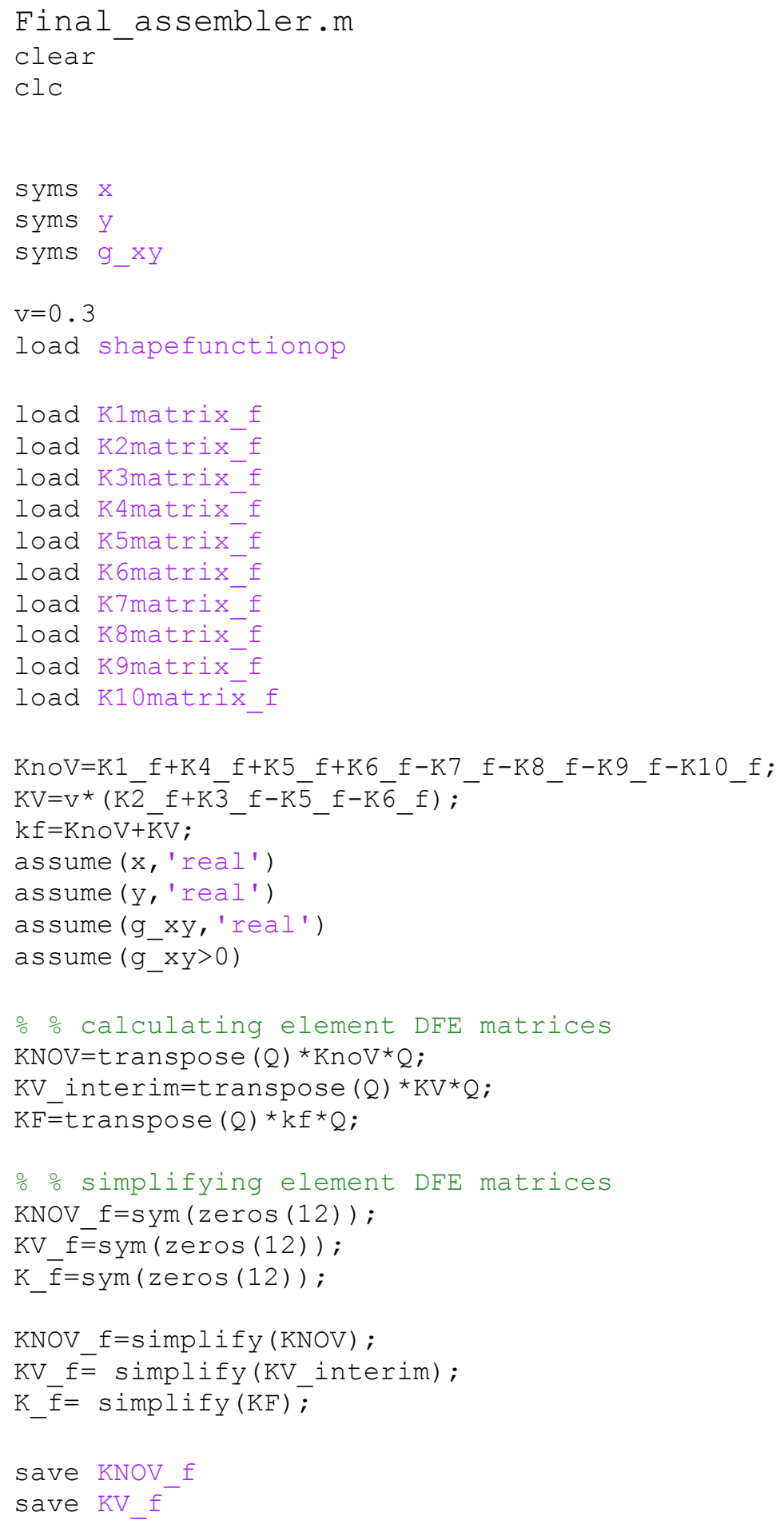


4 element DFE mesh assembly file mesh4e.m (Similar files for other emsh sizes are developed but are not presented here for brevity. See FEM12 for reference)

K_4e=sym $(\operatorname{zeros}(27))$

bottom left element

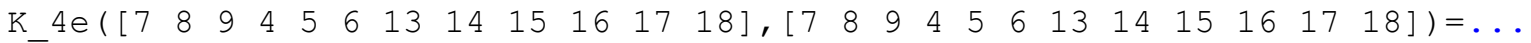
K 4 e $\left(\left[\begin{array}{llllllllllll}7 & 8 & 9 & 4 & 5 & 6 & 13 & 14 & 15 & 16 & 17 & 18\end{array}\right],\left[\begin{array}{lllllllllll}7 & 8 & 9 & 4 & 5 & 6 & 13 & 14 & 15 & 16 & 17\end{array}\right.\right.$

18] ) $+\overline{\mathrm{K}} \_$f ;

\% bottom right element

$K_{-} 4 e\left(\left[\begin{array}{llllllllllll}4 & 5 & 6 & 1 & 2 & 3 & 10 & 11 & 12 & 13 & 14 & 15\end{array}\right],\left[\begin{array}{llllllllllll}4 & 5 & 6 & 1 & 2 & 3 & 10 & 11 & 12 & 13 & 14 & 15\end{array}\right]\right)=\ldots$ $\mathrm{K} 4 \mathrm{e}\left(\left[\begin{array}{lllllllllllll}4 & 5 & 6 & 1 & 2 & 3 & 10 & 11 & 12 & 13 & 14 & 15\end{array}\right],\left[\begin{array}{lllllllllll}4 & 5 & 6 & 1 & 2 & 3 & 10 & 11 & 12 & 13 & 14\end{array}\right.\right.$

15]) $+\overline{\mathrm{K}}_{-} \mathrm{f}$;

top right element

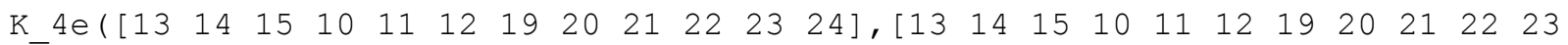
$24])=\ldots$ K $4 \mathrm{e}\left(\left[\begin{array}{llllllllllll}13 & 14 & 15 & 10 & 11 & 12 & 19 & 20 & 21 & 22 & 23 & 24\end{array}\right],\left[\begin{array}{lllllllllll}13 & 14 & 15 & 10 & 11 & 12 & 19 & 20 & 21 & 22\end{array}\right.\right.$ $2324 \overline{]})+K_{-} f$;

\% top left element

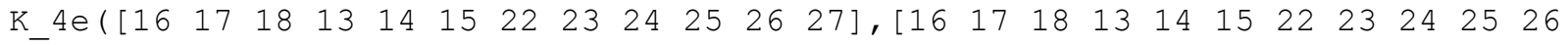
$2 \overline{7}])=\ldots$

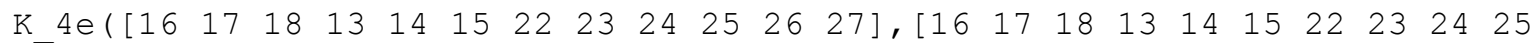
26 27 $\overline{]})+K_{-} f$; 
FEM analysis file FEM12.m

clc

syms $x$

syms y

syms $s$

syms $t$

syms g_xy

syms g_xy4

$a=1$;

$\mathrm{V}=0.3 ;$

odefining the interpolation functions

$\mathrm{x} 1=1$;

$\mathrm{x}_{-} 2=\mathrm{x} ;$

$\mathrm{x}-3=\mathrm{y} ;$

$x \quad 4=x^{\wedge} 2$;

$x_{-}^{-} 5=x^{*} y ;$

$\mathrm{x}_{-} 6=\mathrm{y}^{\wedge} 2$;

$\mathrm{x}_{-}^{-} 7=\mathrm{x}^{\wedge} 3$;

$\mathrm{x}-8=\mathrm{x}^{\wedge} 2^{*} \mathrm{y}$;

$\mathrm{x}_{-}^{-} 9=\mathrm{x}^{*} \mathrm{y}^{\wedge} 2$;

$\mathrm{x}_{-}^{-10} 10 \mathrm{y}^{\wedge} 3$;

$\mathrm{x}_{-}^{-11=x^{\wedge} 3^{*} \mathrm{y} ;}$

$\mathrm{x}_{-}^{-12}=\mathrm{x}^{*} \mathrm{y}^{\wedge} 3$;

응 interpolation function derivatives along $x$ direction

dsX_1=diff $\left(X_{-} 1, x\right)$;

ds $X_{-} 2=\operatorname{diff}\left(\mathrm{X}_{-}^{-} 2, \mathrm{x}\right)$;

dsX_3=diff $\left(X_{-}^{-} 3, x\right)$;

dsX_4=diff $\left(X_{-}^{-} 4, x\right)$;

dsX_5=diff $\left(X^{-} 5, x\right)$;

ds $X_{-}^{-6}=\operatorname{diff}\left(X_{-}^{-} 6, x\right)$;

ds $X_{-}^{-} 7=\operatorname{diff}\left(X_{-}^{-} 7, x\right)$;

dsX_8 $=$ diff $\left(X_{-} 8, x^{\prime}\right)$;

dsX_9=diff $\left(X_{-}^{-} 9, x\right)$;

$\operatorname{ds} X_{-} 10=\operatorname{diff}\left(\bar{X}_{-} 10, x\right)$;

ds $X_{-11}^{-}$diff $\left(X_{-}^{-11, x}\right)$;

dsX_12=diff $\left(X_{-}^{-} 12, x\right)$;

\% $\frac{\circ}{0}$ interpolation function derivatives along $y$ direction

$\operatorname{dtx} \mathrm{X}_{-}=\operatorname{diff}\left(\mathrm{X} \_1, \mathrm{y}\right)$;

$\operatorname{dtX} 2=\operatorname{diff}\left(\mathrm{X}_{-}^{-} 2, \mathrm{Y}\right)$;

$\operatorname{dtx} 3=\operatorname{diff}\left(\mathrm{X}_{-} 3, \mathrm{y}\right)$;

dtx_4=diff $\left(X_{-}^{-} 4, y\right)$;

dtX_5=diff $(X-5, y)$;

dtX_6=diff $\left(X_{-}-6, y\right)$;

$\operatorname{dtx} \mathrm{X}_{-}^{-}=\operatorname{diff}\left(\mathrm{X}_{-}^{-} 7, \mathrm{y}\right)$;

$\operatorname{dtx} \mathrm{X}_{-} 8=\operatorname{diff}\left(\mathrm{X}_{-}^{-} 8, \mathrm{Y}\right)$;

dtX_9=diff $\left(X_{-}\right.$9, y) ;

dtX_10=diff $\left(\bar{X}_{-} 10, y\right)$;

$\operatorname{dtx} \mathrm{X}_{-11}=\operatorname{diff}\left(\mathrm{X}_{-}^{-11}, \mathrm{y}\right)$;

$\operatorname{dtx}{ }_{-}^{-12}=\operatorname{diff}\left(X_{-}^{-} 12, y\right)$; 


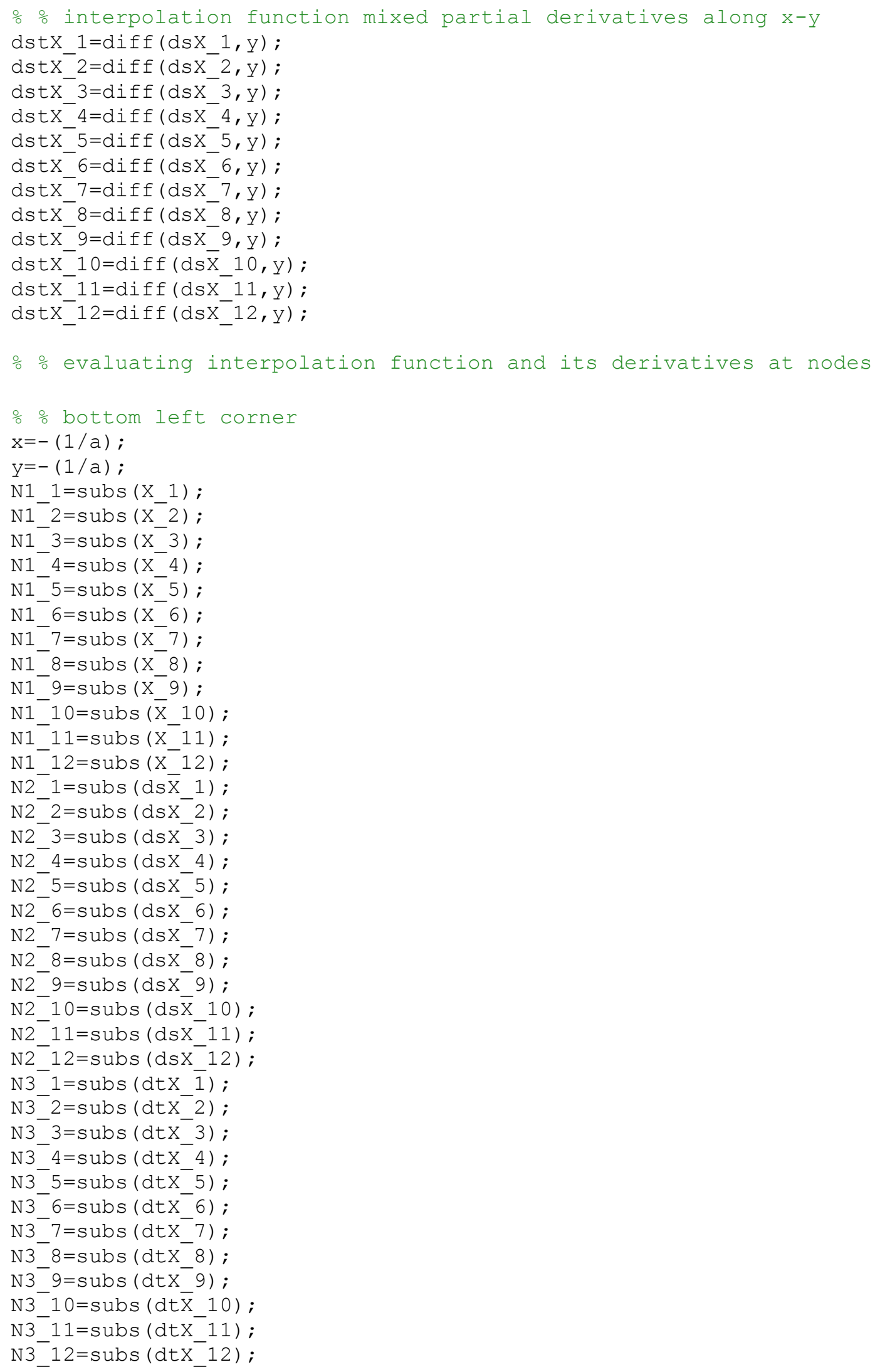




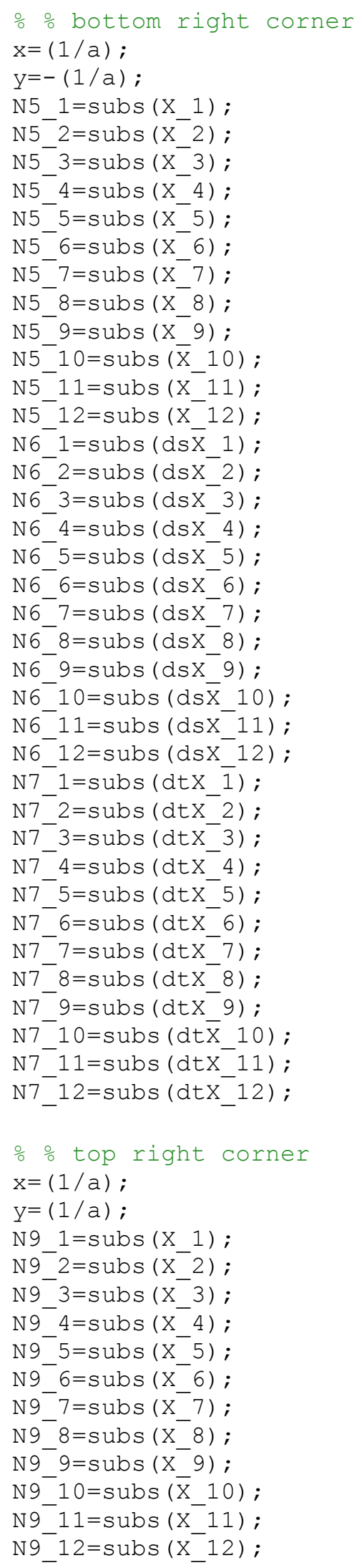




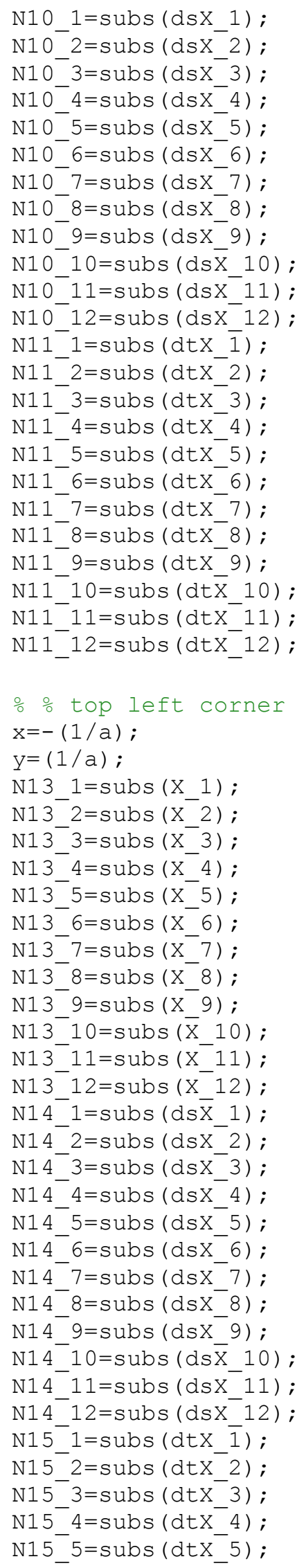




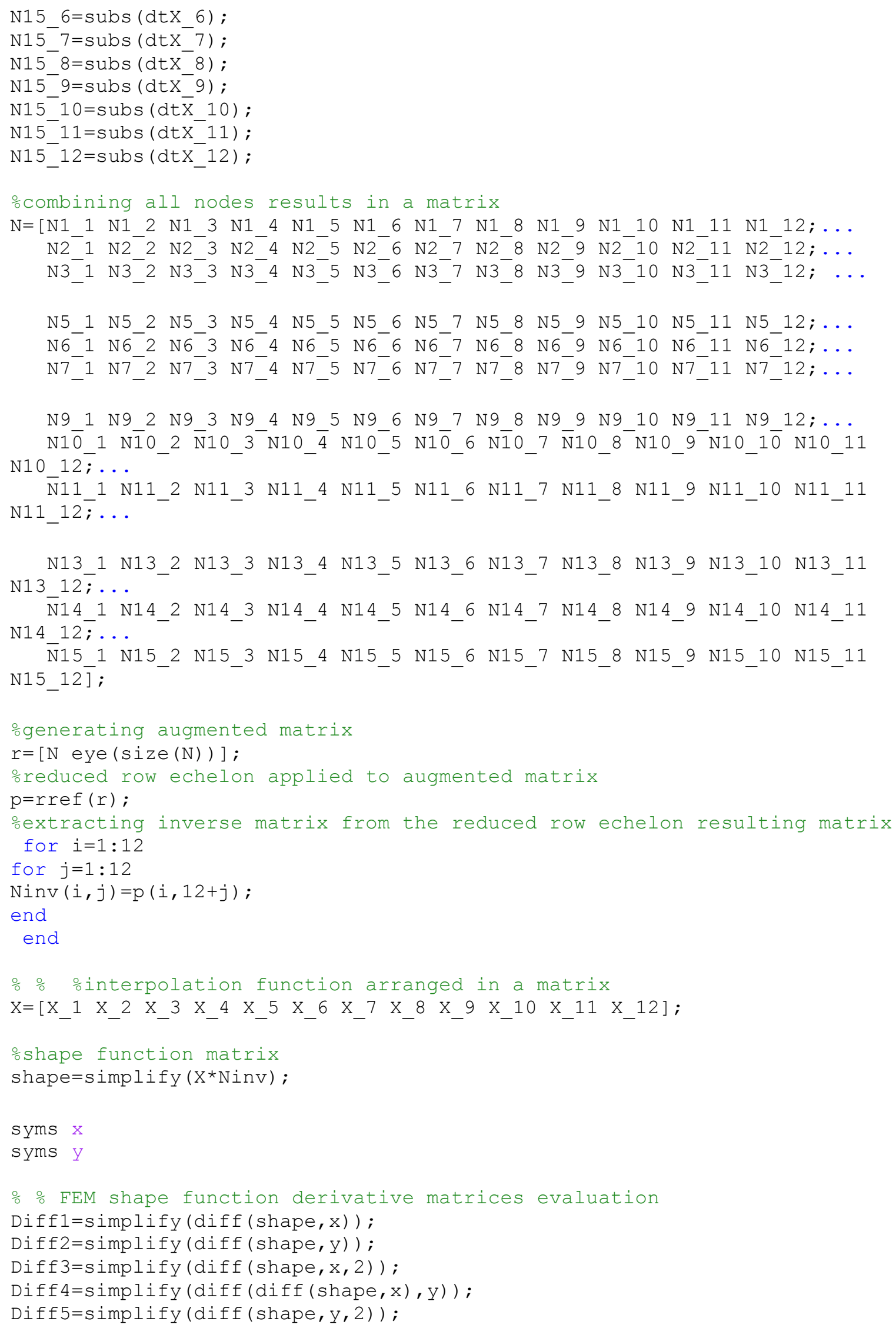




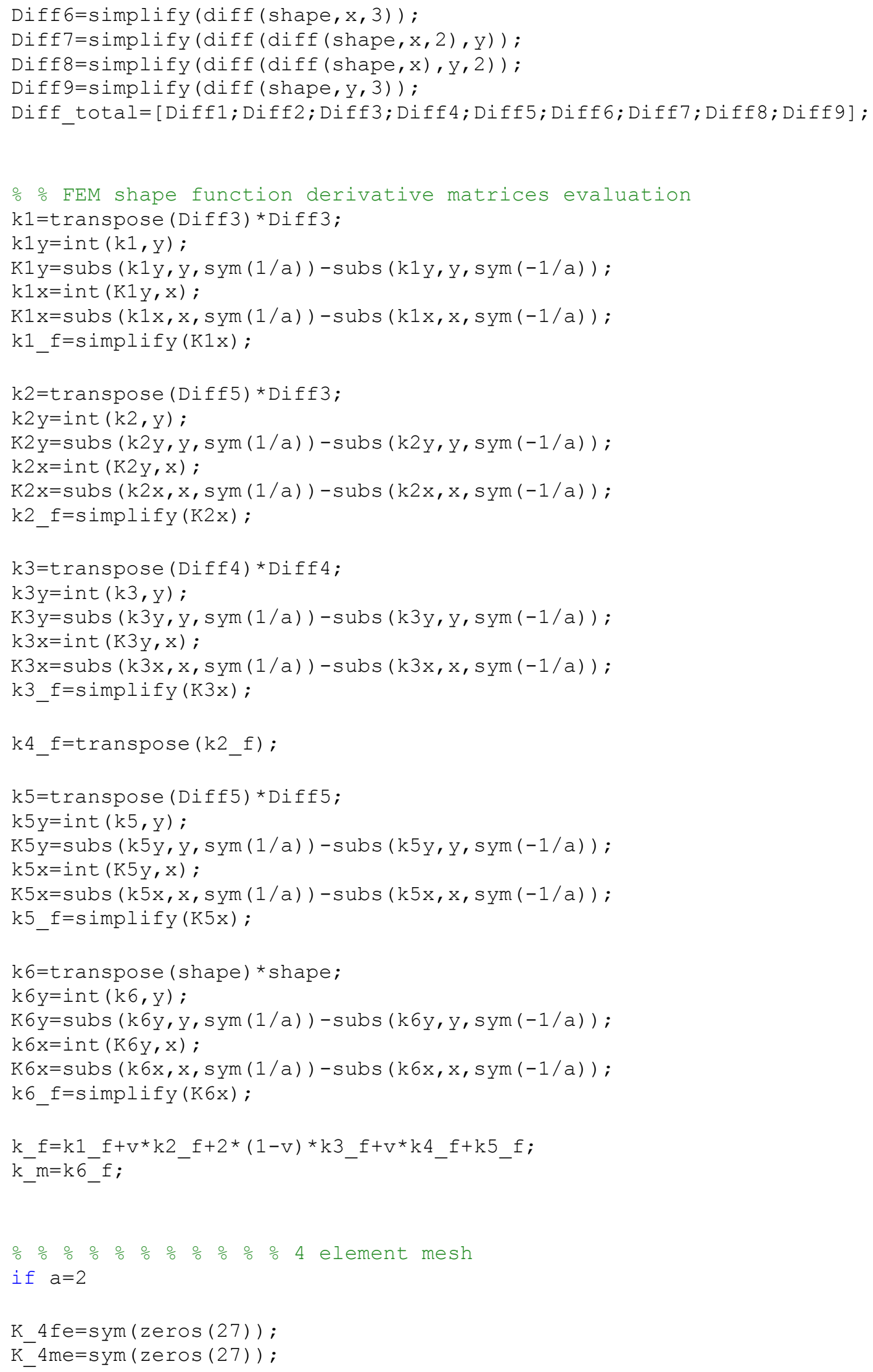




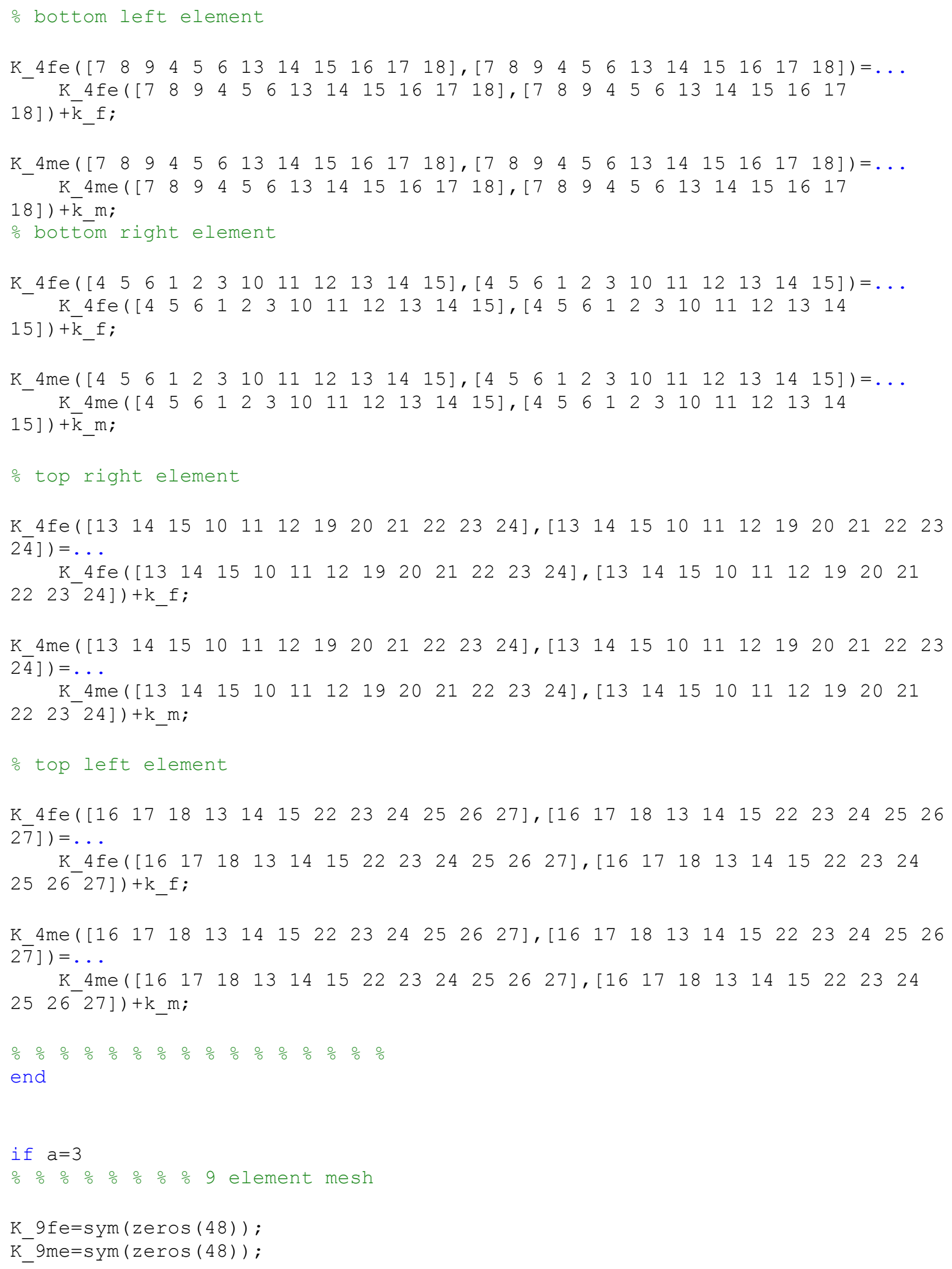


응 bottom left element

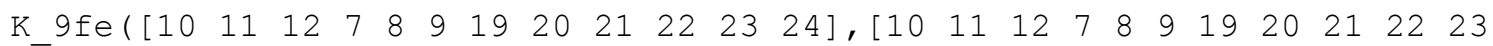

$2 \overline{4}])=\ldots$

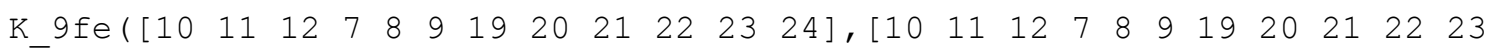
24]) $+\overline{\mathrm{k}} \_$f ;

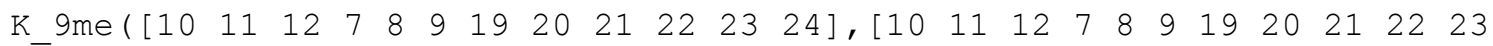

$2 \overline{4}])=\ldots$

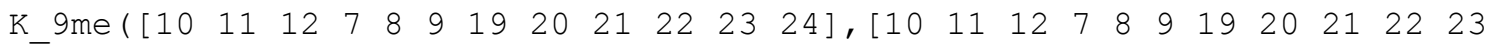
24] ) $+\overline{\mathrm{k}} \mathrm{m}$;

응 bottom middle element

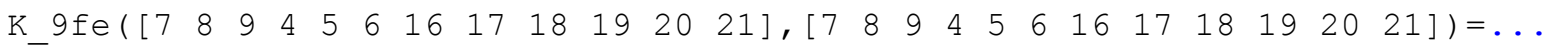
21]) $+\bar{k} \_$f ;

K_9me $\left(\left[\begin{array}{lllllllllllll}7 & 8 & 9 & 4 & 5 & 6 & 16 & 17 & 18 & 19 & 20 & 21\end{array}\right],\left[\begin{array}{llllllllllll}7 & 8 & 9 & 4 & 5 & 6 & 16 & 17 & 18 & 19 & 20 & 21\end{array}\right)=\ldots\right.$ K_9me $\left(\left[\begin{array}{llllllllllll}7 & 8 & 9 & 4 & 5 & 6 & 16 & 17 & 18 & 19 & 20 & 21\end{array}\right],\left[\begin{array}{lllllllllll}7 & 8 & 9 & 4 & 5 & 6 & 16 & 17 & 18 & 19 & 20\end{array}\right.\right.$

21] ) $+\overline{\mathrm{k}} \mathrm{m}$;

응 bottom right element

K_9fe([ [ $\left.\begin{array}{llllllllllll}4 & 5 & 6 & 1 & 2 & 3 & 13 & 14 & 15 & 16 & 17 & 18\end{array}\right],\left[\begin{array}{llllllllllll}4 & 5 & 6 & 1 & 2 & 3 & 13 & 14 & 15 & 16 & 17 & 18\end{array}\right)=\ldots$ K_9fe $\left(\left[\begin{array}{llllllllllll}4 & 5 & 6 & 1 & 2 & 3 & 13 & 14 & 15 & 16 & 17 & 18\end{array}\right],\left[\begin{array}{lllllllllll}4 & 5 & 6 & 1 & 2 & 3 & 13 & 14 & 15 & 16 & 17\end{array}\right.\right.$

$18])+\bar{k} \_$f ;

K_9me $\left(\left[\begin{array}{lllllllllllll}4 & 5 & 6 & 1 & 2 & 3 & 13 & 14 & 15 & 16 & 17 & 18\end{array}\right],\left[\begin{array}{llllllllllll}4 & 5 & 6 & 1 & 2 & 3 & 13 & 14 & 15 & 16 & 17 & 18\end{array}\right)=\ldots\right.$

K_9me $\left(\left[\begin{array}{llllllllllll}4 & 5 & 6 & 1 & 2 & 3 & 13 & 14 & 15 & 16 & 17 & 18\end{array}\right],\left[\begin{array}{lllllllllll}4 & 5 & 6 & 1 & 2 & 3 & 13 & 14 & 15 & 16 & 17\end{array}\right.\right.$

18] ) $+\overline{\mathrm{k}} \mathrm{m}$;

응 mi ddle left element

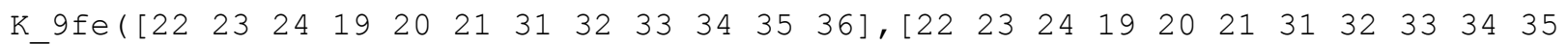
$3 \overline{6}])=\ldots$

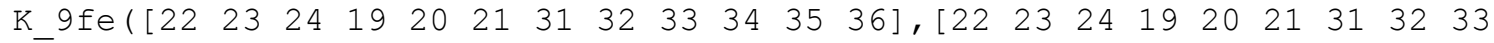

3435 36]) +k_f;

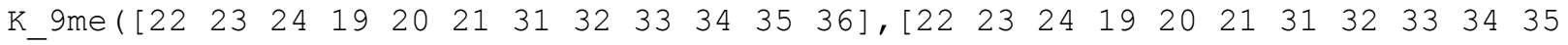
$3 \overline{6}])=\ldots$

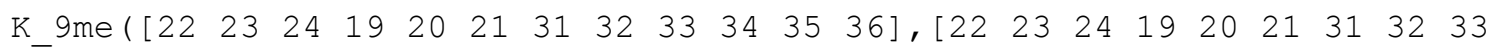

3435 36]) + k_m;

\% middle míddle element

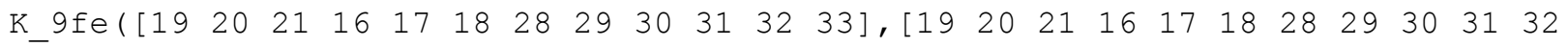
$3 \overline{3}])=\ldots$

K_9fe([l] $\left.\begin{array}{llllllllllll}19 & 20 & 21 & 16 & 17 & 18 & 28 & 29 & 30 & 31 & 32 & 33\end{array}\right],\left[\begin{array}{llllllllll}19 & 20 & 21 & 16 & 17 & 18 & 28 & 29 & 30\end{array}\right.$

3132 33]) +k_f ;

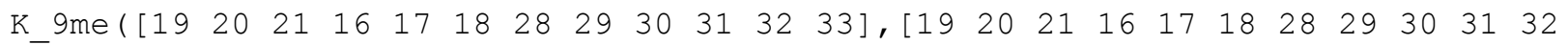
$3 \overline{3}])=\ldots$

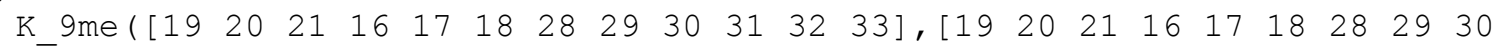

$31 \quad 32$ 33]) +k_m;

\% middle right element

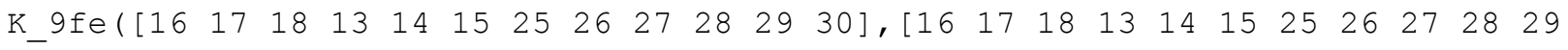
$3 \overline{0}])=\ldots$ 


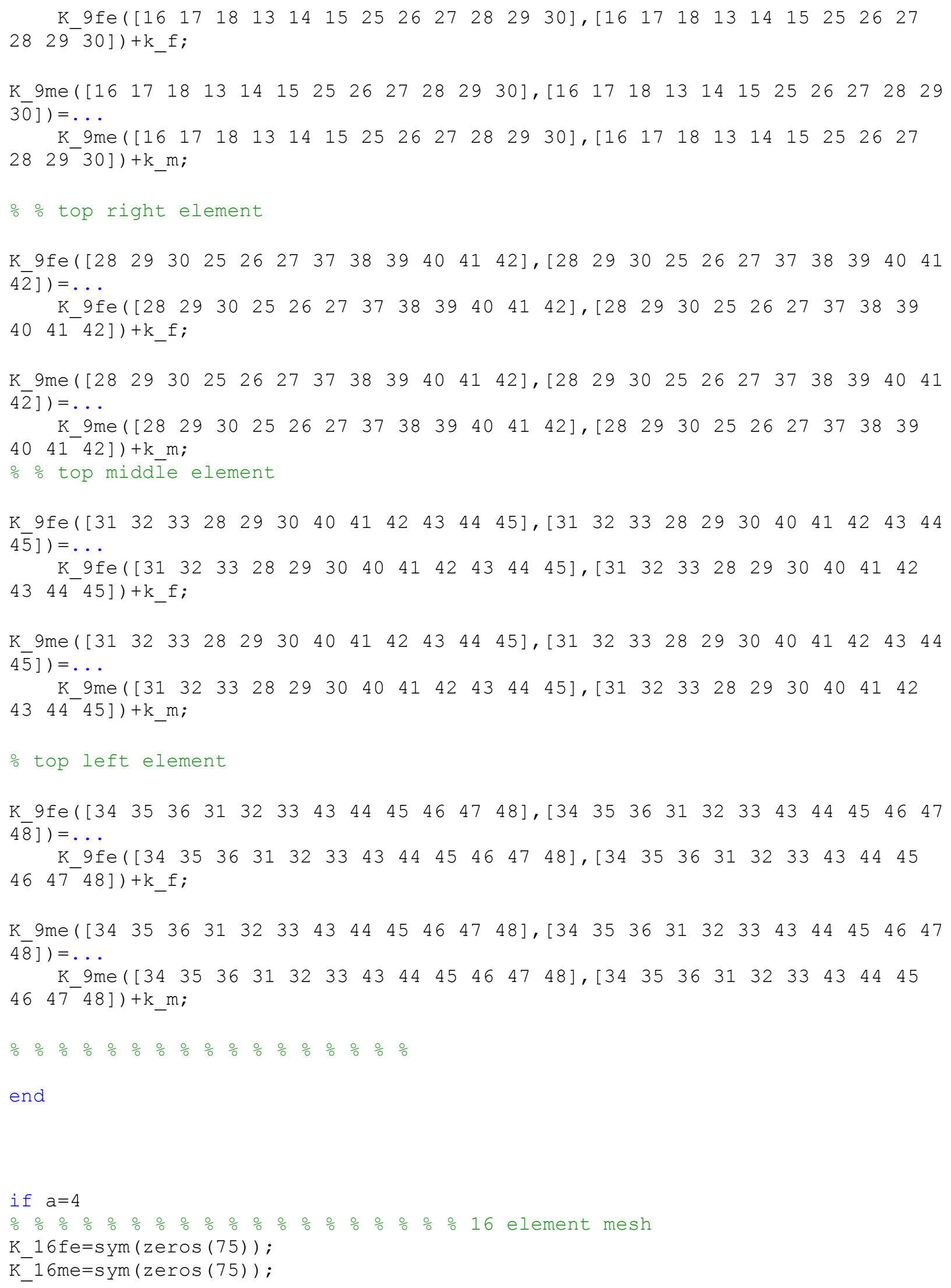




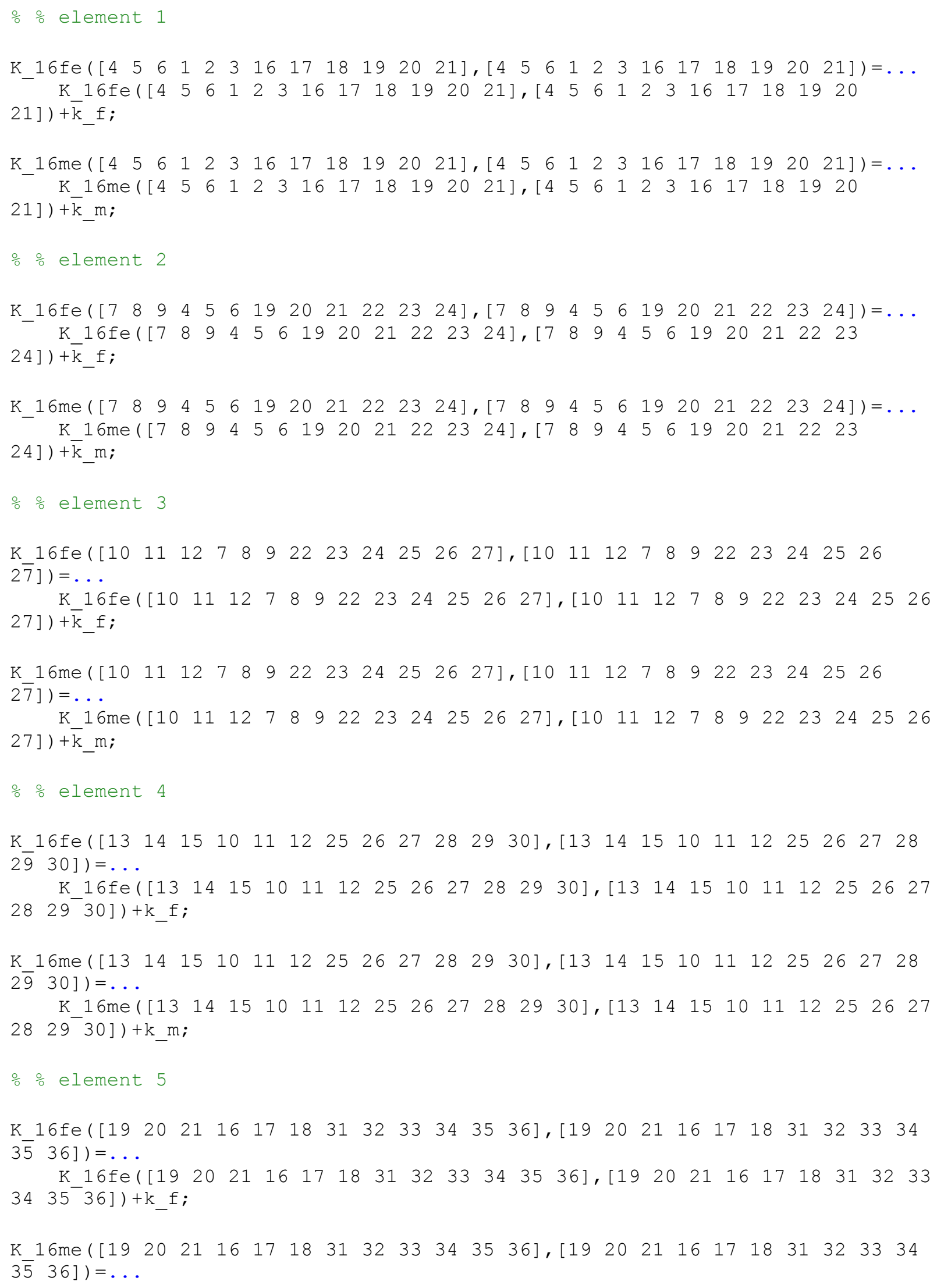




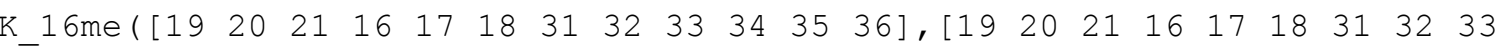
$\left.\left.3435^{-} 36\right]\right)+k$

응 element 6

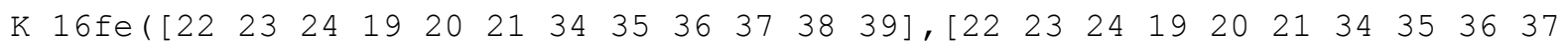

$3 \overline{8} 39])=\ldots$

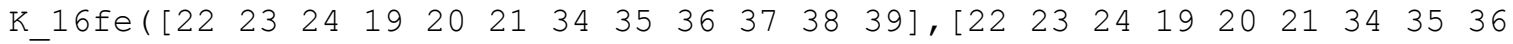

3738 39]) + k_f ;

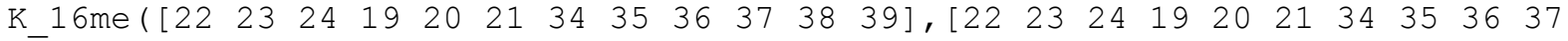
$3 \overline{8} 39])=\ldots$

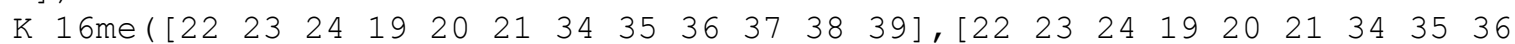

3738 39]) + k_m;

응 응 element 7

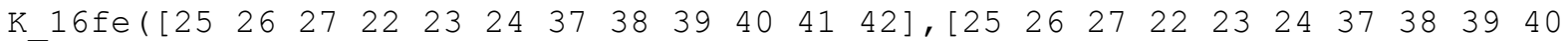

$4 \overline{1} 42])=\ldots$

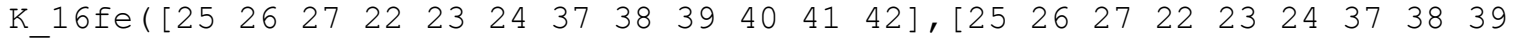
$\left.\left.4041^{-} 42\right]\right)+k_{-}$;

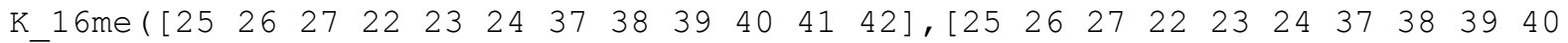
$4 \overline{1} 42])=\ldots$

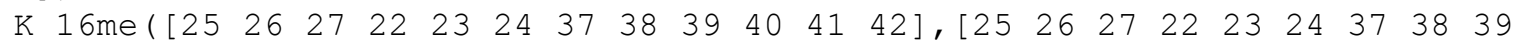

$40 \quad 41-42])+k \_m$;

응 element 8

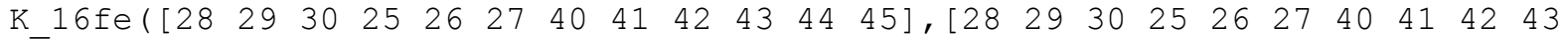

$4 \overline{4} 45])=\ldots$

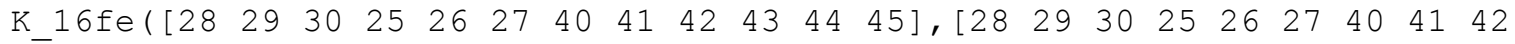
$43 \quad 44-45])+k_{-}$;

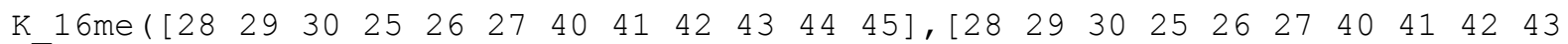

$4 \overline{4} 45])=\ldots$

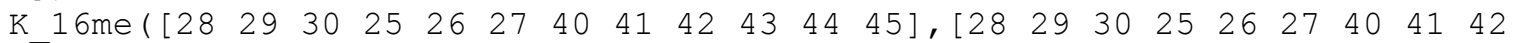

$43 \quad 44\left[\begin{array}{ll}-1 & 4\end{array}\right]+k_{-} m$;

응 element 9

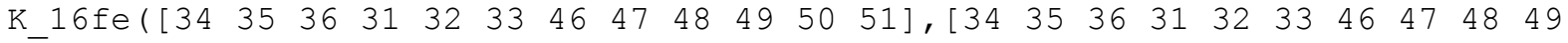

$5 \overline{0} 51])=\ldots$

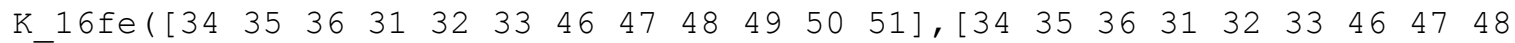
$\left.\left.4950^{-} 51\right]\right)+k_{-}$;

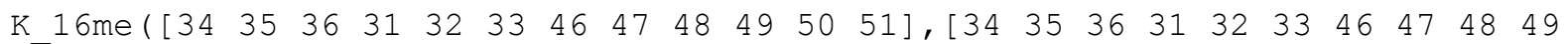
$5 \overline{0} 51])=\ldots$

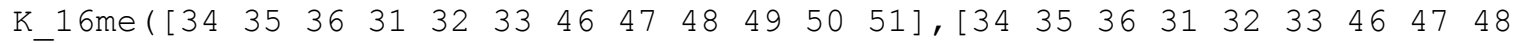
$\left.\left.4950^{-} 51\right]\right)+k_{-}$;

\% element 10 


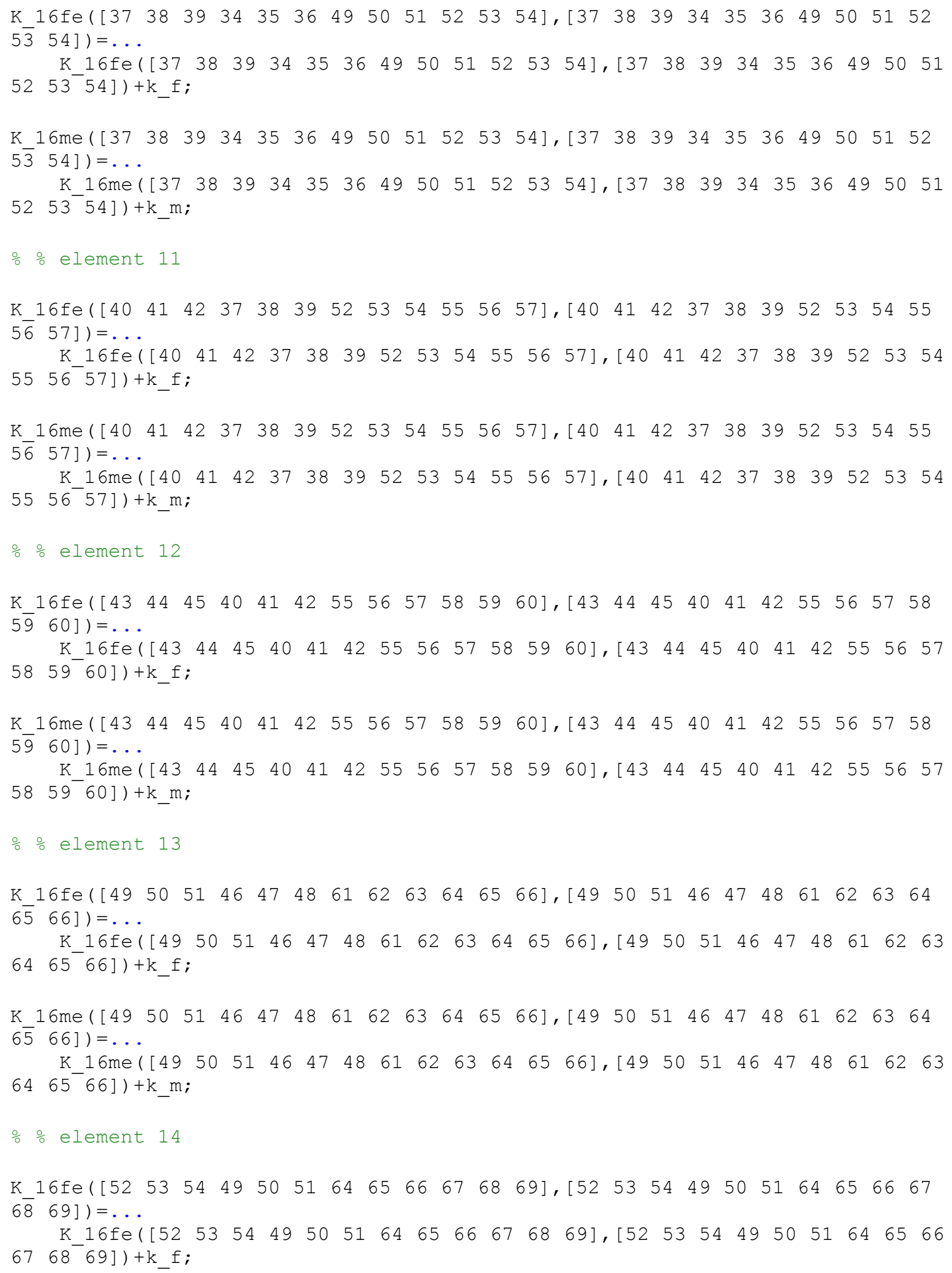




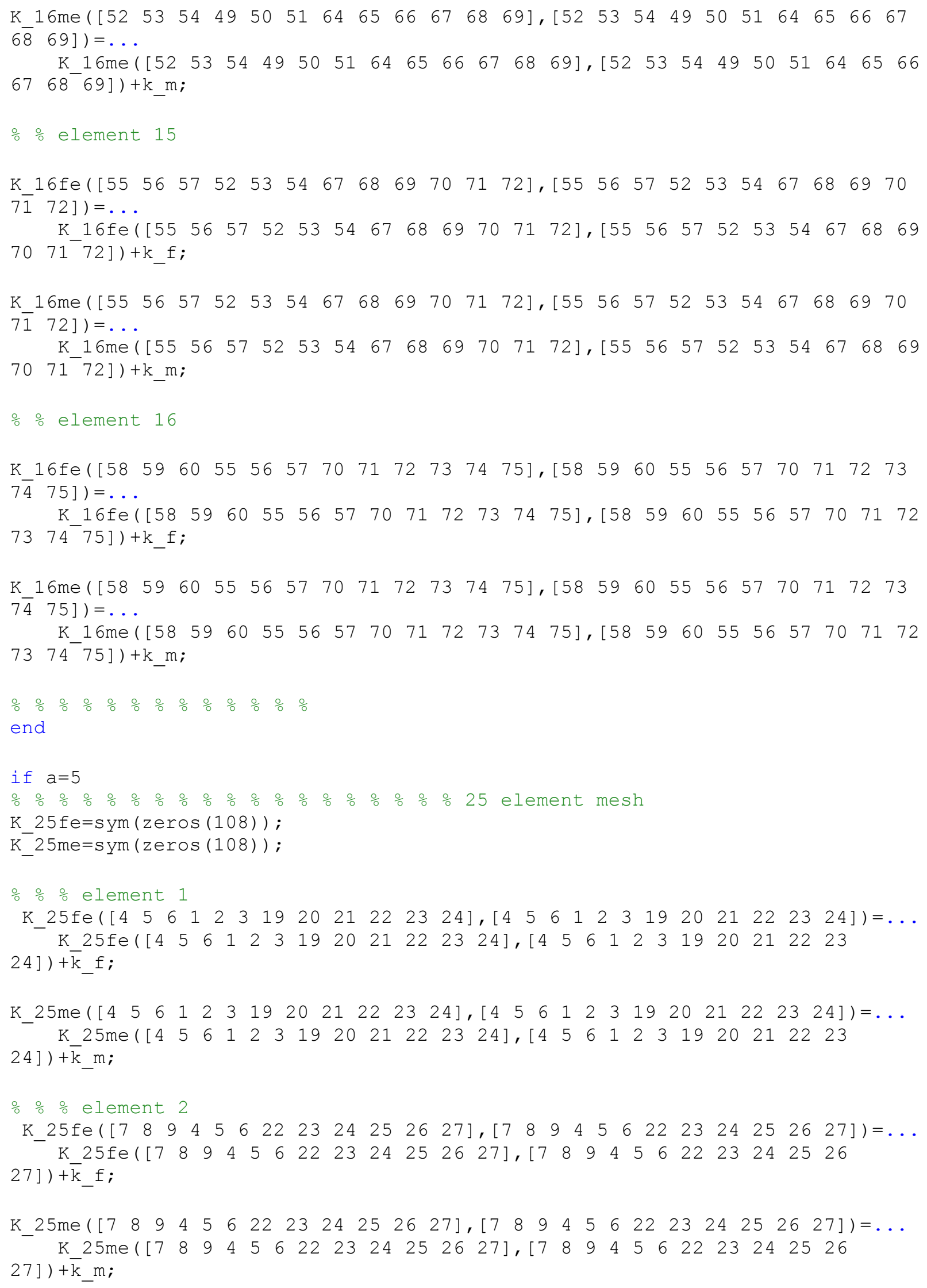




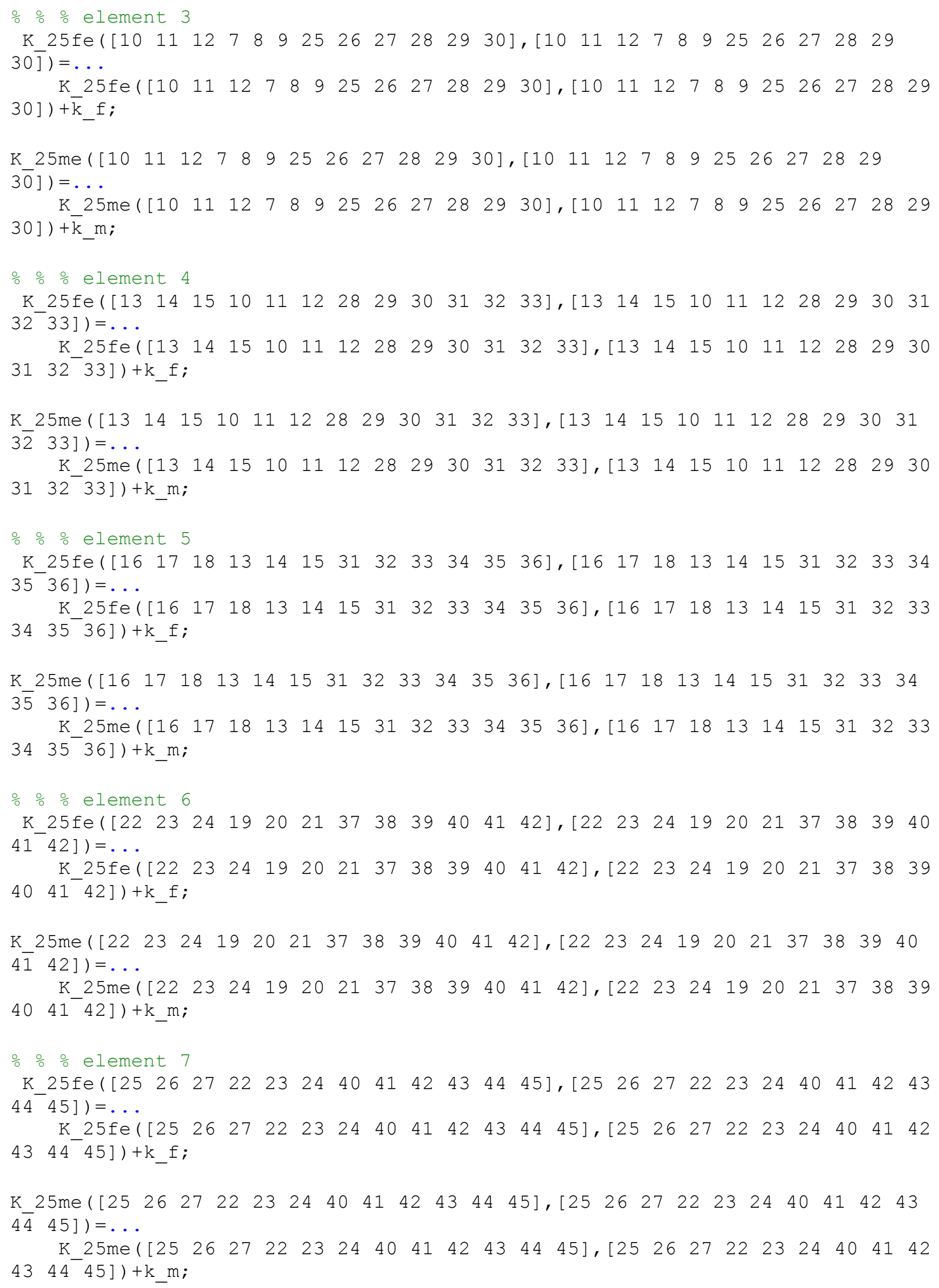




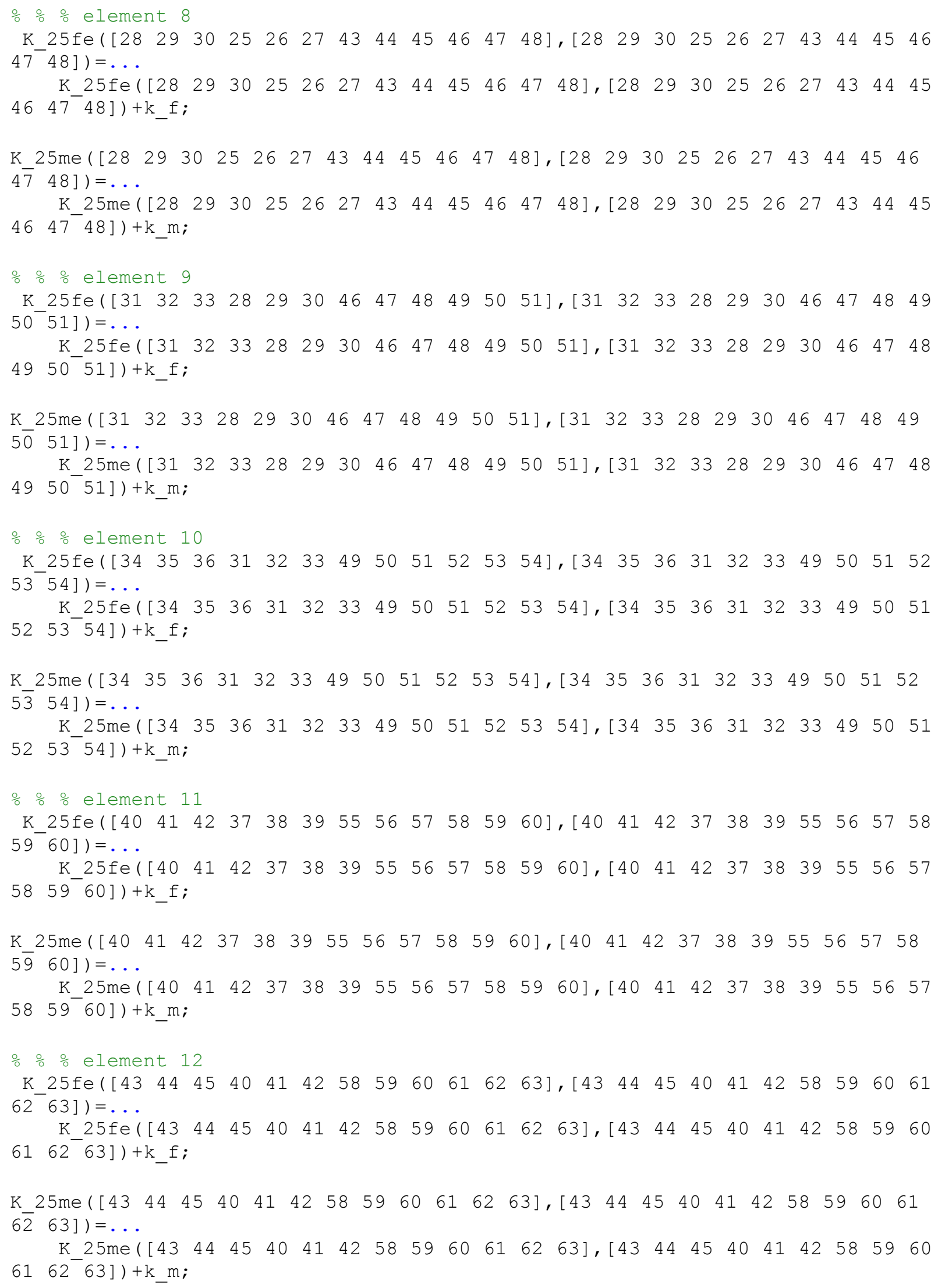




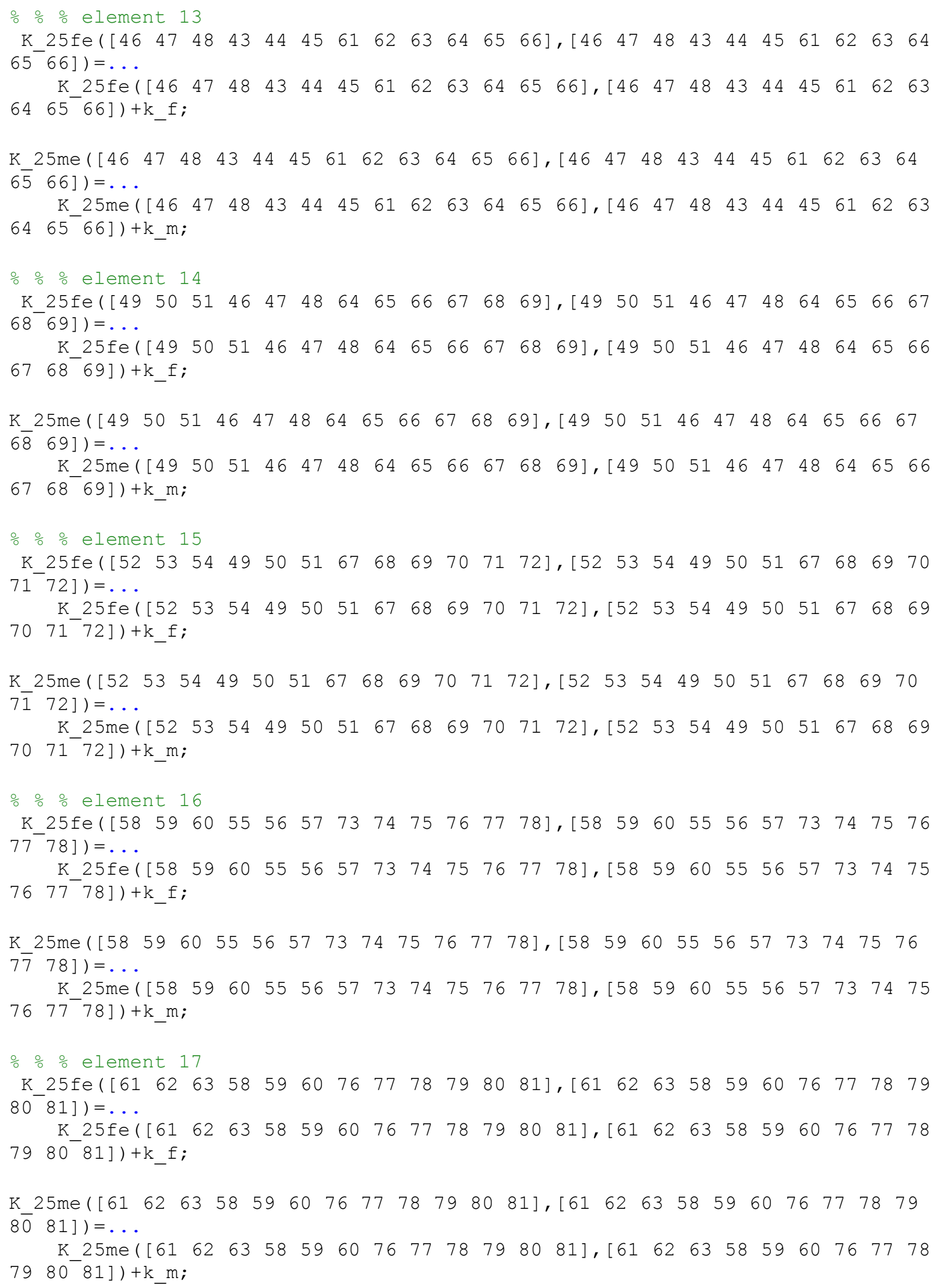




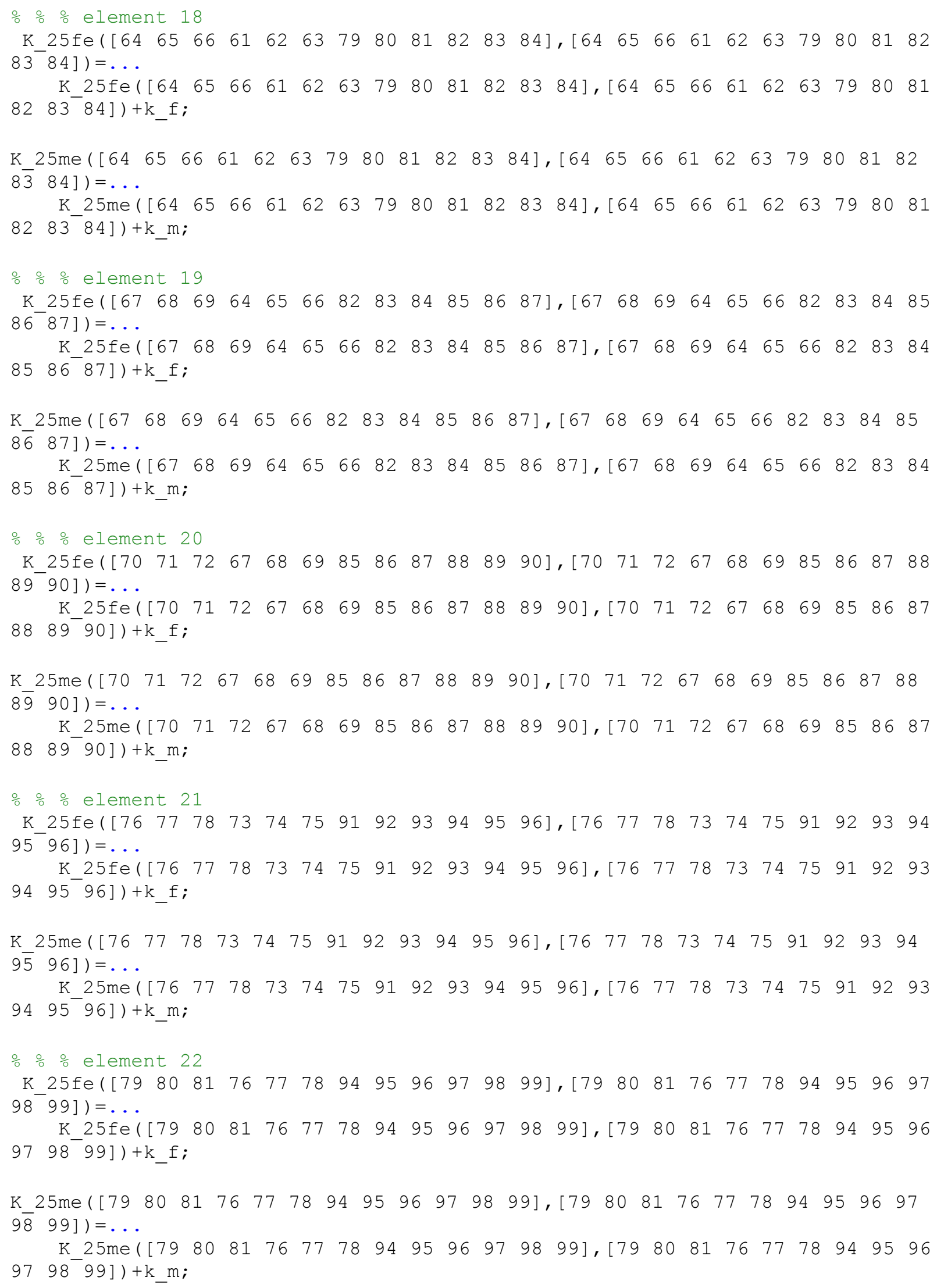




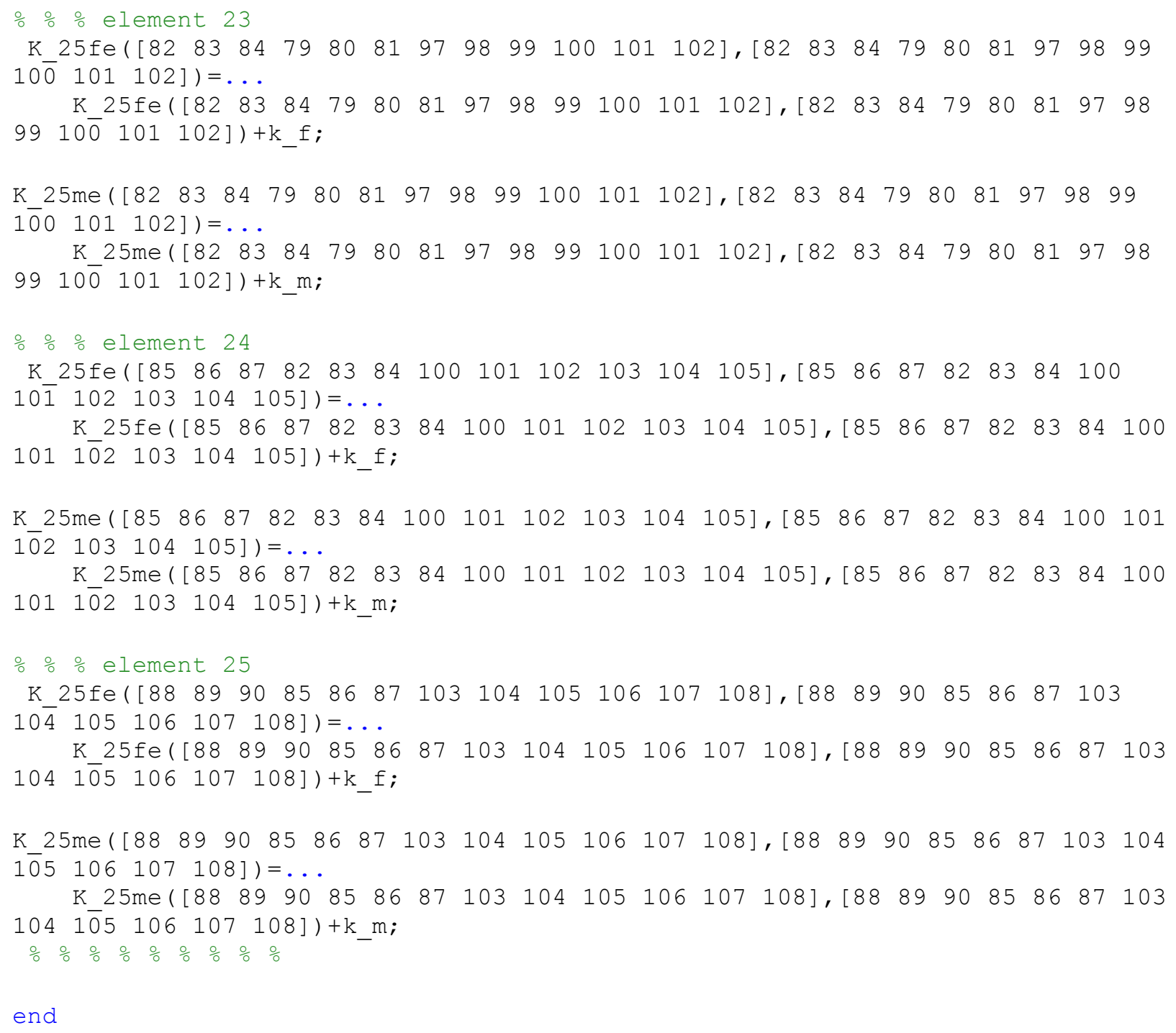




\section{References}

[1] E. Reissner, "The Effect of Transverse Shear Deformation on the Bending of Elastic Plates," Journal of Applied Mechanics, vol. 12, pp. 69-77, 1945.

[2] E. Reissner, "A Consistment Treatment of Transverse Shear Deformations in Laminated Anisotropic Plates," AIAA Journal, vol. 10, p. 716-718, 1972.

[3] R. D. Mindlin, "Influence of Rotatory Inertia and Shear on Flexural Motions of Isotropic Elastic Plates," Journal of Applied Mechanics, vol. 18, pp. 31-38, 1951.

[4] A. W. Leissa, "Vibration of Plates," National Aeronautics and Space Administration (NASA-SP-160), Washington, D.C., 1969.

[5] A. W. Leissa, "Recent Research in Plate Vibrations: Classical Theory," The Shock and Vibration Digest, vol. 9, no. 10, p. 13-24, 1977.

[6] A. W. Leissa, "Recent Research in Plate Vibrations: Complicating Effects," The Shock and Vibration Digest, vol. 9, no. 11, p. 21-35, 1977.

[7] A. W. Leissa, "Plate Vibration Research,1976-1980: Classical Theory," The Shock and Vibration Digest, vol. 13, no. 9, p. 11-22, 1981.

[8] A. W. Leissa, "Plate Vibration Research, 1976-1980: Complicating Effects," The Shock and Vibration Digest, vol. 13, no. 10, p. 19-36, 1981.

[9] A. W. Leissa, "Recent Research in Plate Vibrations, 1981-1985, Part II: Complicating Effects," The Shock and Vibration Digest, vol. 19, no. 3, p. 10-24, 1987.

[10] J. N. Reddy and N. D. Phan, "Stability and Vibration of Isotropic, Orthotropic and Laminated Plates according to a Higher Order Shear Deformation Theory," Journal of Sound and Vibration, vol. 98, pp. 157-170, 1985.

[11] N. R. Senthilnathan, S. P. Lim, K.H. Lee and S. Chow, "Vibration of Laminated Orthotropic Plates Using a Simplified Higher-Order Deformation Theory," Composite Structures, vol. 10, pp. 211-229, 1988.

[12] C. W. Bert and T. L. Chen, "Effect of Shear Deformation on Vibration of Antisymmetric Angle-Ply Laminated Rectangular Plates," International Journal of Solids and Structures, vol. 14, pp. 465-473, 1978.

[13] I. D. Kaplevatsky and V. O. Shestopal, "Bending and Buckling of Multilayer Thin Plates," Acta Mechanica, vol. 43, pp. 169-176, 1982.

[14] J.-L. Doong, T.-J. Chen and L.-W. Chen, "Vibration and Stability of an Initially Stressed Laminated Plate Based on a Higher Order Deformation Theory," Composite Structures, vol. 7, pp. 285-310, 
1987.

[15] C. W. Bert, "Optimal Design of a Composite-Material Plate to Maximize Its Fundamental Frequency," Journal of Sound and Vibration, vol. 50, pp. 229-23, 1977.

[16] M. Levy, "Sur L'equilibre elasticque d'une Plaque Rectangulaire," C.R. Acad. Science, vol. 129, pp. 535-539, 1899.

[17] John William Strutt, 3rd Baron Rayleigh, Theory of Sound (Volume 1), Macmillan, London; reprinted 1945 by Dover, New York, 1877.

[18] W. Ritz, "Uber Eine Neue Methode zur Losung Gewisser Variations Probleme der Mathematischen Physik.," Journal fur Reine und Angewandte Mathematik, vol. 135, p. 1-61, 1909.

[19] S. M. Dickinson and A. Di Blasio, "On the Use of Orthogonal Polynomials in the Rayleigh- Ritz Method for the Study of the Flexural Vibration and Buckling of Isotropic and Orthotropic Rectangular Plates," Journal of Sound and Vibration, vol. 108, pp. 51-62, 1986.

[20] D. J. Dawe and O. L. Roufaeil, "Rayleigh-Ritz Vibration Analysis of Mindlin Plates," Journal of Sound and Vibration, vol. 69, p. 345-359, 1980.

[21] O. L. Roufaeil and D. J. Dawe, "Rayleigh-Ritz Vibration Analysis of Rectangular Mindlin Plates Subjected to Membrane Stresses," Journal of Sound and Vibration, vol. 85, p. 263-275, 1982.

[22] L. W. Chen and J. L. Doong, "Large Amplitude Vibration of an Initially Stressed Moderately Thick Plate," Journal of Sound and Vibration, vol. 89, p. 499-508, 1983.

[23] B. Magrab, "Natural Frequencies of Elastically Supported Orthotropic Rectangular Plates," Journal of the Acoustical Society of America, vol. 61, p. 79-83, 1977.

[24] J. A. Bowlus, A. N. Palazotto and J. M. Whitney, "Vibration of Symmetrically Laminated Rectangular Plates Considering Deformation and Rotatory Inertia," AIAA Journal, vol. 25, pp. 1500-1511, 1987.

[25] K. S. Sivakumaran, "Natural Frequencies of Symmetrically Laminated Rectangular Plates with Free Edges," Composite Structures, vol. 7, p. 191-204, 1987.

[26] R. D. Henshell, D. Walters and G. B. Warburton, "A New Family of Curvilinear Plate Bending," Journal of Sound and Vibration, vol. 20, no. 3, pp. 381-397, 1972.

[27] J. N. Reddy, "Free vibration of Antisymmetric, Angle-ply Laminated Plates Including Transverse Shear Deformation by the Finite Element Method," Journal of Sound and Vibration, vol. 66, p. 565576, 1979.

[28] J. N. Reddy, "A Review of the Literature on Finite Element Modelling of Laminated Composite Plates and Shells," The Shock and Vibration Digest, vol. 17, no. 4, p. 3-8, 1985.

[29] C. W. Bert, J. N. Reddy, W. C. Chao and V. S. Reddy, "Vibration of Thick Rectangular Plates of Bimodulus Composite Materials," Journal of Applied Mechanics, American Society of Mechanical 
Engineers, vol. 48, no. 2, p. 371-376, 1981.

[30] J. N. Reddy, "Large Amplitude Flexural Vibration of Layered Composite Plates with Cut-Outs," Journal of Sound and Vibration, vol. 83, p. 1-10, 1982.

[31] J. N. Reddy and T. Kuppusamy, "Natural Vibration of laminated Anisotropic Plates," Journal of Sound and Vibration, vol. 94, p. 63-69, 1984.

[32] Y. V. K. S. Rao and G. Singh, "Vibration of Corner Supported Thick Composite Plates," Journal of Sound and Vibration, vol. 111, p. 510-514, 1986.

[33] C. N. Chang and F. K. Chiang, "Vibration Analysis of a Thick Plate With an Interior Cut-Out by a Finite Element Method," Journal of Sound and Vibration, vol. 125, p. 477-486, 1988.

[34] L. C. Shiau and J. T. Chang, "Transverse Shear Effect on Vibration of Laminated Plate Using HigherOrder Plate Element," Computers and Structures, vol. 39, p. 735-740, 1991.

[35] M. Ganapathi, T. K. Vardan and B. S. Sarma, "Nonlinear Flexural Vibrations of Laminated Orthotropic plates," Computers and Structures, vol. 39, p. 685-688, 1991.

[36] Y. K. Cheung and W. L. Kwok, "Dynamic analysis of Circular and Sector Thick, Layered Plates," Journal of Sound and Vibration, vol. 42, p. 147-158, 1975.

[37] L. W. Chen and C. C. Chen, "Asymmetric Vibration and Dynamic stability of Bimodulus thick Annular Plates," Computers and Structures, vol. 31, p. 1013-1022, 1989.

[38] W. H. Wittrick and F. W. Williams, "A General Algorithm for Computing Natural Frequencies of Elastic Structures," Quarterly Journal of Mechanics and Applied Mathematics, vol. 24, no. 3, p. 263284, 1971.

[39] J. R. Banerjee and F. W. Williams, "Coupled Bending-Torsional Dynamic Stiffness Matrix of an Axially Loaded Timoshenko Beam Element.," International Journal of Solids and Structures, vol. 31, pp. 749$762,1994$.

[40] J. R. Banerjee, "Dynamic Stiffness Formulation for Structural Elements: A General Approach," Computers \& Structures, vol. 63, no. 1, pp. 101-103, 1997.

[41] J. R. Banerjee, "Free Vibration of Sandwich Beams Using the Dynamic Stiffness Method," Computers \& Structures, vol. 81, pp. 1915-1922, 2003.

[42] J. R. Banerjee and A. J. Sobey, "Dynamic Stiffness Formulation and Free Vibration Analysis of A Three-Layered Sandwich Beam," International Journal of Solids and Structures, vol. 42, pp. 21812197, 2005.

[43] J. R. Banerjee, C. W. Cheung, R. Morishima, M. Perera and J. Njuguna, "Free Vibration of A Threelayered Sandwich Beam Using The Dynamic Stiffness Method and Experiment," International Journal of Solids and Structures, vol. 44, pp. 7543-7563, 2007. 
[44] J. R. Banerjee, H. Su and C. Jayatunga, "A Dynamic Stiffness Element for Free Vibration Analysis of Composite Beams and its application to Aircraft Wings," Computers and Structures, 2008.

[45] J. B. Casimira, S. Kevorkianb and T. Vinh, "The Dynamic Stiffness Matrix of Two-Dimensional Elements: Application to Kirchhoff's Plate Continuous Elements," Journal of Sound and Vibration, vol. 287, p. 571-589, 2005.

[46] S. M. Hashemi and J. M. Richard, "A DFE Method for Free Vibrations of Bending - Torsion Coupled Beams," Aerospace Science and Technology, vol. 4, p. 41 - 55, 2000.

[47] S. M. Hashemi and A. Roach, "A Dynamic Finite Element for the Free Vibration Analysis of Extension-Torsion Coupled Composite Beams," Mathematics in Engineering, Science and Aerospace (MESA), The Transdisciplinary International Journal, vol. 1, no. 3, p. $221-23,2010$.

[48] S. M. Hashemi and E. J. Adique, "A Quasi - Exact Dynamic Finite Element for Free Vibration Analysis of Sandwich Beams," Applied Composite Materials, vol. 17, p. 259-269, 2010.

[49] S. M. Hashemi and M. J. Richard, "Free Vibrational Analysis of Axially Loaded Bending - Torsion Coupled Beams: A Dynamic Finite Element," Computers and Structures, vol. 77, p. $711-724,2000$ ).

[50] T. Megson, Aircraft Structures for Engineering Students, Elsevier, 2012.

[51] A. Adini and R. W. Clough, "Analysis of Plate Bending by The Finite Element Method," A Report to the National Science Foundation, U.S.A., G. 7337, 1961.

[52] D. L. Logan, A First Course in the Finite Elemenet Method, Thomson, 2007.

[53] J. Dow, A Unified Approach to the Finite Element Method and Error Analysis Procedures, Elsevier.

[54] G. B. Thomas Jr. and R. L. Finney, Calculus, Pearson.

[55] K.-J. Bathe, Finite Element Procedures, Klaus-Jurgen Bathe, 2006.

[56] R. J. Melosh, "Structural Analysis of Solids," ASCE Structural Journal, vol. 89, no. 4, pp. 205-248, 1963. 